TRANSACTIONS OF THE

AMERICAN MATHEMATICAL SOCIETY

Volume 357, Number 12, Pages 4681-4717

S 0002-9947(05)03971-1

Article electronically published on June 29, 2005

\title{
QUANTUM GROUPS, DIFFERENTIAL CALCULI AND THE EIGENVALUES OF THE LAPLACIAN
}

\author{
J. KUSTERMANS, G. J. MURPHY, AND L. TUSET
}

\begin{abstract}
We study $*$-differential calculi over compact quantum groups in the sense of S.L. Woronowicz. Our principal results are the construction of a Hodge operator commuting with the Laplacian, the derivation of a corresponding Hodge decomposition of the calculus of forms, and, for Woronowicz' first calculus, the calculation of the eigenvalues of the Laplacian.
\end{abstract}

\section{INTRODUCTION}

Compact quantum groups and differential calculi over them were introduced by S.L. Woronowicz in a series of seminal papers [13, 14, 16. The theory of compact quantum groups is now well established - we refer to 8 and 11 for recent expositions. On the other hand, the theory of differential calculi over quantum groups is still in a very rudimentary state of development. A possible reason for this is that although it is an important part of non-commutative geometry, it does not fit into the framework of A. Connes. The principal difference from Connes' theory is that the appropriate "integrals" on quantum differential calculi are not graded traces but twisted graded traces. This involves the appearance of a twist automorphism under the integral sign and the transition from graded traces to twisted graded traces is analogous to that from ordinary traces in the theory of von Neumann algebras to KMS states. Some of the implications of this transition, such as the necessity of developing a twisted cyclic cohomology theory, are explored in [7].

In this paper we are principally concerned with studying the analogues of the classical operators of ordinary differential calculus over a manifold. Thus, we show that for the calculi that we study the differential admits an adjoint or co-differential and therefore one can define Dirac and Laplace operators. We also show that with suitable natural hypotheses on the calculi a Hodge operator exists. We then show that the calculus of forms admits a Hodge decomposition. This is surprising, since such a decomposition is known not to exist in general in the setting of noncommutative differential geometry.

We derive some general results on the eigenvalues of the Laplacian; specifically, we obtain formulas for the eigenvalues of the Laplacian when restricted to the spaces of lowest- and highest- dimensional forms. (The eigenvalues of the Dirac operator can be calculated from those of the Laplacian.)

Received by the editors January 23, 2001.

2000 Mathematics Subject Classification. Primary 58B32, 58B34.

The first author was supported by the National Science Foundation of Flanders.

(C)2005 American Mathematical Society 
The differential calculi that we are concerned with are assumed to be left invariant (this involves no loss of significant generality, since all the interesting examples in the theory are left invariant). The choice of a suitable inner product on the space of forms reduces to the question of choosing a suitable inner product on the space of invariant forms, which is finite dimensional in our theory. For many results this choice is quite arbitrary, since one needs only assume that the inner product is chosen so that forms of different dimensions are orthogonal. However, we believe that for the further development of the theory the choice of inner product and what kind of properties it should have will play a more significant role. We take up this question and show that one can always choose the inner product in such a way that the Hodge operator corresponding to this choice has such desirable properties as being a unitary that commutes with the Laplacian. We also show that the inner product can be chosen so that the twist automorphism corresponding to the "volume integral" on the calculus of forms is positive.

Connected with this, we show that under a natural condition on the twist automorphism, this twist automorphism can be seen as the generator of a one-parameter group of automorphisms on the space of differential forms. This one-parameter group mimics the role played by the modular groups of normal faithful semi-finite weights on von Neumann algebras. It also provides us with a symmetry group like the one discussed in [6, VII.12].

The important question of calculating all the eigenvalues of the Laplacian is undertaken in the case of a specific calculus, namely the three-dimensional calculus of Woronowicz over quantum $S U(2)$ that was the first ever example of a quantum calculus. The formulas for the eigenvalues are complicated, but we are nevertheless able to obtain bounds for them that enable us to show a "scaled boundedness" result for the commutator of the Dirac operator and an arbitrary element of the Hopf algebra underlying the quantum group. This shows that although this differential calculus does not fit into Connes' framework, it has properties that are close to those required to enable it to fit into the framework developed by A. Jaffe (this theory is an extension of Connes' theory). It may be that Jaffe's theory can, and should, be further developed to cover this example (and the quantum differential theory in general) - in any case, our example illustrates what is required to be done.

A very nice solution to similar kinds of problems has been developed in [9] and [10] by I. Heckenberger and A. Schüler. In these two papers, the authors look at metrics which they assume to satisfy the locality property but as a result the considered metrics cannot be positive in some cases (as in the case of Woronowicz' 4-dimensional differential calculi on quantum $S U(2))$. Nevertheless, a powerful theory emerges in their setting.

Commutator representations of differential calculi and the unboundedness of them are also intensively studied by K. Schmüdgen; see for instance [3] and [4].

Now we summarise what we do in each section of the paper: Section 2 introduces the basic concepts and terminology that will be needed throughout the paper and shows the existence of the co-differential under suitable hypotheses (Theorem 2.4). Section 3 is concerned with the existence of the Hodge operator (Theorem 3.2) and in Section 4 a Hodge decomposition is obtained (Theorem 4.1). In Section 5 we prove some general results on diagonalising the Laplacian on the forms of lowest and highest degree. Sections 6 and 7 are concerned with choosing the inner product on the invariant forms in such a way that the Hodge operator and the twist 
operator have desirable properties (Theorems 6.1 and 7.9). Section 8 is devoted to the calculation and analysis of the eigenvalues of the Laplacian for the threedimensional calculus of Woronowicz mentioned above. At the end of the paper we have included a brief appendix on some non-standard results on exponential one-parameter automorphism groups that we need.

\section{Differential CALCUli}

We begin by recalling some basic definitions.

If $\Omega$ is an algebra, a positive algebra grading on $\Omega$ is a sequence $\left(\Omega_{n}\right)_{n}$ of subspaces of $\Omega$ for which $\Omega=\bigoplus_{n=0}^{\infty} \Omega_{n}$ and $\Omega_{m} \Omega_{n} \subseteq \Omega_{n+m}$, for all $n, m \geq 0$. The pair $\left(\Omega,\left(\Omega_{n}\right)_{n}\right)$ is called a graded algebra.

A conjugate-linear map $\omega \mapsto \omega^{*}$ of degree zero is called a graded involution on $\Omega$ if $\left(\omega^{*}\right)^{*}=\omega$ and $\left(\omega_{1} \omega_{2}\right)^{*}=(-1)^{k l} \omega_{2}^{*} \omega_{1}^{*}$, for all $\omega \in \Omega$ and all pairs $\omega_{1}, \omega_{2} \in \Omega$ of degrees $k$ and $l$, respectively. The pair consisting of $\Omega$ together with this involution is called a graded $*$-algebra.

A graded $*$-derivation on $\Omega$ is a linear map $d: \Omega \rightarrow \Omega$ for which $d\left(\omega^{*}\right)=(d \omega)^{*}$ and $d\left(\omega^{\prime} \omega\right)=d\left(\omega^{\prime}\right) \omega+(-1)^{k} \omega^{\prime} d \omega$, for all $\omega^{\prime} \in \Omega_{k}$ and all $\omega \in \Omega$.

A graded $*$-differential algebra is a pair $(\Omega, d)$, where $\Omega$ is a graded $*$-algebra, $d$ is a graded $*$-derivation on $\Omega$ of degree 1 (as a linear map) and $d^{2}=0$. The elements of $\Omega$ are referred to as the forms of $(\Omega, d)$ and the elements of $\Omega_{k}$ as the $k$-forms. The operator $d$ is referred to as the differential.

Suppose now that $(\mathcal{A}, \Delta)$ is a Hopf $*$-algebra. (We shall usually refer to the algebra by the symbol $\mathcal{A}$ and suppress explicit reference to the co-multiplication $\Delta$. For Hopf algebra theory we refer the reader to [1.) We say that a graded $*$-differential algebra $(\Omega, d)$ is a $*$-differential calculus over $\mathcal{A}$ if the following conditions hold:

1. $\Omega_{0}=\mathcal{A}$ (as $*$-algebras) and, for all $k>0, \Omega_{k}$ is the linear span of all products $a_{0} d a_{1} \cdots d a_{k}$, where $a_{0}, \ldots, a_{k} \in \mathcal{A}$.

2. There exists an algebra homomorphism $\Delta_{\Omega}: \Omega \rightarrow \mathcal{A} \otimes \Omega$ such that $\Delta_{\Omega}(a)$ $=\Delta(a)$, for all $a \in \mathcal{A}$, and such that $\left(\operatorname{id}_{\mathcal{A}} \otimes d\right) \Delta_{\Omega}=\Delta_{\Omega} d$.

3. $d 1=0$.

Note that Condition 2 is called left covariance of the differential calculus. (We shall only be interested in left-covariant differential calculi.)

We now make a sequence of useful observations that follow from this definition:

Conditions 1 and 3 easily imply that the unit 1 of $\mathcal{A}$ is a unit for the algebra $\Omega$.

Since $d(a b)=(d a) b+a d b$, for $a, b \in \mathcal{A}$, it follows from Condition 1 that $\Omega_{1}$ is the linear span of all products $\left(d a_{1}\right) a_{0}$, where $a_{0}, a_{1} \in \mathcal{A}$. A simple inductive argument now shows that $\Omega_{k}$ is the linear span of all products $\left(d a_{k}\right) \cdots\left(d a_{1}\right) a_{0}$, where $a_{0}, \ldots, a_{k} \in \mathcal{A}$.

It is easily seen that the map $\Delta_{\Omega}$ is automatically of degree zero and $*$-preserving; that is, $\Delta_{\Omega}\left(\omega^{*}\right)=\Delta_{\Omega}(\omega)^{*}$.

Note that $\left(\Delta \otimes \operatorname{id}_{\Omega}\right) \Delta_{\Omega}=\left(\operatorname{id}_{\mathcal{A}} \otimes \Delta_{\Omega}\right) \Delta_{\Omega}$ and $\left(\varepsilon \otimes \operatorname{id}_{\Omega}\right) \Delta_{\Omega}=\operatorname{id}_{\Omega}$, where $\varepsilon$ is the co-unit of $\mathcal{A}$; hence, the map $\Delta_{\Omega}$ is a left co-action of the Hopf algebra $\mathcal{A}$ on $\Omega$. For the proof, see [7, Lemma 4.2].

Recall that a bi-module $\Gamma$ over $\mathcal{A}$ is said to be left covariant if there is a linear map $\Delta_{\Gamma}: \Gamma \rightarrow \mathcal{A} \otimes \Gamma$ such that $\left(\Delta \otimes \operatorname{id}_{\Gamma}\right) \Delta_{\Gamma}=\left(\operatorname{id}_{\mathcal{A}} \otimes \Delta_{\Gamma}\right) \Delta_{\Gamma}$ and $\left(\varepsilon \otimes \operatorname{id}_{\Gamma}\right) \Delta_{\Gamma}=$ $\operatorname{id}_{\Gamma}$, where $\varepsilon$ is the co-unit of $\mathcal{A}$ (that is, $\Delta_{\Gamma}$ is a left co-action) and $\Delta_{\Gamma}(a \gamma b)=$ $\Delta(a) \Delta_{\Gamma}(\gamma) \Delta(b)$, for all $\gamma \in \Gamma$ and $a, b \in \mathcal{A}$. An element $\gamma \in \Gamma$ is said to be left 
invariant if $\Delta_{\Gamma}(\gamma)=1 \otimes \gamma$. We denote by $\Gamma^{\text {inv }}$ the linear space of left-invariant elements of $\Gamma$.

We shall make use of the following results from the theory of left-covariant bimodules [5] (we do not state these results in their strongest forms). First, we need some notation. If $\chi$ is a linear functional on a Hopf algebra $\mathcal{A}$ and $a \in \mathcal{A}$, we write $\chi * a$ for $(\operatorname{id} \otimes \chi) \Delta(a)$ and $a * \chi$ for $(\chi \otimes \mathrm{id}) \Delta(a)$.

Theorem 2.1. Let $\Gamma$ be a left-covariant bi-module over a Hopf $*$-algebra $\mathcal{A}$. Then there is a unique isomorphism of left $\mathcal{A}$-modules from $\mathcal{A} \otimes \Gamma^{\mathrm{inv}}$ onto $\Gamma$ that maps $a \otimes \gamma$ onto $a \gamma$, for all $a \in \mathcal{A}$ and $\gamma \in \Gamma^{\text {inv }}$.

Theorem 2.2. Let $\mathcal{A}$ be a Hopf *-algebra with co-inverse $\kappa$. Let $\Gamma$ be a leftcovariant bi-module over $\mathcal{A}$ and suppose that $\gamma_{0}, \ldots, \gamma_{n}$ is a linear basis for $\Gamma^{\mathrm{inv}}$. Then $\gamma_{0}, \ldots, \gamma_{n}$ is a free left $\mathcal{A}$-module basis for $\Gamma$ and also a free right $\mathcal{A}$-module basis of $\Gamma$. Moreover, there exist linear functionals $f_{j k}$ on $\mathcal{A}$, for $j, k=0, \ldots, n$, such that $f_{j k}(a b)=\sum_{r=0}^{n} f_{j r}(a) f_{r k}(b)$ and $f_{j k}(1)=\delta_{j k}$ and for which we have the equations $\gamma_{j} a=\sum_{k=0}^{n}\left(f_{j k} * a\right) \gamma_{k}$ and $a \gamma_{j}=\sum_{k=0}^{n} \gamma_{k}\left(\left(f_{j k} \kappa^{-1}\right) * a\right)$.

If $(\Omega, d)$ is a $*$-differential calculus over a Hopf $*$-algebra $\mathcal{A}$, then it is clear from our earlier observations that $\Omega$ is a left-covariant bi-module over $\mathcal{A}$, so that Theorem 2.1 applies. We shall identify $\mathcal{A} \otimes \Omega^{\text {inv }}$ and $\Omega$, as left $\mathcal{A}$-modules, using the isomorphism of the theorem. Observe that each space $\Omega_{k}$ is also a left-covariant bi-module over $\mathcal{A}$.

It is clear that $\Omega^{\text {inv }}$ is not only a linear subspace of $\Omega$, but also a subalgebra closed under the involution $\omega \mapsto \omega^{*}$. For $k \geq 0$, set $\Omega_{k}^{\text {inv }}=\Omega_{k} \cap \Omega^{\text {inv }}$. It is easily seen that $\Omega^{\text {inv }}=\bigoplus_{k=0}^{\infty} \Omega_{k}^{\text {inv }}$, so that we have an induced algebra grading on $\Omega^{\text {inv }}$; hence, $\Omega^{\text {inv }}$ is a graded $*$-algebra. Since $\Omega^{\text {inv }}$ is clearly invariant under $d,\left(\Omega^{\text {inv }}, \partial\right)$ is a graded $*$-differential algebra, where $\partial$ is the restriction of $d$ to $\Omega^{\text {inv }}$.

Suppose now that $\mathcal{A}$ admits a Haar integral $h$; that is, $h$ is a unital linear functional on $\mathcal{A}$ for which (id $\otimes h) \Delta(a)=(h \otimes \mathrm{id}) \Delta(a)=h(a) 1$, for all $a \in \mathcal{A}$, where 1 is the unit of $\mathcal{A}$. We suppose further that $h$ is positive; that is, $h\left(a^{*} a\right) \geq 0$, for all $a \in \mathcal{A}$. It is well known $h$ is unique and automatically faithful; that is, if $h\left(a^{*} a\right)=0$, then $a=0$. We endow $\mathcal{A}$ with an inner product derived from $h$, by setting $(a \mid b)=h\left(b^{*} a\right)$, for $a, b \in \mathcal{A}$.

Now suppose that $(\Omega, d)$ is strongly finite dimensional; by this we mean that $\Omega^{\text {inv }}$ is finite dimensional (in this case Theorem 2.2 applies to $\Omega$ ). It follows that, for some integer $N, \Omega_{k}=0$ for $k>N$ and $\Omega_{N} \neq 0$. Hence, $\Omega$ is finite dimensional in the sense of [7]. The integer $N$ is the dimension of $(\Omega, d)$.

Choose an arbitrary inner product on $\Omega^{\text {inv }}$ for which the subspaces $\Omega_{k}^{\text {inv }}$ are pairwise orthogonal. Then endow $\Omega=\mathcal{A} \otimes \Omega^{\text {inv }}$ with the tensor product inner product. Since $\Omega_{k}=\mathcal{A} \otimes \Omega_{k}^{\text {inv }}$, the spaces $\Omega_{k}$ are pairwise orthogonal. We call an inner product on $\Omega$ graded if it has this property. We call an inner product left-invariant if $(a \omega \mid b \eta)=h\left(b^{*} a\right)(\omega \mid \eta)$, for all $a, b \in \mathcal{A}$ and $\omega, \eta \in \Omega^{\text {inv }}$.

Suppose that $\operatorname{dim}\left(\Omega_{N}^{\text {inv }}\right)=1$. Then it is easily seen that there is a unit vector $\theta$ of $\Omega_{N}^{\text {inv }}$ such that $\theta^{*}=\theta$ and that $\theta$ is unique up to sign. We shall call $\theta$ and $-\theta$ the volume elements of $(\Omega, d)$.

We shall now derive a very useful formula for the differential $d$. First we need to introduce some linear operators. If $\chi$ is a linear functional on $\mathcal{A}$, we denote by $E_{\chi}$ the linear operator on $\mathcal{A}$ defined by setting $E_{\chi}(a)=\chi * a$. If $\omega \in \Omega_{1}^{\text {inv }}$, we denote by $M_{\omega}$ the linear operator on $\Omega^{\text {inv }}$ defined by setting $M_{\omega}(\eta)=\omega \eta$. 
For the next theorem, let us recall the definition of the adjoint $\chi^{*}$ of $\chi$ : It is given by the formula $\chi^{*}(a)=\overline{\chi\left(\kappa(a)^{*}\right)}$, for all $a \in \mathcal{A}$. We shall also need to define $\bar{\chi}$ in $\mathcal{A}^{*}$ by $\bar{\chi}(a)=\chi\left(a^{*}\right)^{-}$.

If $T$ is an operator on an inner product space $X$, we shall denote by $T^{*}$ the unique operator - if it exists - for which $(T(x) \mid y)=\left(x \mid T^{*}(y)\right)$, for all $x, y \in X$. In the case $T^{*}$ exists, we say $T$ is adjointable and call $T^{*}$ the adjoint of $T$. (Of course, if $X$ is a Hilbert space and $T$ a bounded linear operator on $X, T^{*}$ necessarily exists. The problem of existence arises with unbounded operators and incomplete spaces.) If $T$ is adjointable and $T=T^{*}$, then the eigenvalues of $T$ are real. Similarly, the eigenvalues of $T T^{*}, T^{*} T$ and $T^{*} T+T T^{*}$ are non-negative. (The usual trivial proofs apply.)

Lemma 2.3. Let $\mathcal{A}$ be a Hopf *-algebra admitting a Haar integral $h$ and let $\chi$ be a linear functional on $\mathcal{A}$. Then $E_{\chi}$ is adjointable and $E_{\chi}^{*}=E_{\chi^{*}}$.

Proof. Let $a, b \in \mathcal{A}$. Using the equality

$$
\kappa((h \otimes \mathrm{id})((q \otimes 1) \Delta(p)))=(h \otimes \mathrm{id})(\Delta(q)(p \otimes 1))
$$

(see the proof of Proposition 3.11 of [12]), we see that

$$
\begin{aligned}
\left(E_{\chi}(a) \mid b\right) & =h\left(b^{*}(\mathrm{id} \otimes \chi) \Delta(a)\right)=\chi\left((h \otimes \mathrm{id})\left(\left(b^{*} \otimes 1\right) \Delta(a)\right)\right) \\
& =\chi\left(\kappa^{-1}\left((h \otimes \mathrm{id})\left(\Delta\left(b^{*}\right)(a \otimes 1)\right)\right)\right)=h\left(\left(\mathrm{id} \otimes \chi \kappa^{-1}\right) \Delta\left(b^{*}\right) a\right) \\
& =h\left(\left(\left(\operatorname{id} \otimes \chi^{*}\right) \Delta(b)\right)^{*} a\right)=h\left(E_{\chi^{*}}(b)^{*} a\right)=\left(a \mid E_{\chi^{*}}(b)\right) .
\end{aligned}
$$

Hence, $E_{\chi}$ has adjoint $E_{\chi^{*}}$, as required.

If $\omega_{1}, \ldots, \omega_{M}$ is an orthonormal basis for $\Omega_{1}^{\text {inv }}$, there exist unique linear functionals $\chi_{1}, \ldots, \chi_{M}$ on $\mathcal{A}$ such that

$$
d a=\sum_{r=1}^{M}\left(\chi_{r} * a\right) \omega_{r} .
$$

(See [5].)

Theorem 2.4. Let $(\Omega, d)$ be a strongly finite-dimensional *-differential calculus over a Hopf *-algebra $\mathcal{A}$ admitting a Haar integral. Then $d$ is adjointable and $d^{*}$ is of degree -1 . Indeed, if $\omega_{1}, \ldots, \omega_{M}$ and $\chi_{1}, \ldots, \chi_{M}$ are as in Equation (1), then

$$
d=\operatorname{id}_{\mathcal{A}} \otimes \partial+\sum_{j=1}^{M} E_{\chi_{j}} \otimes M_{\omega_{j}}
$$

and

$$
d^{*}=\operatorname{id}_{\mathcal{A}} \otimes \partial^{*}+\sum_{j=1}^{M} E_{\chi_{j}^{*}} \otimes M_{\omega_{j}}^{*}
$$

Proof. Recall that we identify $\Omega$ and $\mathcal{A} \otimes \Omega^{\text {inv }}$ by identifying $a \omega$ with $a \otimes \omega$, for $a \in$ $\mathcal{A}$ and $\omega \in \Omega^{\text {inv }}$. Since $d(a \omega)=(d a) \omega+a d \omega=\sum_{j=1}^{M}\left(\chi_{j} * a\right) \omega_{j} \omega+a d \omega$, we have $d=$ $\operatorname{id}_{\mathcal{A}} \otimes \partial+\sum_{j=1}^{M} E_{\chi_{j}} \otimes M_{\omega_{j}}$. Adjointability of $d$ now follows from adjointability of the operators $E_{\chi_{j}}$, and $M_{\omega_{j}}$ and $\partial$. The operators $E_{\chi_{j}}$ are adjointable by Lemma 2.3 , and the other operators are adjointable since $\Omega^{\text {inv }}$ is finite dimensional. The formula for $d^{*}$ in the statement of the theorem follows immediately. Since $d$ is of degree +1 , it is trivially verified that $d^{*}$ is of degree -1 . 
The operator $d^{*}$ is called the co-differential of $d$. The sum $D=d+d^{*}$ is the Dirac operator and the square $\nabla=\left(d+d^{*}\right)^{2}$ the Laplacian. Since $d^{2}=d^{* 2}=0$, we have $\nabla=d d^{*}+d^{*} d$. We call the forms $\omega \in \Omega$ such that $\nabla(\omega)=0$ the harmonic forms of $(\Omega, d)$ and denote the linear space of these forms by $\Omega_{\nabla}$.

\section{The Hodge Operator}

To avoid tedious repetition of hypothesis, throughout this section we denote by $(\Omega, d)$ a strongly finite-dimensional $*$-differential calculus of dimension $N$ over a Hopf *-algebra $\mathcal{A}$ admitting a Haar integral $h$. We suppose that $\operatorname{dim}\left(\Omega_{N}^{\mathrm{inv}}\right)=1$ and we denote by $\theta$ a volume element of $(\Omega, d)$. We also fix a graded, left invariant inner product $(. \mid$.$) on \Omega$.

We define a linear functional $\int$ on $\Omega$ by setting $\int \omega=0$, if $\omega$ is a $k$-form for which $k<N$, and $\int \omega=h(a)$, if $\omega$ is an $N$-form for which $\omega=a \theta$, where $a \in \mathcal{A}$. We call $\int$ the integral on $\Omega$ associated to the volume element $\theta$.

We say that $(\Omega, d)$ is non-degenerate if, whenever $0 \leq k \leq N$ and $\omega \in \Omega_{k}$, and $\omega^{\prime} \omega=0$, for all $\omega^{\prime} \in \Omega_{N-k}$, then necessarily $\omega=0$.

Lemma 3.1. If $(\Omega, d)$ is non-degenerate, and $\eta$ is an element of $\Omega$ for which $\int \omega \eta=0$, for all $\omega \in \Omega$, then $\eta=0$.

Proof. We may clearly reduce to the case where $\eta$ is a $k$-form, for some $k \leq N$. Then, if $\omega$ belongs to $\Omega_{N-k}$, we have $\omega \eta=a \theta$, for some element $a \in \mathcal{A}$, since $\operatorname{dim}\left(\Omega_{N}^{\mathrm{inv}}\right)=1$. Hence, $\int a^{*} \omega \eta=\int a^{*} a \theta=h\left(a^{*} a\right)=0$ and therefore, by faithfulness of $h, a=0$. Hence, $\omega \eta=0$, for all forms $\omega$ in $\Omega_{N-k}$. Therefore, by non-degeneracy of $\Omega, \eta=0$.

The property enjoyed by $\int$ in this lemma is called (left) faithfulness [7].

Theorem 3.2. Suppose $(\Omega, d)$ is non-degenerate. Then there exists a unique left $\mathcal{A}$-linear operator $L$ on $\Omega$ such that $L\left(\Omega_{k}\right)=\Omega_{N-k}$ for $k=0, \ldots, N$ and such that $\int \omega^{*} L\left(\omega^{\prime}\right)=\left(\omega^{\prime} \mid \omega\right)$, for all $\omega, \omega^{\prime} \in \Omega$. Moreover, $L$ is bijective.

Proof. Uniqueness of $L$ follows immediately from Lemma 3.1, so we confine ourselves to showing existence. Let $\eta \in \Omega_{k}^{\text {inv }}$ and $\xi \in \Omega_{l}^{\text {inv }}$, where $0 \leq k, l \leq N$. If $k+l=N$, then $\eta^{*} \xi=\gamma(\xi, \eta) \theta$, for a unique scalar $\gamma(\xi, \eta)$. Extend $\gamma$ to a sesquilinear function on $\Omega^{\text {inv }}$ by setting $\gamma(\xi, \eta)=0$ if $k+l \neq N$. Let $F$ be the unique linear operator on $\Omega^{\text {inv }}$ for which $(F(\eta) \mid \xi)=\gamma(\eta, \xi)$, for all $\eta, \xi \in \Omega^{\text {inv }}$. It is easily checked that $F\left(\Omega_{k}^{\text {inv }}\right) \subseteq \Omega_{N-k}^{\text {inv }}$ and that, since $\Omega$ is non-degenerate, $F$ is injective. Since $\Omega^{\text {inv }}$ is finite dimensional, $F$ is therefore bijective. It follows that $F\left(\Omega_{k}^{\text {inv }}\right)=\Omega_{N-k}^{\text {inv }}$. Now set $L=\mathrm{id} \otimes F^{-1}$, where we identify $\Omega$ with $\mathcal{A} \otimes \Omega^{\text {inv }}$, as usual. It is clear that $L$ is a bijective left $\mathcal{A}$-linear operator on $\Omega$ and that $L\left(\Omega_{k}\right)=\Omega_{N-k}$, for $k=0, \ldots, N$.

To show that $\int \omega^{*} L\left(\omega^{\prime}\right)=\left(\omega^{\prime} \mid \omega\right)$, for all $\omega, \omega^{\prime} \in \Omega$, we may clearly suppose that $\omega$ and $\omega^{\prime}$ are both $r$-forms, for some $r=0, \ldots, N$, using the fact that both sides of the equation are graded sesquilinear forms. Since $\Omega_{r}$ is a left-covariant bi-module, and $\Omega_{r}^{\text {inv }}$ is finite dimensional, we may apply Theorem 2.2 to choose a basis $\theta_{1}, \ldots, \theta_{M}$ for $\Omega_{r}^{\text {inv }}$ and associated linear functionals $g_{j k}$ on $\mathcal{A}$, where $j, k=1, \ldots, M$, such that $a \theta_{j}=\sum_{k=1}^{M} \theta_{k}\left(g_{j k} * a\right)$, for all $a \in \mathcal{A}$. We may also suppose that $g_{j k}(1)=\delta_{j k}$. To prove $\int \omega^{*} L\left(\omega^{\prime}\right)=\left(\omega^{\prime} \mid \omega\right)$ we may make an additional simplification and suppose now that $\omega=a \theta_{j}$ and $\omega^{\prime}=b \theta_{l}$, for some $a, b \in \mathcal{A}$ 
and some indices $j$ and $l$ in the set $\{1, \ldots, M\}$. Then

$$
\begin{aligned}
& \int \omega^{*} L\left(\omega^{\prime}\right)=\int \theta_{j}^{*} a^{*} b F^{-1}\left(\theta_{l}\right)=\int\left(b^{*} a \theta_{j}\right)^{*} F^{-1}\left(\theta_{l}\right) \\
& =\int \sum_{k=1}^{M}\left(\theta_{k}\left(g_{j k} *\left(b^{*} a\right)\right)\right)^{*} F^{-1}\left(\theta_{l}\right)=\int \sum_{k=1}^{M}\left(g_{j k} *\left(b^{*} a\right)\right)^{*} \theta_{k}^{*} F^{-1}\left(\theta_{l}\right) \\
& =\sum_{k=1}^{M} \int\left(\bar{g}_{j k} *\left(a^{*} b\right)\right) \gamma\left(F^{-1}\left(\theta_{l}\right), \theta_{k}\right) \theta=\sum_{k=1}^{M} \int\left(\bar{g}_{j k} *\left(a^{*} b\right)\right)\left(\theta_{l} \mid \theta_{k}\right) \theta \\
& =\sum_{k=1}^{M} h\left(\bar{g}_{j k} *\left(a^{*} b\right)\right)\left(\theta_{l} \mid \theta_{k}\right)=\sum_{k=1}^{M} h\left(a^{*} b\right) \bar{g}_{j k}(1)\left(\theta_{l} \mid \theta_{k}\right) \\
& =h\left(a^{*} b\right)\left(\theta_{l} \mid \theta_{j}\right)=\left(b \theta_{l} \mid a \theta_{j}\right)=\left(\omega^{\prime} \mid \omega\right) .
\end{aligned}
$$

This completes the proof.

The operator $L$ whose existence is shown in Theorem 3.2 is called the Hodge operator on $\Omega$. Let us observe that, since $L\left(\Omega_{k}^{\text {inv }}\right)=\Omega_{N-k}^{\text {inv }}$, for $0 \leq k \leq N$, we have $\operatorname{dim}\left(\Omega_{k}^{\mathrm{inv}}\right)=\operatorname{dim}\left(\Omega_{N-k}^{\mathrm{inv}}\right)$.

Observe also that $L(1)=\theta$ and $L(\theta)=1$ (these equalities are easily verified from the definition of $F$ in the proof of the theorem).

The following result gives a formula for the co-differential that is familiar from classical differential geometry.

Theorem 3.3. Suppose $(\Omega, d)$ is non-degenerate and $d\left(\Omega_{N-1}^{\text {inv }}\right)=0$. Then, if $0 \leq$ $k \leq N$ and $\omega$ is a $k$-form of $\Omega$, we have

$$
d^{*} \omega=(-1)^{k} L^{-1} d L(\omega) .
$$

Proof. If $k=0$, then $d^{*}(\omega)=0$, since $d^{*}$ is of degree -1 . Also, $d L(\omega)=0$, since $L(\omega)$ is in $\Omega_{N}$ and $d\left(\Omega_{N}\right)=0$. Hence, $d^{*} \omega=(-1)^{k} L^{-1} d L(\omega)$ in this case.

Suppose now that $k>0$. Clearly, $L^{-1} d L(\omega) \in \Omega_{k-1}$. Hence, if $\omega^{\prime} \in \Omega_{k-1}$, then $\left((-1)^{k} L^{-1} d L(\omega) \mid \omega^{\prime}\right)=(-1)^{k} \int \omega^{\prime} d L(\omega)=\int d\left(\omega^{\prime}\right)^{*} L(\omega)=\left(\omega \mid d\left(\omega^{\prime}\right)\right)=$ $\left(d^{*} \omega \mid \omega^{\prime}\right)$. Here we have used the fact that $\int$ is closed; that is, $\int d=0$. This is a consequence of our assumption that $d\left(\Omega_{N-1}^{\text {inv }}\right)=0$ [7, Corollary 4.9]. Therefore, $d^{*} \omega=(-1)^{k} L^{-1} d L(\omega)$ in this case also.

\section{The Hodge DeComposition}

Suppose that $G=(A, \Delta)$ is a compact quantum group in the sense of Woronowicz [11, 14. Let $\hat{G}$ denote the set of equivalence classes of irreducible unitary representations of $G$. For each $\alpha \in \hat{G}$, choose once and for all an irreducible unitary representation $U^{\alpha}=\left(U_{j k}^{\alpha}\right) \in M_{N_{\alpha}}(\mathbf{C}) \otimes A$ belonging to $\alpha$. Of course, $N_{\alpha}$ is the dimension of $U^{\alpha}$. Also, $\Delta\left(U_{j k}^{\alpha}\right)=\sum_{r=1}^{N_{\alpha}} U_{j r}^{\alpha} \otimes U_{r k}^{\alpha}$, for $j, k=1, \ldots, N_{\alpha}$.

Recall that the linear $\operatorname{span} \mathcal{A}=\mathcal{A}_{G}$ of the set $\left\{U_{j k}^{\alpha} \mid \alpha \in \hat{G}, j, k=1, \ldots, N_{\alpha}\right\}$ is a Hopf $*$-algebra, when endowed with the restriction of the co-multiplication $\Delta$ on $A$. We say that $\mathcal{A}_{G}$ is the Hopf $*$-algebra associated to $G$. Recall also that the matrix elements $U_{j k}^{\alpha}$ are linearly independent and therefore form a linear basis of $\mathcal{A}$.

The quantum group $G$ admits a Haar integral $h$. When restricted to $\mathcal{A}, h$ is a (positive) Haar integral in the sense of Section 2, 
We denote by $\mathcal{A}_{\alpha}$ the linear span of the set $\left\{U_{j k}^{\alpha} \mid j, k=1, \ldots, N_{\alpha}\right\}$. If $\chi$ is a linear functional on $\mathcal{A}$, then $\mathcal{A}_{\alpha}$ is invariant for the operator $E_{\chi}=(\mathrm{id} \otimes \chi) \Delta$. This follows from the calculation $E_{\chi}\left(U_{j k}^{\alpha}\right)=(\mathrm{id} \otimes \chi) \Delta\left(U_{j k}^{\alpha}\right)=(\mathrm{id} \otimes \chi)\left(\sum_{r=1}^{N_{\alpha}} U_{j r}^{\alpha} \otimes U_{r k}^{\alpha}\right)=$ $\sum_{r=1}^{N_{\alpha}} U_{j r}^{\alpha} \chi\left(U_{r k}^{\alpha}\right)$.

A $*$-differential calculus over $G$ is, by definition, a *-differential calculus over the associated Hopf $*$-algebra $\mathcal{A}$. If $(\Omega, d)$ is such a strongly finite-dimensional calculus, set $\Omega(\alpha)=\mathcal{A}_{\alpha} \otimes \Omega^{\text {inv }}$. Then $\Omega(\alpha)$ is a finite-dimensional linear subspace of $\Omega$ and

$$
\Omega=\bigoplus_{\alpha \in \hat{G}} \Omega(\alpha) .
$$

Moreover, if we have a left invariant inner product on $\Omega$, the spaces $\Omega(\alpha)$ are pairwise orthogonal, since the spaces $\mathcal{A}_{\alpha}$ are pairwise orthogonal in $\mathcal{A}$ [11, Theorem 7.4].

From Equations (2) and (3) it follows that $\Omega(\alpha)$ is invariant for both $d$ and $d^{*}$, since $E_{\chi}\left(\mathcal{A}_{\alpha}\right) \subseteq \mathcal{A}_{\alpha}$, for every linear functional $\chi$ on $\mathcal{A}$.

We shall say that an operator $T$ on a linear space $X$ is diagonalisable if $X$ admits a linear basis consisting of eigenvectors of $T$.

Condition 3 of the following result is our Hodge decomposition.

Theorem 4.1. Let $(\Omega, d)$ be a strongly finite-dimensional *-differential calculus over a compact quantum group $G$ and suppose we have a graded, left invariant inner product on $\Omega$. Then

1. The Dirac operator $D$ and the Laplacian $\nabla$ are diagonalisable.

2. We have $\operatorname{ker}(\nabla)=\operatorname{ker}(D)=\operatorname{ker}(d) \cap \operatorname{ker}\left(d^{*}\right)$ and, if $t$ is a positive eigenvalue of $\nabla$, then

$$
\operatorname{ker}(\nabla-t)=\operatorname{ker}(D+\sqrt{t}) \oplus \operatorname{ker}(D-\sqrt{t})
$$

3. The space $\Omega$ admits the orthogonal decomposition

$$
\Omega=\Omega_{\nabla} \oplus d(\Omega) \oplus d^{*}(\Omega) .
$$

Proof. First, let $t$ be a non-zero real number and let $t^{2}$ be an eigenvalue of $\nabla$. Let $\omega$ be a corresponding eigenvector and set $\omega_{ \pm}=\omega \pm t^{-1} D(\omega)$. If $\omega_{+}$and $\omega_{-}$are non-zero, then a simple computation shows that $\omega_{+}$and $\omega_{-}$are eigenvectors of $D$, with $t$ and $-t$ as the corresponding eigenvalues. If $\omega_{+}=0$, then $D(\omega)=-t \omega$. Similarly, if $\omega_{-}=0$, then $D(\omega)=t \omega$. In any case, whether $\omega_{+}$or $\omega_{-}$are zero or non-zero, we can use the fact that $\omega=\left(\omega_{+}+\omega_{-}\right) / 2$ to deduce that $\operatorname{ker}\left(\nabla-t^{2}\right)=$ $\operatorname{ker}(D+t) \oplus \operatorname{ker}(D-t)$. We shall presently show that $\operatorname{ker}(\nabla)=\operatorname{ker}(D)$.

As we observed in the remarks preceding this theorem, each subspace $\Omega(\alpha)$ of $\Omega$ is invariant for both $d$ and $d^{*}$. If $d_{\alpha}$ and $d_{\alpha}^{*}$ denote the restrictions of $d$ and $d^{*}$ to $\Omega(\alpha)$, it is clear that $d_{\alpha}^{*}$ is the adjoint of $d_{\alpha}$. Let $D_{\alpha}=d_{\alpha}+d_{\alpha}^{*}$ be the restriction of $D=d+d^{*}$ to $\Omega(\alpha)$. Since $D_{\alpha}$ is a self-adjoint operator on a finite-dimensional space, its matrix with respect to a suitable basis is diagonal. This implies easily that $\operatorname{ker}\left(D_{\alpha}^{2}\right)=\operatorname{ker}\left(D_{\alpha}\right)$. These observations show that $\Omega_{\nabla}=\operatorname{ker}(\nabla)=\operatorname{ker}(D)$. On the other hand, the spaces $d(\Omega)$ and $d^{*}(\Omega)$ are orthogonal, since $d^{2}=0$. Hence, if $D(\omega)=0$, then $d \omega=-d^{*} \omega$, and therefore, $d \omega=0=d^{*} \omega$. It follows that $\operatorname{ker}(D)=\operatorname{ker}(d) \cap \operatorname{ker}\left(d^{*}\right)$. Hence, Condition 2 holds.

Also, $\left(d_{\alpha} d_{\alpha}^{*}\right)\left(d_{\alpha}^{*} d_{\alpha}\right)=0=\left(d_{\alpha}^{*} d_{\alpha}\right)\left(d_{\alpha} d_{\alpha}^{*}\right)$. Hence, $d_{\alpha} d_{\alpha}^{*}$ and $d_{\alpha}^{*} d_{\alpha}$ are commuting (positive) normal operators on the finite-dimensional space $\Omega(\alpha)$ and are therefore simultaneously diagonalisable. It follows from Equation (4) that $\Omega$ admits a linear 
basis $\left(e_{i}\right)_{i \in I}$ of simultaneous eigenvectors of $d d^{*}$ and $d^{*} d$, implying that $\nabla$ is diagonalisable. Since Condition 2 holds, this also implies that $D_{\alpha}$ is diagonalisable, and we have proven Condition 1.

To prove Condition 3 , we first observe that $\Omega_{\nabla}$ is orthogonal to the spaces $d(\Omega)$ and $d^{*}(\Omega)$. For, if $\omega \in \Omega_{\nabla}$ and $\omega^{\prime}, \omega^{\prime \prime} \in \Omega$, then $\left(d\left(\omega^{\prime}\right)+d^{*}\left(\omega^{\prime \prime}\right) \mid \omega\right)=$ $\left(\omega^{\prime} \mid d^{*} \omega\right)+\left(\omega^{\prime \prime} \mid d \omega\right)=0$, since $d \omega=d^{*} \omega=0$, by Condition 2 .

Now let $\lambda_{i}$ and $\mu_{i}$ be scalars such that $\left(d d^{*}\right)\left(e_{i}\right)=\lambda_{i} e_{i}$ and $\left(d^{*} d\right)\left(e_{i}\right)=\mu_{i} e_{i}$. Since $\left(d d^{*}\right)\left(d^{*} d\right)=0$, we have $\lambda_{i} \mu_{i}=0$, for all $i \in I$. If $\lambda_{i} \neq 0$, then $e_{i} \in d(\Omega)$. Similarly, if $\mu_{i} \neq 0$, then $e_{i} \in d^{*}(\Omega)$. On the other hand, if $\lambda_{i}=\mu_{i}=0$, then $e_{i} \in \Omega_{\nabla}$. This shows that $\Omega=\Omega_{\nabla}+d(\Omega)+d^{*}(\Omega)$, and Condition 3 holds.

Corollary 4.2. The orthogonal complements of $d(\Omega)$ and $d^{*}(\Omega)$ in $\Omega$ are given by

$$
d(\Omega)^{\perp}=\Omega_{\nabla} \oplus d^{*}(\Omega)=\operatorname{ker}\left(d^{*}\right)=\operatorname{ker}\left(d d^{*}\right)
$$

and

$$
d^{*}(\Omega)^{\perp}=\Omega_{\nabla} \oplus d(\Omega)=\operatorname{ker}(d)=\operatorname{ker}\left(d^{*} d\right) .
$$

Proof. We consider only the case of $d(\Omega)^{\perp}$ (the case for $d^{*}(\Omega)^{\perp}$ is handled the same way). It is clear from the theorem that $d(\Omega)^{\perp}=\Omega_{\nabla} \oplus d^{*}(\Omega)$ and it follows directly from the definition of $d(\Omega)^{\perp}$ that $d(\Omega)^{\perp}=\operatorname{ker}\left(d^{*}\right) \subseteq \operatorname{ker}\left(d d^{*}\right)$. Then suppose $\omega \in \operatorname{ker}\left(d d^{*}\right)$. By the theorem, we may write $\omega=\omega_{0}+d \omega_{1}+d^{*} \omega_{2}$, where $\omega_{0} \in \Omega_{\nabla}$ and $\omega_{1}, \omega_{2} \in \Omega$. Then $0=d d^{*}(\omega)=d d^{*}\left(\omega_{0}\right)+d d^{*} d\left(\omega_{1}\right)=-d^{*} d\left(\omega_{0}\right)+d d^{*} d\left(\omega_{1}\right)$, since $\nabla\left(\omega_{0}\right)=0$. Hence, $d^{*} d\left(\omega_{0}\right)=d d^{*} d\left(\omega_{1}\right)$ belongs to $d^{*}(\Omega)$ and to $d(\Omega)$, and is therefore equal to zero. It follows that $\nabla\left(d \omega_{1}\right)=d d^{*} d\left(\omega_{1}\right)=0$. Consequently, $d\left(\omega_{1}\right)=0$. Hence, $\omega=\omega_{0}+d^{*}\left(\omega_{2}\right) \in d(\Omega)^{\perp}$. Therefore, $\operatorname{ker}\left(d d^{*}\right)=d(\Omega)^{\perp}$.

\section{Diagonalising $\nabla$ ON The 0 - AND $N$-FORMS}

Throughout this section we denote by $(\Omega, d)$ a strongly finite-dimensional *differential calculus of dimension $N$ over the Hopf $*$-algebra $\mathcal{A}$ associated to a compact quantum group $G$. We suppose that $\operatorname{dim}\left(\Omega_{N}^{\text {inv }}\right)=1$ and that $d\left(\Omega_{N-1}^{\text {inv }}\right)=0$. We fix a volume element $\theta$ in $\Omega_{N}^{\text {inv }}$ and denote by $h$ the Haar measure of $\mathcal{A}$. We also fix a graded, left invariant inner product (. . . ) on $\Omega$.

The representations $U^{\alpha}$ are as in the first paragraph of Section 4.

Set $\chi=\sum_{r=1}^{M} \chi_{r}^{*} \chi_{r}$, where $\omega_{1}, \ldots, \omega_{M}$ and $\chi_{1}, \ldots, \chi_{M}$ are chosen as in Equation (11). Multiplication of linear functionals $f$ and $g$ on $\mathcal{A}$ is given, as usual, by setting $(f g)(a)=(f \otimes g) \Delta(a)$.

Theorem 5.1. If $a \in \mathcal{A}$, then $\nabla a=\chi * a$.

Proof. Applying Equation (3) to Equation (10), we get

$$
d^{*} d a=\sum_{s, r=1}^{M} E_{\chi_{s}^{*} \chi_{r}}(a) M_{\omega_{s}}^{*}\left(\omega_{r}\right)+\sum_{r=1}^{M} E_{\chi_{r}}(a) d^{*}\left(\omega_{r}\right) .
$$

Since $d 1=0, d^{*}\left(\Omega_{1}^{\text {inv }}\right)=0$; hence, the second sum above vanishes. It is trivially verified that $M_{\omega_{s}}^{*}\left(\omega_{r}\right)=\delta_{r s}$. Hence, $d^{*} d a=\sum_{r=1}^{M} E_{\chi_{r}^{*} \chi_{r}}(a)=E_{\chi}(a)$. Since $d^{*} a=0$, it follows that $\nabla a=\chi * a$.

The formula for $\nabla$ in the preceding theorem shows that $\chi$ does not depend on the choice of orthonormal basis $\omega_{1}, \ldots, \omega_{M}$ of $\Omega_{1}^{\text {inv }}$.

Lemma 5.2. The operator $T^{\alpha}=(\mathrm{id} \otimes \chi) \Delta\left(U^{\alpha}\right) \in B\left(H_{\alpha}\right)$ is necessarily positive. 
Proof. If $\tau$ and $\sigma$ are linear functionals on $\mathcal{A}$, it follows easily from the basic representation theory that $(\mathrm{id} \otimes \tau)\left(U^{\alpha}\right)(\mathrm{id} \otimes \sigma)\left(U^{\alpha}\right)=(\mathrm{id} \otimes \tau \sigma)\left(U^{\alpha}\right)$ and $\left((\operatorname{id} \otimes \tau)\left(U^{\alpha}\right)\right)^{*}$ $=\left(\mathrm{id} \otimes \tau^{*}\right)\left(U^{\alpha}\right)$. Therefore, we have $T^{\alpha}=(\mathrm{id} \otimes \chi)\left(U^{\alpha}\right)=\sum_{r=1}^{M}\left(\mathrm{id} \otimes \chi_{r}^{*} \chi_{r}\right)\left(U^{\alpha}\right)=$ $\sum_{r=1}^{M}\left(\left(\mathrm{id} \otimes \chi_{r}\right)\left(U^{\alpha}\right)\right)^{*}\left(\mathrm{id} \otimes \chi_{r}\right)\left(U^{\alpha}\right)$. Thus, $T^{\alpha}$ is a sum of positive operators and is therefore itself positive.

Theorem 5.3. Let $\left(U^{\alpha}\right)_{\alpha}$ be a complete family of mutually inequivalent irreducible unitary representations of the compact quantum group $G$. Let $T^{\alpha}=(\mathrm{id} \otimes \chi) \Delta\left(U^{\alpha}\right)$. Choose an orthonormal basis $e_{1}^{\alpha}, \ldots, e_{N_{\alpha}}^{\alpha}$ for the space $H_{\alpha}$ on which $U^{\alpha}$ acts making $T^{\alpha}$ diagonal, so that $T^{\alpha} e_{i}^{\alpha}=\lambda_{i}^{\alpha} e_{i}^{\alpha}$, for some elements $\lambda_{i}^{\alpha} \in \mathbf{C}$. Let $U_{i j}^{\alpha}$ be the matrix elements of $U^{\alpha}$ relative to this basis. Then $\nabla U_{i j}^{\alpha}=\lambda_{j}^{\alpha} U_{i j}^{\alpha}$.

Proof. By Theorem 5.1, $\nabla U_{i j}^{\alpha}=\chi * U_{i j}^{\alpha}$. Since $\Delta\left(U_{i j}^{\alpha}\right)=\sum_{k=1}^{N_{\alpha}} U_{i k}^{\alpha} \otimes U_{k j}^{\alpha}$, we have $\chi * U_{i j}^{\alpha}=\sum_{k=1}^{N_{\alpha}} U_{i k}^{\alpha} \chi\left(U_{k j}^{\alpha}\right)=\sum_{k=1}^{N_{\alpha}} U_{i k}^{\alpha} T_{k j}^{\alpha}=\sum_{k=1}^{N_{\alpha}} U_{i k}^{\alpha} \lambda_{j}^{\alpha} \delta_{k j}=\lambda_{j}^{\alpha} U_{i j}^{\alpha}$.

Thus, the theorem diagonalises $\nabla_{0}$, the restriction of $\nabla$ to $\mathcal{A}$, since the matrix elements $U_{i j}^{\alpha}$ form a linear basis for $\mathcal{A}$. It is clear from the theorem that the eigenvalues of $\nabla_{0}$ are precisely the eigenvalues of the operators $T^{\alpha}$ and that, once we know the multiplicities of the eigenvalues of these operators, we can calculate very simply the multiplicities of the eigenvalues of $\nabla_{0}$.

Corollary 5.4. The eigenvalue corresponding to the eigenvector $U_{i j}^{\alpha}$ of $\nabla$ is given by $\chi\left(U_{j j}^{\alpha}\right)=\left\|d U_{i j}^{\alpha}\right\|^{2} /\left\|U_{i j}^{\alpha}\right\|^{2}$.

Proof. From the proof of the theorem we have $\nabla U_{i j}^{\alpha}=\lambda_{j}^{\alpha} U_{i j}^{\alpha}$, where $\lambda_{j}^{\alpha}=T_{j j}^{\alpha}=$ $\chi\left(U_{j j}^{\alpha}\right)$. Hence, $\chi\left(U_{j j}^{\alpha}\right)\left(U_{i j}^{\alpha} \mid U_{i j}^{\alpha}\right)=\left(\nabla U_{i j}^{\alpha} \mid U_{i j}^{\alpha}\right)=\left(d^{*} d U_{i j}^{\alpha} \mid U_{i j}^{\alpha}\right)=\left(d U_{i j}^{\alpha} \mid d U_{i j}^{\alpha}\right)$.

Woronowicz defines the compact quantum group $G$ to be connected if Condition 1 of the following theorem is satisfied. The theorem provides two other equivalent conditions.

Theorem 5.5. The following are equivalent conditions:

1. For any element $a \in \mathcal{A}, d a=0$ if, and only if, $a=\lambda 1$, for some $\lambda \in \mathbf{C}$.

2. For any element $a \in \mathcal{A}, \nabla a=0$ if, and only if, $a=\lambda 1$, for some $\lambda \in \mathbf{C}$.

3. If $U^{\alpha} \neq U^{\alpha_{0}}$, the trivial unitary representation of $G$, then the operator $T^{\alpha}=(\mathrm{id} \otimes \chi) \Delta\left(U^{\alpha}\right)$ is invertible.

Proof. Condition 2 clearly implies 1 . Conversely, if Condition 1 holds, then so does 2 , since $\operatorname{ker}(\nabla)=\operatorname{ker}(d) \cap \operatorname{ker}\left(d^{*}\right)$ and $d^{*} a=0$, for all $a \in \mathcal{A}$.

Suppose now that Condition 2 holds and suppose that $U^{\alpha} \neq U^{\alpha_{0}}$ and that $T^{\alpha}$ is not invertible. Then $\lambda_{j}^{\alpha}=0$, for some $j$ and therefore $\nabla U_{j j}^{\alpha}=\lambda_{j}^{\alpha} U_{j j}^{\alpha}=0$. Hence, by Condition 2, there exists a scalar $c$ for which $U_{j j}^{\alpha}=c 1$. However, since $U^{\alpha} \neq U^{\alpha_{0}}$, the element $U_{j j}^{\alpha}$ is orthogonal to 1 in $\mathcal{A}$. Therefore, $c=h\left(1^{*} U_{j j}^{\alpha}\right)=0$. Thus, $U_{j j}^{\alpha}=0$, an impossibility. To avoid contradiction it must therefore be the case that $T^{\alpha}$ is invertible. Hence, Condition 2 implies 3.

Finally, suppose that Condition 3 holds. Let $a \in \mathcal{A}$ be such that $\nabla a=0$. Because the $U_{i j}^{\alpha}$ form a linear basis of $\mathcal{A}$, we may write $a=\sum_{\alpha} \sum_{i, j=1}^{N_{\alpha}} c_{i j}^{\alpha} U_{i j}^{\alpha}$, for some scalars $c_{i j}^{\alpha}$. Since $0=\nabla a=\sum_{\alpha} \sum_{i, j=1}^{N_{\alpha}} c_{i j}^{\alpha} \lambda_{j}^{\alpha} U_{i j}^{\alpha}$, we have $c_{i j}^{\alpha} \lambda_{j}^{\alpha}=0$, for all $\alpha, i$ and $j$. Hence, if $U^{\alpha} \neq U^{\alpha_{0}}$, then $\lambda_{j}^{\alpha} \neq 0$, and therefore $c_{i j}^{\alpha}=0$. It follows that $a=c 1$, for $c=c_{11}^{\alpha_{0}}$. Hence, Condition 3 implies 2 . 
We turn now to finding the eigenvalues and eigenvectors of the Laplacian on the highest-dimensional forms, the $N$-forms. The situation here turns out to be analogous to that for the lowest-dimensional forms, the 0 -forms.

We first need to introduce a new linear functional $\gamma$ on $\mathcal{A}$, an analogue for the case of the $N$-forms of the functional $\chi$ used for the case of the 0 -forms. It is clear, since $\Omega_{N}^{\mathrm{inv}}=\mathbf{C} \theta$, that $M_{\omega_{r}} M_{\omega_{s}}^{*}(\theta)=c_{r s} \theta$, for some scalars $c_{r s}$. Since $c_{r s}=\left(M_{\omega_{r}} M_{\omega_{s}}^{*} \theta \mid \theta\right)=\left(M_{\omega_{s}}^{*} \theta \mid M_{\omega_{r}}^{*} \theta\right)$, the matrix $\left(c_{r s}\right)$ is positive. We set $\gamma=\sum_{r, s=1}^{M} c_{r s} \chi_{r} \chi_{s}^{*}$

Theorem 5.6. If $a \in \mathcal{A}$, then $\nabla(a \theta)=(\gamma * a) \theta$.

Proof. Note first that $d(a \theta)=0$, since $d\left(\Omega_{N}\right)=0$. Hence, $\nabla(a \theta)=d d^{*}(a \theta)$. Using Equation (3), we get $d^{*}(a \theta)=a d^{*}(\theta)+\sum_{s=1}^{M} E_{\chi_{s}^{*}}(a) M_{\omega_{s}}^{*}(\theta)$. The first term here is equal to zero, since $d^{*}(\theta)=0$; this is an immediate consequence of the fact that $d\left(\Omega_{N-1}^{\text {inv }}\right)=0$. It follows, using Equation (2), that $d d^{*}(a \theta)=$ $\sum_{s=1}^{M} E_{\chi_{s}^{*}}(a) d M_{\omega_{s}}^{*}(\theta)+\sum_{r, s=1}^{M} E_{\chi_{r} \chi_{s}^{*}}(a) M_{\omega_{r}} M_{\omega_{s}}^{*}(\theta)$. Again using the fact that $d\left(\Omega_{N-1}^{\text {inv }}\right)=0$, we see that the first sum vanishes. Since $M_{\omega_{r}} M_{\omega_{s}}^{*}(\theta)=c_{r s} \theta$, we have $\nabla(a \theta)=d d^{*}(a \theta)=\sum_{r, s=1}^{M} c_{r s} E_{\chi_{r} \chi_{s}^{*}}(a) \theta=(\gamma * a) \theta$.

The formula for $\nabla$ restricted to the $N$-forms in the preceding proposition shows that $\gamma$ does not depend on the choice of orthonormal basis $\omega_{1}, \ldots, \omega_{M}$ of $\Omega_{1}^{\text {inv }}$.

Lemma 5.7. The operator $S^{\alpha}=(\mathrm{id} \otimes \gamma)\left(U^{\alpha}\right) \in B\left(H_{\alpha}\right)$ is necessarily positive.

Proof. We have $S^{\alpha}=\sum_{r, s=1}^{M} c_{r s}\left(\mathrm{id} \otimes \chi_{r}\right)\left(U^{\alpha}\right)\left(\left(\mathrm{id} \otimes \chi_{s}\right)\left(U^{\alpha}\right)\right)^{*}$. Also, since the matrix $\left(c_{r s}\right)$ is positive, it can be written as a sum of matrices $c^{1}, \ldots, c^{K}$, with each such matrix of the form $c_{r s}^{k}=c_{r}^{k} \bar{c}_{s}^{k}$, for some scalars $c_{r}^{k}$. Hence,

$$
\begin{aligned}
S^{\alpha} & =\sum_{k=1}^{K} \sum_{r, s=1}^{M} c_{r}^{k} \bar{c}_{s}^{k}\left(\mathrm{id} \otimes \chi_{r}\right)\left(U^{\alpha}\right)\left(\left(\mathrm{id} \otimes \chi_{s}\right)\left(U^{\alpha}\right)\right)^{*} \\
& =\sum_{k=1}^{K}\left(\sum_{r=1} c_{r}^{k}\left(\mathrm{id} \otimes \chi_{r}\right)\left(U^{\alpha}\right)\right)\left(\sum_{r=1} c_{r}^{k}\left(\mathrm{id} \otimes \chi_{r}\right)\left(U^{\alpha}\right)\right)^{*} .
\end{aligned}
$$

Thus, $S^{\alpha}$ is a sum of positive operators and is therefore itself positive.

Theorem 5.8. Let $\left(U^{\alpha}\right)_{\alpha}$ be a complete family of mutually inequivalent irreducible unitary representations of the compact quantum group $G$. Set $S^{\alpha}=(\mathrm{id} \otimes \gamma)\left(U^{\alpha}\right)$ and choose an orthonormal basis $e_{1}^{\alpha}, \ldots, e_{N_{\alpha}}^{\alpha}$ for the space $H_{\alpha}$ on which $U^{\alpha}$ acts making $S^{\alpha}$ diagonal, so that $S^{\alpha} e_{i}^{\alpha}=\lambda_{i}^{\alpha} e_{i}^{\alpha}$, for some elements $\lambda_{i}^{\alpha} \in \mathbf{C}$. Let $U_{i j}^{\alpha}$ be the matrix elements of $U^{\alpha}$ relative to this basis. Then $\nabla\left(U_{i j}^{\alpha} \theta\right)=\lambda_{j}^{\alpha} U_{i j}^{\alpha} \theta$.

Proof. By Theorem [5.6. $\nabla\left(U_{i j}^{\alpha} \theta\right)=\left(\gamma * U_{i j}^{\alpha}\right) \theta$. Since $\gamma * U_{i j}^{\alpha}=\sum_{k=1}^{N_{\alpha}} U_{i k}^{\alpha} \gamma\left(U_{k j}^{\alpha}\right)=$ $\sum_{k=1}^{N_{\alpha}} U_{i k}^{\alpha} S_{k j}^{\alpha}=\sum_{k=1}^{N_{\alpha}} U_{i k}^{\alpha} \lambda_{j}^{\alpha} \delta_{k j}=\lambda_{j}^{\alpha} U_{i j}^{\alpha}$, we have $\nabla\left(U_{i j}^{\alpha} \theta\right)=\lambda_{j}^{\alpha} U_{i j}^{\alpha} \theta$, as required.

Remarks analogous to those made after Theorem 5.3 apply to Theorem 5.8

We now show that a formula holds for the Laplacian applied to general $k$-forms that is analogous to those we obtained in Theorems 5.1] and 5.6 for 0-forms and $N$-forms, respectively.

Before proceeding, note that $\mathcal{A}_{\alpha}$ is finite dimensional, of dimension $N_{\alpha}^{2}$. Let $k$ be an integer such that $0 \leq k \leq N$. The linear span $\Omega_{\alpha, k}$ of the forms $a \omega$, where 
$a \in \mathcal{A}_{\alpha}$ and $\omega \in \Omega_{k}^{\text {inv }}$, is linearly isomorphic to the tensor product $\mathcal{A}_{\alpha} \otimes \Omega_{k}^{\text {inv }}$, where the isomorphism maps $a \omega$ onto $a \otimes \omega$. We shall use this isomorphism to identify these two spaces. Using the fact that the matrix entries of $U^{\alpha}$ are orthogonal to the matrix entries of $U^{\beta}$, if $\alpha \neq \beta$, it is clear that $\Omega_{k}=\bigoplus_{\alpha} \Omega_{\alpha, k}$, as an orthogonal sum.

Equations (2) and (3) imply that the space $\Omega_{k}=\mathcal{A} \otimes \Omega_{k}^{\text {inv }}$ is invariant for $\nabla$; we may therefore write $\nabla=\nabla_{0} \oplus \cdots \oplus \nabla_{N}$, where $\nabla_{k}$ is the restriction of $\nabla$ to $\Omega_{k}$. Similarly, the spaces $\Omega_{\alpha, k}$ are also invariant for $\nabla$. Hence, $\nabla_{k}=\bigoplus_{\alpha} \nabla_{\alpha, k}$, where $\nabla_{\alpha, k}$ is the restriction of $\nabla$ to $\Omega_{\alpha, k}$. We therefore reduce the eigenvalue problem for $\nabla$ to one for the finite-dimensional operator $\nabla_{\alpha, k}$.

Recall, for the proof of the following theorem, the elementary fact that if $\left(e_{i}\right)_{i \in I}$ is a finite linear basis for a linear space $X$, the corresponding system of matrix units $\left(e_{i j}\right)_{i, j \in I}$ in $B(X)$ is defined by setting $e_{i j}\left(e_{k}\right)=\delta_{j k} e_{i}$ and that if $T \in B(X)$ has matrix $\left(\lambda_{i j}\right)$ relative to the basis $\left(e_{i}\right)$, then $T=\sum_{i, j \in I} \lambda_{i j} e_{i j}$.

Theorem 5.9. Suppose $k$ is an integer such that $0 \leq k \leq N$ and let $\eta_{1}, \ldots, \eta_{L}$ be a linear basis for $\Omega_{k}^{\mathrm{inv}}$.

1. For $p, q=1, \ldots, L$, there exist unique linear functionals $g_{p q}$ on $\mathcal{A}$ such that

$$
\nabla\left(a \eta_{p}\right)=\sum_{q=1}^{L}\left(g_{q p} * a\right) \eta_{q}
$$

for all $a \in \mathcal{A}$.

2. If $\alpha \in \hat{G}$, the restriction $\nabla_{\alpha, k}$ of $\nabla$ to $\Omega_{\alpha, k}$ is similar to a direct sum of $N_{\alpha}$ copies of the block matrix

$$
\left[\begin{array}{cccc}
g_{11}\left(U^{\alpha}\right) & g_{12}\left(U^{\alpha}\right) & \ldots & g_{1 L}\left(U^{\alpha}\right) \\
g_{21}\left(U^{\alpha}\right) & g_{22}\left(U^{\alpha}\right) & \ldots & g_{2 L}\left(U^{\alpha}\right) \\
\vdots & \vdots & \ddots & \vdots \\
g_{L 1}\left(U^{\alpha}\right) & g_{L 2}\left(U^{\alpha}\right) & \ldots & g_{L L}\left(U^{\alpha}\right)
\end{array}\right]
$$

where $g_{p q}\left(U^{\alpha}\right)$ is the $N_{\alpha} \times N_{\alpha}$-matrix with ijth entry $g_{p q}\left(U_{i j}^{\alpha}\right)$.

Proof. Let $M_{r}^{k}$ denote the operator from $\Omega_{k}^{\text {inv }}$ to $\Omega_{k+1}^{\text {inv }}$ of left multiplication by $\omega_{r}$. Let $\chi_{0}$ be the co-unit of $\mathcal{A}$ and let $M_{0}^{k}$ be the restriction of $d$ to $\Omega_{k}^{\text {inv }}$. Finally, set $E_{r}=E_{\chi_{r}}$. By Theorem 2.4, $\nabla_{k}=\sum_{r, s=0}^{M} E_{r} E_{s}^{*} \otimes M_{r}^{k-1}\left(M_{s}^{k-1}\right)^{*}+$ $\sum_{r, s=0}^{M} E_{r}^{*} E_{s} \otimes\left(M_{r}^{k}\right)^{*} M_{s}^{k}$. Since the operators $M_{r}^{k-1}\left(M_{s}^{k-1}\right)^{*}$ and $\left(M_{r}^{k}\right)^{*} M_{s}^{k}$ are linear combinations of the matrix units $\eta_{p q}$ corresponding to the basis $\eta_{1}, \ldots, \eta_{L}$ of $\Omega_{k}^{\text {inv }}$, it follows that $\nabla_{k}=\sum_{p, q=1}^{L} E_{g_{p q}} \otimes \eta_{p q}$, for linear functionals $g_{p q}$ that are linear combinations of the functionals $\chi_{r} \chi_{s}^{*}$ and $\chi_{r}^{*} \chi_{s}$. This proves Condition 1 (uniqueness of the functionals $g_{p q}$ is clear).

In the remainder of this proof, in summations the indices $p, q$ will range over the numbers $1, \ldots, L$ and the indices $i, j$ over the range $1, \ldots, N_{\alpha}$. For $m \in\left\{1, \ldots, N_{\alpha}\right\}$, we have $\nabla\left(U_{m i}^{\alpha} \eta_{p}\right)=\sum_{q}\left(g_{q p} * U_{m i}^{\alpha}\right) \eta_{q}=\sum_{q, j} U_{m j}^{\alpha} g_{q p}\left(U_{j i}^{\alpha}\right) \eta_{q}$. Hence, if $Y=\Omega_{\alpha, k}$ and $X_{m}$ is the linear span of the elements $U_{m 1}^{\alpha}, \ldots, U_{m N_{\alpha}}^{\alpha}$, then $Y_{m}=X_{m} \otimes \Omega_{k}^{\text {inv }}$ is an invariant subspace for $\nabla$ and $Y=Y_{1} \oplus \cdots \oplus Y_{N_{\alpha}}$. We write $T_{m}$ for the restriction of $\nabla$ to $Y_{m}$. Then $\nabla_{\alpha, k}=T_{1} \oplus \cdots \oplus T_{N_{\alpha}}$. We shall show that each summand is similar to the block matrix displayed in Condition 2, and this will complete the proof of the theorem. 
Let $e_{i}=U_{m i}^{\alpha}$, for $i=1, \ldots, N_{\alpha}$. Then the $e_{i}$ form a basis for $X_{m}$ and therefore the elements $e_{i} \otimes \eta_{p}$ form a basis for $Y_{m}$. Let $e_{i j}, \eta_{p q}$ and $f_{(i, p),(j, q)}$ form a system of matrix units corresponding to the bases $e_{i}, \eta_{p}$ and $e_{i} \otimes \eta_{p}$, respectively. It is elementary to verify that $f_{(i, p),(j, q)}=e_{i j} \otimes \eta_{p q}$. Since $\nabla\left(U_{m j}^{\alpha} \eta_{q}\right)=\sum_{p, i} U_{m i}^{\alpha} g_{p q}\left(U_{i j}^{\alpha}\right) \eta_{p}$, we have

$$
T_{m}=\sum_{i j p q} g_{p q}\left(U_{i j}^{\alpha}\right) f_{(i, p),(j, q)}=\sum_{p q}\left(\sum_{i j} g_{p q}\left(U_{i j}^{\alpha}\right) e_{i j}\right) \otimes \eta_{p q}
$$

Hence, $T_{m}$ has matrix with respect to the basis $e_{i} \otimes \eta_{p}$ of $Y_{m}$ the block matrix displayed in Condition 2 and therefore $T_{m}$ is similar to that matrix, as required.

Thus, the eigenvalue problem for the Laplacian $\nabla$ has been reduced to the finitedimensional problem of finding the eigenvalues of the block matrix in Condition 2 of Theorem 5.9. However, this matrix is of order $N_{\alpha} L$; it may be difficult to work with in practice, since, in general, $N_{\alpha}$ can be arbitrarily large. In Section 8 we present an alternative approach in a special case.

\section{The InNer PRODUCT ON $\Omega^{\text {inv }}$}

In this section we make the same assumptions and notational conventions as in the first paragraph of Section 3 without fixing an inner product on $\Omega$. In addition, we suppose that $\Omega$ is non-degenerate and that $\int$ is the integral associated to $\theta$.

In general, the Laplacian $\nabla$ and the Hodge operator $L$ need not commute, as one can see by looking at examples. However, when they do commute, the problem of computing the eigenvalues of $\nabla$ is effectively halved. For, if $\omega \in \Omega_{k}$ and $\nabla \omega=\lambda \omega$, for some scalar $\lambda$, then $\nabla L(\omega)=\lambda L(\omega)$ also. Thus, if one computes the eigenvalues of $\nabla$ on $\Omega_{k}$, one knows them for $\nabla$ on $\Omega_{N-k}$ also, where $N$ is the dimension of $\Omega$.

Up to this point, we have not specified how the inner product on $\Omega^{\text {inv }}$ should be chosen, except to say that it should be graded. It turns out that one can indeed choose the inner product on $\Omega^{\text {inv }}$ in such a way as to ensure that $\nabla L=L \nabla$. We turn now to showing this.

Choose an inner product $(\cdot \mid \cdot)^{\prime}$ on $\Omega^{\text {inv }}$ and let $L^{\prime}$ be the corresponding Hodge operator on $\Omega$. We obtain a new graded inner product $(\cdot \mid \cdot)$ on $\Omega^{\text {inv }}$ by defining $\left(\omega_{1} \mid \omega_{2}\right)$, for $\omega_{1}, \omega_{2} \in \Omega_{k}^{\text {inv }}$, as follows:

1. If $k<N / 2$, set $\left(\omega_{1} \mid \omega_{2}\right)=\left(\omega_{1} \mid \omega_{2}\right)^{\prime}$.

2. If $k>N / 2$, set $\left(\omega_{1} \mid \omega_{2}\right)=\left(\left(L^{\prime}\right)^{-1}\left(\omega_{1}\right) \mid\left(L^{\prime}\right)^{-1}\left(\omega_{2}\right)\right)^{\prime}$.

For the moment, we set aside the case of $k=N / 2$, which occurs, of course, only if $N$ is even. We suppose only that the new inner product has been specified in some way on $\Omega_{N / 2}^{\text {inv }}$ in this case.

Note that the new inner product on $\Omega_{N}^{\text {inv }}$ is the same as the old (since $L^{\prime}(1)=\theta$ ). Hence, $\theta$ is still a volume element for $\Omega$ with its new inner product.

Let $L$ be the Hodge operator on $\Omega$ associated to this new inner product.

Observe that, if $k<N / 2$, then $L=L^{\prime}$ on $\Omega_{k}$. This follows immediately from faithfulness of $\int$.

On the other hand, if $k>N / 2$, then $L=(-1)^{k(N-k)}\left(L^{\prime}\right)^{-1}$ on $\Omega_{k}$. To see this, we need only show that $L(\omega)=(-1)^{k(N-k)}\left(L^{\prime}\right)^{-1}(\omega)$, for $\omega \in \Omega_{k}^{\text {inv }}$. Hence, we need only show $\int \eta^{*}\left(L(\omega)-(-1)^{k(N-k)}\left(L^{\prime}\right)^{-1}(\omega)\right)=0$, for all $\eta \in \Omega_{k}^{\text {inv }}$, by faithfulness 
of $\int$. To see this equality, observe first that if $\omega \in \Omega_{N-k}^{\text {inv }}$ and $\eta \in \Omega_{k}^{\text {inv }}$, then

$$
\begin{aligned}
& \left(\left(L^{\prime}\right)^{-1}(\omega) \mid \eta\right)^{\prime}=\int \eta^{*} \omega=\overline{\int\left(\eta^{*} \omega\right)^{*}}=(-1)^{k(N-k) \overline{\int \omega^{*} \eta}} \\
& \quad=(-1)^{k(N-k)} \frac{\overline{\left(\left(L^{\prime}\right)^{-1}(\eta) \mid \omega\right)^{\prime}}}{=(-1)^{k(N-k)}\left(\omega \mid\left(L^{\prime}\right)^{-1}(\eta)\right)^{\prime} .}
\end{aligned}
$$

Hence, if $\eta, \omega \in \Omega_{k}^{\text {inv }}$, then $\int \eta^{*} L(\omega)=(\omega \mid \eta)=\left(\left(L^{\prime}\right)^{-1}(\omega) \mid\left(L^{\prime}\right)^{-1}(\eta)\right)^{\prime}=$ $(-1)^{k(N-k)}\left(\left(L^{\prime}\right)^{-2}(\omega) \mid \eta\right)^{\prime}=(-1)^{k(N-k)} \int \eta^{*}\left(L^{\prime}\right)^{-1}(\omega)$. Therefore, as required, we have $\int \eta^{*}\left(L(\omega)-(-1)^{k(N-k)}\left(L^{\prime}\right)^{-1}(\omega)\right)=0$.

It follows from these calculations that $L^{2}(\omega)=(-1)^{k(N-k)} \omega$, for all $\omega \in \Omega_{k}$, if $k \neq N / 2$. We now show how to choose the inner product on $\Omega_{k}^{\text {inv }}$, where $k=N / 2$, in such a way that, in this case also, $L^{2}(\omega)=(-1)^{k(N-k)} \omega$, for all $\omega \in \Omega_{k}$.

The sesquilinear form, $(\eta, \omega) \mapsto \int \omega^{*} \eta$, is non-degenerate on $\Omega_{k}^{\text {inv }}$, by faithfulness of $\int$. Also, this form is Hermitian if $(-1)^{k(N-k)}=1$ and anti-Hermitian if $(-1)^{k(N-k)}=-1$. Set $\varepsilon_{k}=1$ in the first case and $\varepsilon_{k}=i$ in the second. Then there exists a basis $e_{1}, \ldots, e_{m}$ for $\Omega_{k}^{\text {inv }}$ such that $\int e_{q}^{*} e_{p}= \pm \varepsilon_{k} \delta_{p q}$, for all $p, q=1, \ldots, m$. Now choose an inner product on $\Omega_{k}^{\text {inv }}$ making $e_{1}, \ldots, e_{m}$ orthonormal. For this inner product, the operator $L$ on $\Omega_{k}^{\text {inv }}$ defined by mapping $e_{p}$ onto $(-1)^{k(N-k)}\left(\int e_{p}^{*} e_{p}\right) e_{p}$, for $p=1, \ldots, m$, satisfies the condition $\int \eta^{*} L(\omega)=(\omega \mid \eta)$. Hence, $L$ is the Hodge operator on $\Omega_{k}^{\text {inv }}$ and extends in an obvious way to the Hodge operator on $\Omega_{k}$. Clearly, $L^{2}(\omega)=(-1)^{k(N-k)} \omega$, for all $\omega \in \Omega_{k}$.

It is clear from our construction of $L$ that it is a unitary operator on $\Omega$.

We sum up what we have shown here in the following theorem and draw out some useful consequences.

Theorem 6.1. Let $(\Omega, d)$ be a non-degenerate strongly finite-dimensional $*$-differential calculus of dimension $N$ over a Hopf algebra $\mathcal{A}$ admitting a Haar integral. Suppose that $\operatorname{dim}\left(\Omega_{N}^{\mathrm{inv}}\right)=1$ and $d\left(\Omega_{N-1}^{\mathrm{inv}}\right)=0$. Then $\Omega^{\mathrm{inv}}$ admits an inner product such that the corresponding Hodge operator $L$ is a unitary and satisfies the following conditions:

1. $L^{2}(\omega)=(-1)^{k(N-k)} \omega$, for all $\omega \in \Omega_{k}$.

2. $L d(\omega)=(-1)^{k+1} d^{*} L(\omega)$, for all $\omega \in \Omega_{k}$.

3. $d^{*} d L=L d d^{*}, d d^{*} L=L d^{*} d$ and $L \nabla=\nabla L$.

Proof. Condition 1 was proved above. To show 2, first observe that we may suppose that $k<N$. Now observe that, by Theorem 3.3, $d \omega=(-1)^{N-k} L d^{*} L^{-1}(\omega)$. Hence,

$$
L^{-1} d \omega=(-1)^{N-k} d^{*} L^{-1}(\omega) .
$$

Now, by Condition 1, we have

$$
\begin{gathered}
(-1)^{(k+1)(N-k-1)} L d \omega=L^{-1} d \omega=(-1)^{N-k} d^{*} L^{-1}(\omega) \text { (by Equation (5) ) } \\
=(-1)^{N-k}(-1)^{k(N-k)} d^{*} L(\omega) \text { (by Condition } 1 \text { again). }
\end{gathered}
$$

Hence, $L d \omega=(-1)^{(k+1)(N-k-1)}(-1)^{N-k}(-1)^{k(N-k)} d^{*} L(\omega)=(-1)^{k+1} d^{*} L(\omega)$. Thus, Condition 2 holds.

Again with $\omega \in \Omega_{k}$, we get $d^{*} d L(\omega)=(-1)^{k} d^{*} L d^{*} \omega=(-1)^{k}(-1)^{k} L d d^{*} \omega$, by Theorem 3.3 and Condition 2. Hence, $d^{*} d L=L d d^{*}$. It follows from Condition 1 that $d d^{*} L=L d^{*} d$. Hence, $\nabla L=d d^{*} L+d^{*} d L=L d^{*} d+L d d^{*}=L \nabla$, so Condition 3 holds. 


\section{OnE-PARAMETER REPRESENTATIONS INDUCED BY TWIST AUTOMORPHISMS}

Throughout this section the same standing assumptions and notational conventions will be used as specified in the first paragraph of Section 5], with the added assumption that $\Omega$ is non-degenerate. We will also make use of the notations introduced at the beginning of Section 4 .

The sets of non-negative and positive integers will be denoted by $\mathbf{N}$ and $\mathbf{N}_{0}$, respectively.

In this section we shall need a number of results from the general theory of exponential one-parameter groups of automorphisms. Since these are not completely standard, we have gathered them together in an appendix to which we shall make frequent reference. Therefore we shall use the terminology and notations of the appendix in this section.

We begin by introducing the two most important one-parameter groups connected to the Hopf algebra $\mathcal{A}$. By [14, Theorem 5.6] there exist unique exponential one-parameter groups $\rho$ and $\tau$ on $\mathcal{A}$ such that $h(a b)=h\left(\rho_{i}(b) a\right)$, for all $a, b \in \mathcal{A}$ and $\tau_{i}=\kappa^{-2}$. We call $\rho$ the modular group of $\mathcal{A}$ and of $h$, and $\tau$ the scaling group of $\mathcal{A}$.

There is a family of linear functionals $\left(f_{z}\right)_{z \in \mathbf{C}}$ on $\mathcal{A}$ such that

$$
\rho_{z}(a)=\left(f_{i z} \otimes \operatorname{id}_{\mathcal{A}} \otimes f_{i z}\right) \Delta^{(2)}(a) \quad \text { and } \quad \tau_{z}(a)=\left(f_{i z} \otimes \operatorname{id}_{\mathcal{A}} \otimes f_{-i z}\right) \Delta^{(2)}(a),
$$

for all $z \in \mathbf{C}$ and $a \in \mathcal{A}$. Here we use the usual convention $\Delta^{(2)}=\left(\Delta \otimes \mathrm{id}_{\mathcal{A}}\right) \Delta=$ $\left(\operatorname{id}_{\mathcal{A}} \otimes \Delta\right) \Delta$. (See [14, Theorem 5.6] for these functionals.) It follows that $\rho_{z}\left(\mathcal{A}_{\alpha}\right)=$ $\mathcal{A}_{\alpha}$ and $\tau_{z}\left(\mathcal{A}_{\alpha}\right)=\mathcal{A}_{\alpha}$, for all $\alpha \in \hat{G}$.

We note the following facts: $h \rho_{z}=h \tau_{z}=h$, for all $z \in$ C. Also, $\left(\tau_{z} \otimes \rho_{z}\right) \Delta=$ $\left(\rho_{z} \otimes \tau_{-z}\right) \Delta=\Delta \rho_{z}$ and $\left(\tau_{z} \otimes \tau_{z}\right) \Delta=\left(\rho_{z} \otimes \rho_{-z}\right) \Delta=\Delta \tau_{z}$.

Recall that there exists a unique $*$-anti-automorphism $R$ on $\mathcal{A}$ such that $\kappa=$ $R \tau_{-i / 2}=\tau_{-i / 2} R$ and $R^{2}=\mathrm{id}_{\mathcal{A}}$. This decomposition of $\kappa$ is called the polar decomposition of $\kappa ; R$ is called the unitary co-inverse of $\mathcal{A}$.

Note that $\psi(R \otimes R) \Delta=\Delta R$, where $\psi$ is the flip map. Also, $h R=h$. We refer the reader to section 7 of [15] for these results.

Lemma 7.1. Suppose that $\pi: \mathcal{A} \rightarrow \mathcal{A}$ is a surjective linear map. If $\pi$ is multiplicative (respectively, anti-multiplicative) and $h \pi=h$, then $\pi \rho_{z}=\rho_{z} \pi$ (respectively, $\left.\pi \rho_{z}=\rho_{-z} \pi\right)$, for all $z \in \mathbf{C}$.

Proof. We prove only the multiplicative case - the anti-multiplicative case is proved similarly. For $a, b \in \mathcal{A}$, we have $h\left(\rho_{i}(\pi(b)) \pi(a)\right)=h(\pi(a) \pi(b))=h(a b)=h\left(\rho_{i}(b) a\right)$ $=h\left(\pi\left(\rho_{i}(b)\right) \pi(a)\right)$. Therefore, surjectivity of $\pi$ and faithfulness of $h$ imply that $\rho_{i}(\pi(b))=\pi\left(\rho_{i}(b)\right)$. Hence, Lemma 9.2 implies that $\rho_{z} \pi=\pi \rho_{z}$, for all $z \in \mathbf{C}$.

The following special cases should be noted: $\tau_{z} \rho_{y}=\rho_{y} \tau_{z}, \rho_{y} R=R \rho_{-y}$ and $\rho_{y} \kappa=\kappa \rho_{-y}$, for all $y, z \in \mathbf{C}$. Note also that $\tau_{z} R=R \tau_{z}$ and $\tau_{z} \kappa=\kappa \tau_{z}$.

Lemma 7.2. Suppose that $\phi: \mathcal{A} \rightarrow \mathcal{A}$ is a surjective algebra homomorphism such that $h \phi=h$ and such that $h\left(a^{*} \phi(a)\right)$ is real, for all $a \in A$. Then $\phi\left(\phi\left(a^{*}\right)^{*}\right)=a$, for all $a \in \mathcal{A}$.

Proof. The function $\mathcal{A} \times \mathcal{A} \rightarrow \mathbf{C}:(a, b) \mapsto h\left(b^{*} \phi(a)\right)$ is a hermitian form on $\mathcal{A}$. Hence, for $a, b \in \mathcal{A}, h\left(\phi\left(\phi\left(a^{*}\right)^{*}\right) \phi(b)\right)=h\left(\phi\left(a^{*}\right)^{*} b\right)=h\left(b^{*} \phi\left(a^{*}\right)\right)^{-}=h(a \phi(b))$. Therefore, by faithfulness of $h, \phi\left(\phi\left(a^{*}\right)^{*}\right)=a$. 
Let $\int$ denote the integral on $\Omega$ associated to $\theta$. It is a twisted graded trace on $(\Omega, d)$, by [7, Corollary 4.9]. This means there is an algebra automorphism $\sigma$ of $\Omega$ of degree zero such $\sigma d=d \sigma$ and $\int \omega^{\prime} \omega=(-1)^{k l} \int \sigma(\omega) \omega^{\prime}$, for all non-negative integers $k$ and $l$ and all $\omega \in \Omega_{k}$ and $\omega^{\prime} \in \Omega_{l}$. Faithfulness of $\int$ implies that $\sigma$ is unique. We call $\sigma$ the twist automorphism of $\int$. Note that $\int \sigma=\int$. Also, $\sigma\left(\sigma\left(\omega^{*}\right)^{*}\right)=\omega$, for all $\omega \in \Omega$. In order to prove this we may and will assume that $\omega \in \Omega_{k}$. Since $\int$ is self adjoint, we get for all $\eta \in \Omega_{N-k}$,

$$
\begin{aligned}
\int \eta \sigma\left(\sigma(\omega)^{*}\right)^{*} & =(-1)^{k(N-k)}\left(\int \sigma\left(\sigma(\omega)^{*}\right) \eta^{*}\right)^{-} \\
& =\left(\int \eta^{*} \sigma(\omega)^{*}\right)^{-}=(-1)^{k(N-k)} \int \sigma(\omega) \eta=\int \eta \omega .
\end{aligned}
$$

Therefore the faithfulness of $\int$ implies that $\sigma\left(\sigma\left(\omega^{*}\right)^{*}\right)=\omega$. The restriction of $\sigma$ to $\mathcal{A}$ will be denoted by $\sigma_{\mathcal{A}}$; it is an automorphism on $\mathcal{A}$.

The proof of the following lemma is modeled on that of [12, Proposition 3.14].

Lemma 7.3. $\left(\kappa^{-2} \otimes \sigma\right) \Delta_{\Omega}=\Delta_{\Omega} \sigma$ and $\sigma\left(\Omega^{\text {inv }}\right)=\Omega^{\text {inv }}$.

Proof. Let $\omega \in \Omega_{k}$ and $\eta \in \Omega_{N-k}$. By [7, Theorem 4.4], we have

$$
\begin{aligned}
& \left(\operatorname{id}_{\mathcal{A}} \otimes \int\right)\left(\left(\kappa^{-2} \otimes \sigma\right)\left(\Delta_{\Omega}(\omega)\right)(1 \otimes \eta)\right) \\
& =\kappa^{-2}\left(\left(\operatorname{id}_{\mathcal{A}} \otimes \int\right)\left(\left(\operatorname{id}_{\mathcal{A}} \otimes \sigma\right)\left(\Delta_{\Omega}(\omega)\right)(1 \otimes \eta)\right)\right) \\
& =(-1)^{k(N-k)} \kappa^{-2}\left(\left(\operatorname{id}_{\mathcal{A}} \otimes \int\right)\left((1 \otimes \eta) \Delta_{\Omega}(\omega)\right)\right) \\
& =(-1)^{k(N-k)} \kappa^{-1}\left(\left(\operatorname{id}_{\mathcal{A}} \otimes \int\right)\left(\Delta_{\Omega}(\eta)(1 \otimes \omega)\right)\right) \\
& =\kappa^{-1}\left(\left(\operatorname{id}_{\mathcal{A}} \otimes \int\right)\left((1 \otimes \sigma(\omega)) \Delta_{\Omega}(\eta)\right)\right)=\left(\operatorname{id}_{\mathcal{A}} \otimes \int\right)\left(\Delta_{\Omega}(\sigma(\omega))(1 \otimes \eta)\right) .
\end{aligned}
$$

Consequently, faithfulness of $\int$ implies that $\left(\kappa^{-2} \otimes \sigma\right) \Delta_{\Omega}(\omega)=\Delta_{\Omega}(\sigma(\omega))$. This equation clearly implies in turn that $\sigma\left(\Omega^{\text {inv }}\right) \subseteq \Omega^{\text {inv }}$. Since $\sigma\left(\sigma\left(\omega^{*}\right)^{*}\right)=\omega$, we have $\sigma\left(\Omega^{\text {inv }}\right)=\Omega^{\text {inv }}$.

Theorem 7.4. For all $z \in \mathbf{C}, h \sigma_{\mathcal{A}}=h, \sigma_{\mathcal{A}} \rho_{z}=\rho_{z} \sigma_{\mathcal{A}}$ and $\sigma_{\mathcal{A}} \tau_{z}=\tau_{z} \sigma_{\mathcal{A}}$.

Proof. Theorem 4.5 of [7] implies that $h(\sigma(a))=\int \sigma(a) \theta=\int \theta a=h(a)$, for every $a \in \mathcal{A}$, proving that the first equation holds. The second equation now follows from Lemma 7.1

Using Lemma 7.3, we see that, for $z \in \mathbf{C}$,

$$
\begin{aligned}
& \left(\rho_{z} \kappa^{-2} \otimes \tau_{-z} \sigma_{\mathcal{A}}\right) \Delta=\left(\rho_{z} \otimes \tau_{-z}\right) \Delta \sigma_{\mathcal{A}}=\Delta \rho_{z} \sigma_{\mathcal{A}}=\Delta \sigma_{\mathcal{A}} \rho_{z} \\
& \quad=\left(\kappa^{-2} \otimes \sigma_{\mathcal{A}}\right) \Delta \rho_{z}=\left(\kappa^{-2} \rho_{z} \otimes \sigma_{\mathcal{A}} \tau_{-z}\right) \Delta=\left(\rho_{z} \kappa^{-2} \otimes \sigma_{\mathcal{A}} \tau_{-z}\right) \Delta,
\end{aligned}
$$

implying that $\left(\operatorname{id}_{\mathcal{A}} \otimes \tau_{-z} \sigma_{\mathcal{A}}\right) \Delta=\left(\operatorname{id}_{\mathcal{A}} \otimes \sigma_{\mathcal{A}} \tau_{-z}\right) \Delta$. Since $\mathcal{A}$ is the linear span of elements of the form $\left(f \otimes \operatorname{id}_{\mathcal{A}}\right) \Delta(a)$, where $a \in \mathcal{A}$ and $f \in \mathcal{A}^{\prime}$, we conclude that $\tau_{-z} \sigma_{\mathcal{A}}=\sigma_{\mathcal{A}} \tau_{-z}$.

We extend the concept of a one-parameter group from a $*$-algebra to our differential calculus $\Omega$. A map $\beta$ from $\mathbf{C}$ into the space of algebra automorphisms on $\Omega$ is a one-parameter group of automorphisms if $\beta_{y+z}=\beta_{y} \beta_{z}$ and $\beta_{z}(\omega)^{*}=\beta_{\bar{z}}\left(\omega^{*}\right)$, for 
all $y, z \in \mathbf{C}$ and $\omega \in \Omega$; furthermore, it is exponential if the map $\mathbf{C} \rightarrow \Omega: z \mapsto \beta_{z}(\omega)$ is of finite exponential type, for all $\omega \in \Omega$.

We shall call a family of elements $\left(e_{j}\right)_{j \in J}$ in $\mathcal{A}$ homogeneous if each of the elements $e_{j}$ belongs to some space $\mathcal{A}_{\alpha}$, for some $\alpha \in \hat{G}$.

Theorem 7.5. Suppose that $h\left(a^{*} \sigma(a)\right) \geq 0$, for all $a \in \mathcal{A}$. Then there exists a unique exponential one-parameter group $\beta$ on $\Omega$ such that $\beta_{i}=\sigma$. Moreover, there is a homogeneous orthonormal basis $\left(e_{j}\right)_{j \in J}$ for $\mathcal{A}$ and there are positive numbers $\left(\lambda_{j}\right)_{j \in J}$ such that $\beta_{z}\left(e_{j}\right)=\lambda_{j}^{-i z} e_{j}$, for all $j \in J$ and $z \in \mathbf{C}$.

Also, $\beta_{z}$ is of degree zero, $d \beta_{z}=\beta_{z} d$ and $\int \beta_{z}=\int$. Finally, $\left(\tau_{z} \otimes \beta_{z}\right) \Delta_{\Omega}=$ $\Delta_{\Omega} \beta_{z}$ and $\beta_{z}\left(\Omega^{\text {inv }}\right)=\Omega^{\text {inv }}$.

Proof. Uniqueness of $\beta$ is obvious, so we concern ourselves only with existence. If $\alpha \in \hat{G}, \kappa^{-2}\left(\mathcal{A}_{\alpha}\right)=\tau_{i}\left(\mathcal{A}_{\alpha}\right)=\mathcal{A}_{\alpha}$. Using Lemma 7.3, we get, for $i, j=1, \ldots, N_{\alpha}$,

$$
\Delta\left(\sigma\left(U_{i j}^{\alpha}\right)\right)=\left(\kappa^{-2} \otimes \sigma_{\mathcal{A}}\right) \Delta\left(U_{i j}^{\alpha}\right)=\sum_{k=1}^{N_{\alpha}} \kappa^{-2}\left(U_{i k}^{\alpha}\right) \otimes \sigma\left(U_{k j}^{\alpha}\right) .
$$

Applying $\operatorname{id}_{\mathcal{A}} \otimes \varepsilon$ to this equation, we get that the element $\sigma\left(U_{i j}^{\alpha}\right)$ is equal to $\sum_{k=1}^{N_{\alpha}} \kappa^{-2}\left(U_{i k}^{\alpha}\right) \varepsilon\left(\sigma\left(U_{k j}^{\alpha}\right)\right) \in \mathcal{A}_{\alpha}$. Hence, $\sigma\left(\mathcal{A}_{\alpha}\right) \subseteq \mathcal{A}_{\alpha}$. Define the bijective linear operator $P_{\alpha}$ on $\mathcal{A}_{\alpha}$ by setting $P_{\alpha}(a)=\sigma(a)$, for all $a \in \mathcal{A}_{\alpha}$. By assumption, $\left(P_{\alpha} a \mid a\right)=h\left(a^{*} \sigma(a)\right) \geq 0$, for all $a \in A_{\alpha}$; hence, $P_{\alpha}$ is positive. This allows us to define complex powers of $P_{\alpha}$.

Now define the function $\gamma$ from $\mathbf{C}$ into the space of linear maps on $\mathcal{A}$ such that for $z \in \mathbf{C}$ and $\alpha \in \hat{G}$, we have that $\gamma_{z}\left(\mathcal{A}_{\alpha}\right) \subseteq \mathcal{A}_{\alpha}$ and $\gamma_{z}(a)=P_{\alpha}^{-i z} a$, for all $a \in \mathcal{A}_{\alpha}$. It is clear that $\gamma_{n i}=\sigma_{\mathcal{A}}^{n}$, for all $n \in \mathbf{N}_{0}$.

By diagonalising each operator $P_{\alpha}$, we can find a homogeneous orthonormal basis $\left(e_{j}\right)_{j \in J}$ for $\mathcal{A}$ and positive numbers $\left(\lambda_{j}\right)_{j \in J}$ such that $\sigma\left(e_{j}\right)=\lambda_{j} e_{j}$, for all $j \in J$. Then $\gamma_{z}\left(e_{j}\right)=\lambda_{j}^{-i z} e_{j}$, for all $j \in J$ and $z \in \mathbf{C}$. Hence, Theorem 9.1 implies that $\sigma_{\mathcal{A}}$ is induced by an exponential one-parameter group, which must be equal to $\gamma$.

Let $z \in \mathbf{C}$. We will extend $\gamma_{z}$ to $\Omega$. Let $m$ and $n$ be a non-negative and a positive integer, respectively, and let $v_{j k}$ be elements in $\mathcal{A}$, where $j=1, \ldots, m$ and $k=0, \ldots, n$, such that $\sum_{j=1}^{m} v_{j 0} d v_{j 1} \cdots d v_{j n}=0$. Define the map $f: \mathbf{C} \rightarrow \Omega$ by setting $f(y)=\sum_{j=1}^{m} \gamma_{y}\left(v_{j 0}\right) d\left(\gamma_{y}\left(v_{j 1}\right)\right) \cdots d\left(\gamma_{y}\left(v_{j n}\right)\right)$, for all $y \in \mathbf{C}$. The function $f$ is clearly of finite exponential type. Moreover, for $l \in \mathbf{Z}$, we have

$$
\begin{aligned}
f(l i) & =\sum_{j=1}^{m} \gamma_{l i}\left(v_{j 0}\right) d\left(\gamma_{l i}\left(v_{j 1}\right)\right) \cdots d\left(\gamma_{l i}\left(v_{j n}\right)\right) \\
& =\sum_{j=1}^{m} \sigma^{l}\left(v_{j 0}\right) d\left(\sigma^{l}\left(v_{j 1}\right)\right) \cdots d\left(\sigma^{l}\left(v_{j n}\right)\right)=\sigma^{l}\left(\sum_{j=1}^{m} v_{j 0} d v_{j 1} \cdots d v_{j n}\right)=0 .
\end{aligned}
$$

Hence $f=0$. In particular, $f(z)=\sum_{j=1}^{m} \gamma_{z}\left(v_{j 0}\right) d\left(\gamma_{z}\left(v_{j 1}\right)\right) \cdots d\left(\gamma_{z}\left(v_{j n}\right)\right)=0$.

This allows us to define a map $\beta$ from $\mathbf{C}$ into the space of linear maps on $\Omega$ such that each $\beta_{z}$ is of degree zero and

$$
\beta_{z}\left(v_{0} d v_{1} \cdots d v_{n}\right)=\gamma_{z}\left(v_{0}\right) d\left(\gamma_{z}\left(v_{1}\right)\right) \cdots d\left(\gamma_{z}\left(v_{n}\right)\right),
$$

for all $z \in \mathbf{C}, n \in \mathbf{N}_{0}$ and $v_{0}, \ldots, v_{n} \in \mathcal{A}$. Hence, each $\beta_{z}$ is an algebra homomorphism extending $\gamma_{z}$ and $\beta_{z} d=d \beta_{z}$. The equality $\gamma_{z}(a)^{*}=\gamma_{\bar{z}}\left(a^{*}\right)$, for all $a \in \mathcal{A}$, extends easily to the equality $\beta_{z}(\omega)^{*}=\beta_{\bar{z}}\left(\omega^{*}\right)$, for all $\omega \in \Omega$. 
Furthermore, because $\gamma_{y} \gamma_{z}=\gamma_{y+z}$, we get $\beta_{y} \beta_{z}=\beta_{y+z}$, for all $y, z \in \mathbf{C}$. Therefore, $\beta$ is a one-parameter group on $\Omega$. Combining Equation (6) with the fact that $\gamma$ is an exponential one-parameter group on $\mathcal{A}$ shows that $\beta$ is exponential. Since $\int \sigma=\int$, the remarks preceding Theorem 9.1 imply that $\int \beta_{z}=\int$.

If $\omega \in \Omega$, the functions $z \mapsto\left(\tau_{z} \otimes \beta_{z}\right) \Delta_{\Omega}(\omega)$ and $z \mapsto \Delta_{\Omega}\left(\beta_{z}(\omega)\right)$ from $\mathbf{C}$ to $\mathcal{A} \otimes \Omega$ are of finite exponential type. For $n \in \mathbf{N}_{0}$, we have $\left(\tau_{n i} \otimes \beta_{n i}\right) \Delta_{\Omega}(\omega)=$ $\left(\kappa^{-2 n} \otimes \sigma^{n}\right) \Delta_{\Omega}(\omega)=\Delta_{\Omega}\left(\sigma^{n}(\omega)\right)=\Delta_{\Omega}\left(\beta_{n i}(\omega)\right)$. Therefore, the two functions agree on the whole complex plane. We conclude that $\left(\tau_{z} \otimes \beta_{z}\right) \Delta_{\Omega}=\Delta_{\Omega} \beta_{z}$, for all $z \in \mathbf{C}$. Clearly, this equality implies that $\beta_{z}\left(\Omega^{\text {inv }}\right)=\Omega^{\text {inv }}$, for all $z \in \mathbf{C}$.

The condition $h\left(a^{*} \sigma(a)\right) \geq 0$ is not easy to check. However, in the next result we give some equivalent conditions, of which the third one can be easily checked on an algebraic level.

Corollary 7.6. The following conditions are equivalent:

1. $h\left(a^{*} \sigma(a)\right) \geq 0$, for all $a \in \mathcal{A}$.

2. There exists an algebra automorphism $\phi$ on $\mathcal{A}$ such that $\phi^{2}=\sigma_{\mathcal{A}}, h \phi=h$ and $\phi\left(\phi\left(a^{*}\right)^{*}\right)=a$, for all $a \in \mathcal{A}$.

3. There exists an algebra automorphism $\phi$ on $\mathcal{A}$ such that $\phi^{2}=\sigma_{\mathcal{A}}$, $\left(\tau_{i / 2} \otimes \phi\right) \Delta=\Delta \phi$ and $\phi\left(\phi\left(a^{*}\right)^{*}\right)=a$, for all $a \in \mathcal{A}$.

Proof. First we show $1 \Rightarrow 3$. Therefore, assume Condition 1 . By the theorem, there exists a unique exponential one-parameter group $\beta$ on $\mathcal{A}$ such that $\beta_{i}=\sigma_{\mathcal{A}}$. Put $\phi=\beta_{i / 2}$. Then $\phi$ satisfies the conditions in Condition 3 .

The implication $3 \Rightarrow 2$ follows from fact that the equality $\left(\tau_{i / 2} \otimes \phi\right) \Delta=\Delta \phi$ implies $h \phi=h$.

Finally, we show the implication $2 \Rightarrow 1$ holds: Assuming Condition 2, we get, for $a \in \mathcal{A}, h\left(a^{*} \sigma(a)\right)=h\left(a^{*} \phi^{2}(a)\right)=h\left(\phi^{-1}\left(a^{*} \phi^{2}(a)\right)\right)=h\left(\phi^{-1}\left(a^{*}\right) \phi(a)\right)=$ $h\left(\phi(a)^{*} \phi(a)\right)$

$\geq 0$.

It is useful to note that, of course, the equations in Condition 3 of the corollary hold for all $a$ in $\mathcal{A}$ if they hold for all $a$ in a set of algebra generators of $\mathcal{A}$.

Suppose that $h\left(a^{*} \sigma(a)\right) \geq 0$, for all $a \in \mathcal{A}$. Let $\beta$ denote the unique exponential one-parameter group on $\Omega$ such that $\beta_{i}=\sigma$. We denote by $\gamma$ and $\gamma^{\prime}$ the exponential one-parameter groups on $\mathcal{A}$ obtained, respectively, by restricting $\beta$ and by setting $\gamma_{z}^{\prime}=R \gamma_{z} R$, for all $z \in \mathbf{C}$.

Since $\sigma_{\mathcal{A}} \rho_{z}=\rho_{z} \sigma_{\mathcal{A}}$ and $\sigma_{\mathcal{A}} \tau_{z}=\tau_{z} \sigma_{\mathcal{A}}$, for all $z \in \mathbf{C}$, Lemma 9.2 implies that $\gamma_{y} \rho_{z}=\rho_{z} \gamma_{y}$ and $\gamma_{y} \tau_{z}=\tau_{z} \gamma_{y}$, for all $y, z \in \mathbf{C}$. Because $\kappa=R \tau_{-i / 2}=\tau_{-i / 2} R$, we get $\gamma_{z}^{\prime}=\kappa \gamma_{z} \kappa^{-1}=\kappa^{-1} \gamma_{z} \kappa$. Also, $\gamma_{y}^{\prime} \rho_{z}=\rho_{z} \gamma_{y}^{\prime}$ and $\gamma_{y}^{\prime} \tau_{z}=\tau_{z} \gamma_{y}^{\prime}$, since $R \rho_{z}=\rho_{-z} R$ and $R \tau_{z}=\tau_{z} R$.

Lemma 7.7. Suppose that $h\left(a^{*} \sigma(a)\right) \geq 0$, for all $a \in \mathcal{A}$. Let $y, z \in \mathbf{C}$. Then:

1. $\left(\gamma_{z}^{\prime} \otimes \tau_{z}\right) \Delta=\Delta \gamma_{z}^{\prime}$.

2. $\left(\gamma_{z}^{\prime} \otimes \gamma_{z}\right) \Delta=\Delta \gamma_{z}^{\prime} \gamma_{z} \tau_{-z}$.

3. $\gamma_{z}^{\prime} \gamma_{y}=\gamma_{y} \gamma_{z}^{\prime}$.

Proof. Theorem 7.5 implies that $\left(\tau_{z} \otimes \gamma_{z}\right) \Delta=\Delta \gamma_{z}$. Hence, if $\psi$ is the flip map,

$$
\begin{aligned}
& \left(\gamma_{z}^{\prime} \otimes \tau_{z}\right) \Delta=\left(\kappa \gamma_{z} \kappa^{-1} \otimes \kappa \tau_{z} \kappa^{-1}\right) \Delta=\left(\kappa \gamma_{z} \otimes \kappa \tau_{z}\right) \psi \Delta \kappa^{-1} \\
& \quad=\psi\left(\kappa \tau_{z} \otimes \kappa \gamma_{z}\right) \Delta \kappa^{-1}=\psi(\kappa \otimes \kappa) \Delta \gamma_{z} \kappa^{-1}=\Delta \kappa \gamma_{z} \kappa^{-1}=\Delta \gamma_{z}^{\prime},
\end{aligned}
$$


and therefore,

$$
\begin{gathered}
\left(\gamma_{z}^{\prime} \otimes \gamma_{z}\right) \Delta=\left(\gamma_{z}^{\prime} \tau_{z} \otimes \gamma_{z} \tau_{z}\right) \Delta \tau_{-z}=\left(\gamma_{z}^{\prime} \tau_{z} \otimes \tau_{z} \gamma_{z}\right) \Delta \tau_{-z} \\
=\left(\gamma_{z}^{\prime} \otimes \tau_{z}\right) \Delta \gamma_{z} \tau_{-z}=\Delta \gamma_{z}^{\prime} \gamma_{z} \tau_{-z}
\end{gathered}
$$

Also,

$$
\begin{aligned}
\Delta \gamma_{z}^{\prime} \gamma_{y}=\left(\gamma_{z}^{\prime} \otimes \tau_{z}\right) \Delta \gamma_{y} & =\left(\gamma_{z}^{\prime} \tau_{y} \otimes \tau_{z} \gamma_{y}\right) \Delta \\
=\left(\tau_{y} \gamma_{z}^{\prime} \otimes \gamma_{y} \tau_{z}\right) \Delta & =\left(\tau_{y} \otimes \gamma_{y}\right) \Delta \gamma_{z}^{\prime}=\Delta \gamma_{y} \gamma_{z}^{\prime} .
\end{aligned}
$$

Therefore, injectivity of $\Delta$ implies that $\gamma_{z}^{\prime} \gamma_{y}=\gamma_{y} \gamma_{z}^{\prime}$.

Lemma 7.8. Suppose that $h\left(a^{*} \sigma(a)\right) \geq 0$, for all $a \in \mathcal{A}$. For every integer $k \in \mathbf{N}_{0}$, there exists a basis $\left(\omega_{k n}\right)_{n=1}^{m_{k}}$ for $\Omega_{k}^{\text {inv }}$ and positive numbers $\left(\nu_{k n}\right)_{n=1}^{m_{k}}$ such that $\beta_{z}\left(\omega_{k n}\right)=\nu_{k n}^{-i z} \omega_{k n}$, for all $n=1, \ldots, m_{k}$ and $z \in \mathbf{C}$.

Proof. Define the linear map $w: \mathcal{A} \rightarrow \Omega_{1}$ by setting $w(a)=m(\kappa \otimes d) \Delta(a)$, for all $a \in \mathcal{A}$. Here $m: \mathcal{A} \otimes \Omega_{1} \rightarrow \Omega_{1}$ is the linear map given by $m(a \otimes \omega)=a \omega$, for all $a \in \mathcal{A}$ and $\omega \in \Omega_{1}$. Observe that $w(\mathcal{A})=\Omega_{1}^{\text {inv }}$. We refer the reader to [5, Section 14.1.1] for more information on the map $w$.

By Lemma 7.7 we have, for all $a \in \mathcal{A}$,

$$
\begin{aligned}
\beta_{z}(w(a)) & =\beta_{z}(m(\kappa \otimes d) \Delta(a))=m\left(\gamma_{z} \kappa \otimes \beta_{z} d\right) \Delta(a)=m\left(\kappa \gamma_{z}^{\prime} \otimes d \gamma_{z}\right) \Delta(a) \\
& =m(\kappa \otimes d) \Delta\left(\left(\gamma_{z}^{\prime} \gamma_{z} \tau_{-z}\right)(a)\right)=w\left(\left(\gamma_{z}^{\prime} \gamma_{z} \tau_{-z}\right)(a)\right) .
\end{aligned}
$$

Define the automorphism $\phi$ on $\mathcal{A}$ by setting $\phi=\gamma_{i / 2}^{\prime} \gamma_{i / 2} \tau_{-i / 2}$. Then $\phi^{2}=$ $\gamma_{i}^{\prime} \gamma_{i} \tau_{-i}, h \phi=h$ and $\phi\left(\phi\left(a^{*}\right)^{*}\right)=a$, for all $a \in \mathcal{A}$. As in the proof of Corollary 7.6, this implies that $h\left(a^{*} \phi^{2}(a)\right) \geq 0$, for all $a \in \mathcal{A}$.

Let $\alpha \in \hat{G}$. In the same way as in the beginning of the proof of Theorem 7.5 , the equalities $\Delta \gamma_{i / 2}^{\prime}=\left(\gamma_{i / 2}^{\prime} \otimes \tau_{i / 2}\right) \Delta$ and $\tau_{i / 2}\left(\mathcal{A}_{\alpha}\right)=\mathcal{A}_{\alpha}$ allow us to conclude that $\gamma_{i / 2}^{\prime}\left(\mathcal{A}_{\alpha}\right)=\mathcal{A}_{\alpha}$. Similarly, $\gamma_{i / 2}\left(\mathcal{A}_{\alpha}\right)=\mathcal{A}_{\alpha}$. Hence, $\phi\left(\mathcal{A}_{\alpha}\right)=\mathcal{A}_{\alpha}$, for all $\alpha \in \hat{G}$. We can therefore use the same idea as in the proof of Theorem 7.5 to find a basis $\left(f_{j}\right)_{j \in J}$ of $\mathcal{A}$ and positive numbers $\left(\mu_{j}\right)_{j \in J}$ such that $\phi^{2}\left(f_{j}\right)=\mu_{j} f_{j}$, for all $j \in J$.

Equation (7) gives $\beta_{i}\left(w\left(f_{j}\right)\right)=\mu_{j} w\left(f_{j}\right)$, for all $j \in J$. Hence, $\beta_{l i}\left(w\left(f_{j}\right)\right)=$ $\left(\beta_{i}\right)^{l}\left(w\left(f_{j}\right)\right)=\mu_{j}^{l} w\left(f_{j}\right)$, for all $l \in \mathbf{N}_{0}$. The functions $z \mapsto \beta_{z}\left(w\left(f_{j}\right)\right)$ and $z \mapsto$ $\mu_{j}^{-i z} w\left(f_{j}\right)$ from $\mathbf{C}$ to $\Omega_{1}$ are both of finite exponential type and agree on $\mathbf{N}_{0} i$, implying that they are equal on the whole complex plane. Thus, $\beta_{z}\left(w\left(f_{j}\right)\right)=$ $\mu_{j}^{-i z} w\left(f_{j}\right)$, for all $z \in \mathbf{C}$.

The lemma now follows easily, using the fact that $\Omega_{k}^{\text {inv }}$ is the linear span of the elements $w\left(f_{j_{1}}\right) \cdots w\left(f_{j_{k}}\right)$, where $j_{1}, \ldots, j_{k} \in J$, combined with the fact that, for $z \in$ $\mathbf{C}$, the multiplicativity of $\beta_{z}$ implies that $\beta_{z}\left(w\left(f_{j_{1}}\right) \cdots w\left(f_{j_{k}}\right)\right)=\left(\mu_{j_{1}} \cdots \mu_{j_{k}}\right)^{-i z}$ $w\left(f_{j_{1}}\right) \cdots w\left(f_{j_{k}}\right)$, for all $j_{1}, \ldots, j_{k} \in J$.

By defining the inner-product on each space $\Omega_{k}^{\text {inv }}$ in such a way that the basis in the previous lemma is orthonormal, we get the following theorem.

Theorem 7.9. There exists a graded, left-invariant inner product $(\cdot \mid \cdot)$ on $\Omega$ such that $(\sigma(\omega) \mid \omega) \geq 0$, for all $\omega \in \Omega$, if, and only if, $h\left(a^{*} \sigma(a)\right) \geq 0$, for all $a \in \mathcal{A}$.

Theorem 7.10. Suppose we are given a graded, left-invariant inner product $(\cdot \mid \cdot)$ on $\Omega$ relative to which $\sigma$ is positive. Let $\beta$ be the unique exponential one-parameter group on $\Omega$ such that $\beta_{i}=\sigma$. Then the following conditions hold:

1. $\left(\beta_{z}(\omega) \mid \omega\right) \geq 0$, for all $\omega \in \Omega$ and $z \in \mathbf{R} i$. 
2. $\left(\beta_{z}(\omega) \mid \eta\right)=\left(\omega \mid \beta_{-\bar{z}}(\eta)\right)$, for all $\omega, \eta \in \Omega$.

3. $d^{*} \beta_{z}=\beta_{z} d^{*}, D \beta_{z}=\beta_{z} D$ and $\nabla \beta_{z}=\beta_{z} \nabla$, for all $z \in \mathbf{C}$.

4. For $L$ the Hodge operator, we have $L \beta_{z}=\beta_{z} L$, for all $z \in \mathbf{C}$.

Proof. Combining Theorem 7.5 and Lemma 7.8 shows that there exists an orthonormal basis $\left(\eta_{k}\right)_{k \in K}$ of $\Omega$ and a family of positive numbers $\left(\nu_{k}\right)_{k \in K}$ such that $\beta_{z}\left(\eta_{k}\right)=\nu_{k}^{-i z} \eta_{k}$, for all $k \in K$. From this, Conditions 1 and 2 follow. Condition 2 implies that, if $z \in \mathbf{C}$, then $\beta_{-\bar{z}}$ is adjointable and that its adjoint is $\beta_{z}$. Hence, taking the adjoint of the equation $d \beta_{-\bar{z}}=\beta_{-\bar{z}} d$, we get $\beta_{z} d^{*}=d^{*} \beta_{z}$. The other two equalities of Condition 3 follow immediately.

If $\omega, \eta \in \Omega$, then, using the fact that $\int \sigma=\int$, we have

$$
\begin{aligned}
\int \omega^{*} L(\sigma(\eta)) & =(\sigma(\eta) \mid \omega)=(\eta \mid \sigma(\omega))=\int \sigma(\omega)^{*} L(\eta) \\
& =\int \sigma\left(\sigma(\omega)^{*}\right) \sigma(L(\eta))=\int \omega^{*} \sigma(L(\eta)) .
\end{aligned}
$$

Hence, by faithfulness of $\int, L(\sigma(\eta))=\sigma(L(\eta))$ and therefore, by Lemma 9.2 (more precisely, by a result for $\Omega$ that is analogous), $L \beta_{z}=\beta_{z} L$, for all $z \in \mathbf{C}$. Thus, Condition 4 holds.

Theorem 7.11. Suppose that $h\left(a^{*} \sigma(a)\right) \geq 0$, for all $a \in \mathcal{A}$. Then there exists a graded, left-invariant inner product $(\cdot \mid \cdot)$ on $\Omega$ such that $(\sigma(\omega) \mid \omega) \geq 0$, for all $\omega \in \Omega$, and such that the Hodge operator $L$ associated to this inner product satisfies the conditions of Theorem 6.1.

Proof. First we choose, as we may, a graded, left-invariant inner product $(\cdot \mid \cdot)^{\prime}$ on $\Omega$ such that $(\sigma(\omega) \mid \omega)^{\prime} \geq 0$, for all $\omega \in \Omega$. Now construct a new graded, left-invariant inner product $(\cdot \mid \cdot)$ by the method outlined in Section 6. One easily checks that $(\sigma(\omega) \mid \omega) \geq 0$, for all $\omega \in \Omega_{k}$, if $k \neq N / 2$, by using the fact that $\sigma$ and the Hodge operator $L^{\prime}$ associated to $(\cdot \mid \cdot)^{\prime}$ commute. We now show that by refining the method used in Section 6 to define the inner product on $\Omega_{k}$, when $k=N / 2$, we can arrange to have in this case also that $(\sigma(\omega) \mid \omega) \geq 0$, for all $\omega \in \Omega_{k}$.

Let $T$ be the unique linear operator on $\Omega_{k}^{\text {inv }}$ such that $(T \omega \mid \eta)^{\prime}=\int \eta^{*} \omega$, for all $\omega, \eta \in \Omega_{k}^{\text {inv }}$. Then $T^{*}=(-1)^{k(N-k)} T$. Using the fact that $\sigma$ is a positive operator with respect to $(\cdot \mid \cdot)^{\prime}$, and that, for $\omega, \eta \in \Omega_{k}^{\text {inv }}, \int \eta^{*} \sigma(\omega)=\int \sigma^{-1}\left(\eta^{*} \sigma(\omega)\right)=$ $\int \sigma(\eta)^{*} \omega$, it follows that the operators $\sigma$ and $T$ commute. Since these operators are normal, there is an orthogonal basis $u_{1}, \ldots, u_{m}$ of $\Omega_{k}^{\text {inv }}$ consisting of eigenvectors of $\sigma$ and $T$. Setting $\varepsilon_{k}=1$, if $(-1)^{k(N-k)}=1$ and $\varepsilon_{k}=i$, if $(-1)^{k(N-k)}=-1$, we can re-normalize the basis elements $u_{j}$ to get a new basis $e_{1}, \ldots, e_{m}$ of eigenvectors of $\sigma$ such that $\int e_{q}^{*} e_{p}= \pm \varepsilon_{k} \delta_{p q}$, for $p, q=1, \ldots, m$. We now proceed as before, in Section 6, to choose a new inner product on $\Omega_{k}^{\text {inv }}$ making the basis $e_{j}$ orthonormal. From this we complete our construction to get a graded, left-invariant inner product $(\cdot \mid \cdot)$ on $\Omega$. For this inner product the associated Hodge operator $L$ satisfies the conditions of Theorem 6.1. Also, $\sigma$ is clearly positive for this inner product.

\section{WORONOWICZ' 3-DIMENSIONAL CALCULUS}

In this section we compute the eigenvalues of the Laplacian for a special 3dimensional calculus over the quantum group $S U_{q}(2)$, where $q$ is a real number for 
which $0<|q|<1$. This calculus has a special interest, since it was the first calculus constructed by Woronowicz in the quantum group setting.

We begin by recalling some basic facts about the calculus (see 13] for details). It is a left-covariant, 3 -dimensional $*$-differential calculus $(\Omega, d)$ over the Hopf algebra $\mathcal{A}_{q}$ underlying $S U_{q}(2)$. The space $\Omega_{1}^{\text {inv }}$ has a linear basis $\eta_{1}, \eta_{2}, \eta_{3}$ such that $\eta_{1} \eta_{2}, \eta_{2} \eta_{3}, \eta_{3} \eta_{1}$ is a basis of $\Omega_{2}^{\text {inv }}$ and $\eta_{1} \eta_{2} \eta_{3}$ is one of $\Omega_{3}^{\text {inv }}$. The involution on $\Omega$ is determined by the relations

$$
\eta_{1}^{*}=q \eta_{3}, \eta_{2}^{*}=-\eta_{2}, \eta_{3}^{*}=q^{-1} \eta_{1} .
$$

Left-covariance implies that there exists linear functionals $\chi_{1}, \chi_{2}, \chi_{3}$ on $\mathcal{A}$ such that

$$
d a=\sum_{r=1}^{3}\left(\chi_{r} * a\right) \eta_{r},
$$

for all $a \in \mathcal{A}$. We note that

$$
\chi_{1}^{*}=-q^{-1} \chi_{3}, \chi_{2}^{*}=\chi_{2}, \chi_{3}^{*}=-q \chi_{1} .
$$

As usual, we shall set $\chi=\sum_{r=1}^{3} \chi_{r}^{*} \chi_{r}$. Note that $d\left(\Omega_{2}^{\text {inv }}\right)=0$, by Equation Set (3.13) of [13].

The following commutation relations will be needed frequently [13, Table 5]:

$$
\eta_{2} \eta_{1}=-q^{4} \eta_{1} \eta_{2}, \eta_{3} \eta_{2}=-q^{4} \eta_{2} \eta_{3}, \eta_{1} \eta_{3}=-q^{-2} \eta_{3} \eta_{1} .
$$

Note also that $\eta_{r}^{2}=0$, for $r=1,2,3$. It follows that $\eta_{r} \eta_{s} \eta_{t}=c \eta_{1} \eta_{2} \eta_{3}$, for some scalar $c$ and that $c \neq 0$ if, and only if, the subscripts $r, s, t$ are distinct.

We set $\theta=\eta_{1} \eta_{2} \eta_{3}$. Then $\theta^{*}=\theta$.

We now choose an inner product on $\Omega^{\text {inv }}$ such that the vectors

$$
1, \eta_{1}, \eta_{2}, \eta_{3}, \eta_{1} \eta_{2}, \eta_{2} \eta_{3}, \eta_{3} \eta_{1}, \eta_{1} \eta_{2} \eta_{3}
$$

form an orthonormal basis. In the usual way, this allows us to define a graded, left-invariant inner product on $\Omega$. Then $\theta$ is a volume element, since $\theta^{*}=\theta$ and $\|\theta\|=1$. We let $\int$ denote the integral associated to $\theta$.

If $\eta_{r} \eta_{s} \eta_{t}=c \eta_{1} \eta_{2} \eta_{3}$, then $c=\int \eta_{r} \eta_{s} \eta_{t}$; hence, $\int \eta_{r} \eta_{s} \eta_{t} \neq 0$ if, and only if, $r, s, t$ are distinct.

Theorem 8.1. $\Omega$ is non-degenerate.

Proof. We have to show that if $0 \leq k \leq 3$ and $\omega \in \Omega_{k}$ and $\omega^{\prime} \omega=0$, for all $\omega^{\prime} \in \Omega_{3-k}$, then $\omega=0$.

Consider first the case where $k=0$, so that $\omega=a$, for some element $a \in \mathcal{A}_{q}$. Then, we suppose $\theta b a=0$, for all $b \in \mathcal{A}_{q}$. If we take $b=1$, this becomes $\theta a=0$ and therefore, $a=0$, as required.

Now we consider the case where $k=1$. In this case we may write $\omega=\sum_{r=1}^{3} \eta_{r} a_{r}$, for some elements $a_{1}, a_{2}, a_{3} \in \mathcal{A}_{q}$. If $s, t$ are distinct elements of $\{1,2,3\}$, then $0=\eta_{s} \eta_{t} \omega=\eta_{s} \eta_{t} \eta_{r} a_{r}$, where $r, s, t$ are distinct. Hence, $a_{r}=0$. It follows that $\omega=0$.

Suppose now $k=2$. In this case, we may write $\omega=\eta_{1} \eta_{2} a_{1}+\eta_{2} \eta_{3} a_{2}+\eta_{3} \eta_{1} a_{3}$, for some elements $a_{1}, a_{2}, a_{3} \in \mathcal{A}_{q}$. Then $0=\eta_{3} \omega=\eta_{3} \eta_{1} \eta_{2} a_{1}$. Hence, $a_{1}=0$. Similarly, $a_{2}=0$ and $a_{3}=0$ and therefore, $\omega=0$.

If $k=3$, then $\omega=1 \omega=0$.

Thus, in all cases, $\omega=0$ and therefore $\Omega$ is non-degenerate, as required. 
It follows from non-degeneracy of $\Omega$ that $\int$ is faithful (Lemma 3.1) and a Hodge operator $L^{\prime}$ exists (Theorem 3.2). We need to know the values of $L^{\prime}$ on $\eta_{1}, \eta_{2}, \eta_{3}$. First, set $L^{\prime}\left(\eta_{1}\right)=\lambda_{1} \eta_{1} \eta_{2}+\lambda_{2} \eta_{2} \eta_{3}+\lambda_{3} \eta_{3} \eta_{1}$, where $\lambda_{1}, \lambda_{2}, \lambda_{3}$ are scalars to be determined. Observe that $\int \eta_{s}^{*} L^{\prime}\left(\eta_{r}\right)=\left(\eta_{r} \mid \eta_{s}\right)=\delta_{r s}$. Hence, $1=\int \eta_{1}^{*} L^{\prime}\left(\eta_{1}\right)=$ $\int q \eta_{3} \lambda_{1} \eta_{1} \eta_{2}=q \lambda_{1} \int\left(-q^{2}\right) \eta_{1} \eta_{3} \eta_{2}=-q^{3} \lambda_{1} \int \eta_{1}\left(-q^{4} \eta_{2} \eta_{3}\right)=q^{7} \lambda_{1} \int \eta_{1} \eta_{2} \eta_{3}=q^{7} \lambda_{1}$. Hence, $\lambda_{1}=q^{-7}$. Similar reasoning shows that $\lambda_{2}=0$ and $\lambda_{3}=0$. Continuing this way we can calculate that

$$
L^{\prime}\left(\eta_{1}\right)=q^{-7} \eta_{1} \eta_{2}, L^{\prime}\left(\eta_{2}\right)=-q^{-6} \eta_{3} \eta_{1}, L^{\prime}\left(\eta_{3}\right)=q \eta_{2} \eta_{3} .
$$

Now we redefine the inner product on $\Omega$ as in Section 6 to obtain a new Hodge operator $L$ satisfying the conditions of Theorem 6.1. The new inner product remains unchanged on $\Omega_{0}^{\text {inv }}, \Omega_{1}^{\text {inv }}$ and $\Omega_{3}^{\text {inv }}$ (since $L^{\prime}(1)=\theta$ ), but on $\Omega_{2}^{\text {inv }}$ it is chosen in such a way that $L$ maps $\Omega_{1}^{\text {inv }}$ isometrically onto $\Omega_{2}^{\text {inv }}$. Hence, for the new inner product, the elements

$$
1, \eta_{1}, \eta_{2}, \eta_{3}, q^{-7} \eta_{1} \eta_{2}, q \eta_{2} \eta_{3},-q^{-6} \eta_{3} \eta_{1}, \eta_{1} \eta_{2} \eta_{3}
$$

form an orthonormal basis.

Since $L=L^{\prime}$ on $\Omega_{1}^{\text {inv }}$ and since $L^{2}=1$ we get

$$
L\left(\eta_{1}\right)=q^{-7} \eta_{1} \eta_{2}, L\left(\eta_{2}\right)=-q^{-6} \eta_{3} \eta_{1}, L\left(\eta_{3}\right)=q \eta_{2} \eta_{3}
$$

and

$$
L\left(\eta_{1} \eta_{2}\right)=q^{7} \eta_{1}, L\left(\eta_{2} \eta_{3}\right)=q^{-1} \eta_{3}, L\left(\eta_{3} \eta_{1}\right)=-q^{6} \eta_{2} .
$$

Now set $E_{0}=\operatorname{id}_{\mathcal{A}_{q}}$ and $E_{r}=E_{\chi_{r}}$, for $r=1,2,3$. Also, denote by $M_{0}$ the restriction of $d$ to $\Omega^{\text {inv }}$ and, for $r=1,2,3$, denote by $M_{r}$ the operator on $\Omega^{\text {inv }}$ of left multiplication by $\eta_{r}$. Using the usual identification of $\Omega$ with $\mathcal{A}_{q} \otimes \Omega^{\text {inv }}$, we have $d=\sum_{r=0}^{3} E_{r} \otimes M_{r}$, by Equation (2). If $\ell$ denotes the restriction of $L$ to $\Omega^{\mathrm{inv}}$, then clearly $L=\operatorname{id}_{\mathcal{A}_{q}} \otimes \ell$. Hence $L d=\sum_{r=0}^{3} E_{r} \otimes \ell M_{r}$.

Now let $T$ be the restriction map $L d: \Omega_{1} \rightarrow \Omega_{1}$, and, for $r=0,1,2,3$, let $T_{r}$ be the restriction of $\ell M_{r}$ to $\Omega_{1}^{\text {inv }}$. Then $T=\sum_{r=0}^{3} E_{r} \otimes T_{r}$.

Observe that $T=T^{*}$ and that $T^{2}(\omega)=d^{*} d \omega$, for all $\omega \in \Omega_{1}$. For, if $\omega, \omega^{\prime} \in \Omega_{1}$, then $\left(\omega \mid T \omega^{\prime}\right)=\left(\omega \mid L d \omega^{\prime}\right)=\left(\omega \mid d^{*} L \omega^{\prime}\right)$, by Theorem 6.1. Hence, $\left(\omega \mid T \omega^{\prime}\right)=$ $\left(d \omega \mid L \omega^{\prime}\right)=\left(L d \omega \mid L^{2} \omega^{\prime}\right)=\left(T \omega \mid \omega^{\prime}\right)$, since $L^{2}=$ id. Hence, $T$ is adjointable, with adjoint $T^{*}=T$. Also, $T^{2}(\omega)=L d L d(\omega)=d^{*} L L d(\omega)=d^{*} d(\omega)$.

This identification of a self-adjoint square root $T$ for the restriction of $d^{*} d$ to $\Omega_{1}$ will be the key to our approach to calculating the eigenvalues of the Laplacian $\nabla$ on $\Omega_{1}$. In fact, we shall calculate the eigenvalues of $T$ and use these and some calculations from Section 5 to find the eigenvalues of $\nabla$ on $\Omega_{1}$.

It is straightforward to calculate the matrices of the operators $T_{0}, T_{1}, T_{2}, T_{3}$ (relative to the basis $\eta_{1}, \eta_{2}, \eta_{3}$ ), using the following Cartan-Maurer formulas from [13, Table 6]:

$$
d \eta_{1}=q^{2}\left(1+q^{2}\right) \eta_{1} \eta_{2}, d \eta_{2}=q \eta_{1} \eta_{3}, d \eta_{3}=q^{2}\left(1+q^{2}\right) \eta_{2} \eta_{3} .
$$

Hence, $L d \eta_{1}=q^{9}\left(1+q^{2}\right) \eta_{1}, L d \eta_{2}=q^{5} \eta_{2}$ and $L d \eta_{3}=q\left(1+q^{2}\right) \eta_{3}$. Therefore, the matrix form of $T_{0}$ is given by

$$
T_{0}=\left[\begin{array}{ccc}
q^{9}\left(1+q^{2}\right) & 0 & 0 \\
0 & q^{5} & 0 \\
0 & 0 & q\left(1+q^{2}\right)
\end{array}\right] .
$$


Since $T_{1}(\omega)=L\left(\eta_{1} \omega\right)$, we have $T_{1}\left(\eta_{1}\right)=0, T_{1}\left(\eta_{2}\right)=q^{7} \eta_{1}$ and $T_{1}\left(\eta_{3}\right)=q^{4} \eta_{2}$, which gives us the matrix of $T_{1}$. We can calculate $T_{2}$ and $T_{3}$ similarly. We get

$$
T_{1}=\left[\begin{array}{ccc}
0 & q^{7} & 0 \\
0 & 0 & q^{4} \\
0 & 0 & 0
\end{array}\right], T_{2}=\left[\begin{array}{ccc}
-q^{11} & 0 & 0 \\
0 & 0 & 0 \\
0 & 0 & q^{-1}
\end{array}\right], T_{3}=\left[\begin{array}{ccc}
0 & 0 & 0 \\
-q^{6} & 0 & 0 \\
0 & -q^{3} & 0
\end{array}\right] .
$$

Now, if $\eta_{r s}$ is the system of matrix units for $B\left(\Omega_{1}^{\text {inv }}\right)$ corresponding to the basis $\eta_{1}, \eta_{2}, \eta_{3}$, then $T=\sum_{r, s=1}^{3} T_{r s} \otimes \eta_{r s}$, where, from our calculations,

$$
\left[\begin{array}{lll}
T_{11} & T_{12} & T_{13} \\
T_{21} & T_{22} & T_{23} \\
T_{31} & T_{32} & T_{33}
\end{array}\right]=\left[\begin{array}{ccc}
q^{9}\left(1+q^{2}\right)-q^{11} E_{2} & q^{7} E_{1} & 0 \\
-q^{6} E_{3} & q^{5} & q^{4} E_{1} \\
0 & -q^{3} E_{3} & q\left(1+q^{2}\right)+q^{-1} E_{2}
\end{array}\right]
$$

It is useful to observe that, if $a \in \mathcal{A}_{q}$, then

$$
T\left(a \eta_{s}\right)=\sum_{r=1}^{3} T_{r s}(a) \eta_{r}
$$

The formula for $T$ we have derived is still not explicit enough to enable us to calculate eigenvalues. To make things fully explicit, we now need to use some aspects of the representation theory of $S U_{q}(2)$. Recall that this quantum group has a complete system of inequivalent invertible irreducible representations $W^{1}, W^{2}, \ldots, W^{M}, \ldots$, where $W^{M}$ acts on a Hilbert space $H_{M}$ of dimension $M$.

The $W^{M}$ are defined as in [13, Equation 5.32]. They are not unitary, which is not in line with our practice up to this point of only dealing with unitary representations. However, we shall be making extensive use of various formulas from [13, so we use the $W^{M}$. There is a standard way of constructing an equivalent unitary representation to a given invertible representation. If $Q_{M}=h\left(W^{M *} W^{M}\right)$ (that is, the scalar matrix obtained by applying $h$ to each entry of $W^{M *} W^{M}$ ), then $Q_{M}$ is positive and invertible and $U^{M}=Q_{M}^{1 / 2} W^{M} Q_{M}^{-1 / 2}$ is an irreducible unitary representation equivalent to $W^{M}$. See [14, Theorem 5.2] for details.

We shall need some quasi-orthogonality relations for the $W^{M}$. Since the matrix entries of $U^{M}$ are orthogonal to those of $U^{N}$, if $M \neq N$, the matrix entries of $W^{M}$ are orthogonal to those of $W^{N}$.

It is well known that $U_{p k}^{M}$ and $U_{t u}^{M}$ are orthogonal if $k \neq u$ [11, Theorem 7.3]. It turns out that the elements $W_{p k}^{M}$ have this property also, and this will be important in the sequel. The reason is that in this special case the matrix $Q_{M}$ is diagonal, so that there are positive numbers $q_{M}(k)$ such that $U_{p k}^{M}=q_{M}(p) q_{M}(k)^{-1} W_{p k}^{M}$.

For this example we even have that $h\left(\left(W_{p k}^{M}\right)^{*} W_{p^{\prime} l}^{M}\right)=0$ if $k \neq l$ or $p \neq p^{\prime}$. A proof of this fact will be given in Lemma 8.9 .

Fix $M \geq 1$. Set $A_{r}=\chi_{r}\left(W^{M}\right)$, for $r=1,2,3$ (these matrices are the infinitesimal generators of the representation $W^{M}$; see [13. Theorem 5.4]). Let $m$ be the integer or half-integer for which $M=2 m+1$. Then the space $H_{M}$ admits an orthogonal basis $\xi_{-m}, \xi_{-m+1}, \ldots, \xi_{m-1}, \xi_{m}$ for which we have

$$
A_{1} \xi_{k}=-c_{k+1} \xi_{k+1}, A_{2} \xi_{k}=\lambda_{k} \xi_{k}, A_{3} \xi_{k}=q c_{k} \xi_{k-1} .
$$

Here, for arbitrary $k$, we set

$$
\lambda_{k}=q^{2}\left(1-q^{2}\right)^{-1}\left(q^{-4 k}-1\right)
$$


and, for $-m \leq k \leq+m$, we set

$$
c_{k}=q\left(1-q^{2}\right)^{-1}\left[\left(q^{-2 k}-q^{2 m}\right)\left(q^{-2 m}-q^{-2(k-1)}\right)\right]^{1 / 2} .
$$

(Of course, $c_{k}$ is dependent on $m$, that is, on $M$, as well as on $k$, but we suppress this dependence in the notation for the sake of simplifying it.)

So that our formulas will always make sense, we define $\xi_{-m-1}=\xi_{m+1}=0$ and $c_{-m-1}=c_{m+1}=0$.

Denote by $W_{p k}^{M}$ the matrix entries of $W^{M}$ relative to the basis $\xi_{-m}, \ldots, \xi_{m}$. To calculate $d\left(W_{p k}^{M}\right)$ we need to calculate $E_{r}\left(W_{p k}^{M}\right)$, for $r=1,2,3$. We have

$$
E_{1}\left(W_{p k}^{M}\right)=\left(\mathrm{id} \otimes \chi_{1}\right) \sum_{i=-m}^{i=+m} W_{p i}^{M} \otimes W_{i k}^{M}=\sum_{i=-m}^{i=+m} A_{1}(i k) W_{p i}^{M}=-c_{k+1} W_{p, k+1}^{M} .
$$

By similar calculations we get the following:

$$
E_{1}\left(W_{p k}^{M}\right)=-c_{k+1} W_{p, k+1}^{M}, E_{2}\left(W_{p k}^{M}\right)=\lambda_{k} W_{p k}^{M}, E_{3}\left(W_{p k}^{M}\right)=q c_{k} W_{p, k-1}^{M} .
$$

So that these formulas always make sense, we define $W_{p,-m-1}^{M}=W_{p, m+1}^{M}=0$. For other purposes below, we also set $W_{p,-m-2}^{M}=W_{p, m+2}^{M}=0$. It follows from the preceding equations that for $k$ in the range $-m, \ldots,+m$,

$$
d W_{p k}^{M}=-c_{k+1} W_{p, k+1}^{M} \eta_{1}+\lambda_{k} W_{p k}^{M} \eta_{2}+q c_{k} W_{p, k-1}^{M} \eta_{3} .
$$

Of course, $T d W_{p k}^{M}=L d^{2} W_{p k}^{M}=0$. Moreover, if $M>1$, then $d W_{p k}^{M}$ is non-zero. Otherwise, $W_{p k}^{M}=c 1$, for some scalar $c$ (this is a "connectedness" result for $S U_{q}(2)$; see [13, Theorem 2.3]). Since $W_{p k}^{M}$ and the unit 1 are orthogonal in $\mathcal{A}_{q}$, because they are matrix elements of inequivalent representations of $S U_{q}(2)$, we must have $c=0$. But then $W_{p k}^{M}=0$, an impossibility, since $W_{p k}^{M}$ belongs to a linear basis of $\mathcal{A}_{q}$. Hence, $d W_{p k}^{M} \neq 0$, as claimed. It follows that $d W_{p k}^{M}$ is an eigenvector for $T$.

From these observations, it is plausible to conjecture that it may be useful to look at the linear space spanned by the vectors occurring in the right-hand side of Equation (11). Indeed, this turns out to be the key to solving the eigenvalue problem for $T$.

For $p=-m,-m+1, \ldots,+m, k=-m-1,-m, \ldots, m+1$, denote by $\mathcal{E}_{1}(M, p, k)$ the linear span of the vectors $W_{p, k+1}^{M} \eta_{1}, W_{p k}^{M} \eta_{2}$ and $W_{p, k-1}^{M} \eta_{3}$. Since $\eta_{1}, \eta_{2}, \eta_{3}$ are orthogonal, so are these spanning vectors. It follows that we have $\operatorname{dim}\left(\mathcal{E}_{1}(M, p, k)\right)=$ 3 , if $k$ is in the range $-m+1, \ldots, m-1$. If $M \neq 1$ and $k=-m$ or $k=+m$, $\operatorname{dim}\left(\mathcal{E}_{1}(M, p, k)\right)=2$. If $M \neq 1$ and $k=-m-1$ or $k=m+1, \operatorname{dim}\left(\mathcal{E}_{1}(M, p, k)\right)=1$. Finally, the spaces $\mathcal{E}_{1}(1,0,-1), \mathcal{E}_{1}(1,0,0)$ and $\mathcal{E}_{1}(1,0,+1)$ all have dimension one also.

Set $f_{1}=W_{p, k+1}^{M} \eta_{1}, f_{2}=W_{p k}^{M} \eta_{2}$ and $f_{3}=W_{p, k-1}^{M} \eta_{3}$.

We shall need the following result.

Lemma 8.2. Let $\omega$ be an element of $\mathcal{E}_{1}(M, p, k)$ that is orthogonal to $d W_{p k}^{M}$. Then $d^{*} \omega=0$.

Proof. We have $\omega=\sum_{r=1}^{3} \alpha_{r} f_{r}$, for some scalars $\alpha_{1}, \alpha_{2}, \alpha_{3}$. So, $d^{*} \omega=\sum_{r=1}^{3} \alpha_{r} d^{*} f_{r}$. Using Equation Set (10), and the relations $E_{1}^{*}=-q^{-1} E_{3}, E_{2}^{*}=E_{2}$ and $E_{3}^{*}=-q E_{1}$, which follow from Lemma 2.3 and Equation Set (8), we calculate that $d^{*} f_{1}=$ $-c_{k+1} W_{p k}^{M}, d^{*} f_{2}=\lambda_{k} W_{p k}^{M}$ and $d^{*} f_{3}=q c_{k} W_{p k}^{M}$. Hence, $d^{*} \omega=\left(-\alpha_{1} c_{k+1}+\alpha_{2} \lambda_{k}\right.$ $\left.+\alpha_{3} q c_{k}\right) W_{p k}^{M}$. However, by hypothesis $\left(d^{*} \omega \mid W_{p k}^{M}\right)=\left(\omega \mid d W_{p k}^{M}\right)=0$. Hence, $d^{*} \omega=0$. 
It is clear that the spaces $\mathcal{E}_{1}(M, p, k)$ are pairwise orthogonal; more precisely, $\mathcal{E}_{1}(M, p, k) \perp \mathcal{E}\left(M^{\prime}, p^{\prime}, k^{\prime}\right)$ if $(M, p, k) \neq\left(M^{\prime}, p^{\prime}, k^{\prime}\right)$, since the elements $W_{p k}^{M}$ are orthogonal in $\mathcal{A}_{q}$ and $\eta_{1}, \eta_{2}, \eta_{3}$ are orthogonal in $\Omega_{1}^{\text {inv }}$. Also, since $\Omega_{1}$ is linearly spanned by the vectors $W_{p k}^{M} \eta_{r}$, it is the orthogonal sum of the spaces $\mathcal{E}_{1}(M, p, k)$; that is,

$$
\Omega_{1}=\bigoplus_{(M, p, k)} \mathcal{E}_{1}(M, p, k),
$$

where $M$ ranges over the positive integers and $p=-m,-m+1, \ldots,+m$ and $k=$ $-m-1,-m, \ldots, m+1$ (and $M=2 m+1$ ). We shall show that all the spaces occurring in this decomposition of $\Omega_{1}$ are invariant under $T$. Hence, we shall have reduced the eigenvalue problem for $T$ to the problem of explicitly calculating $T$ on these spaces of dimension 1,2 or 3 .

The relevance of this to the problem of finding the eigenvalues of $\nabla$ can now be stated very explicitly. We can split $\mathcal{E}_{1}(M, p, k)$ into the sum of $\mathbf{C} d W_{p k}^{M}$ and its orthogonal complement $\mathcal{F}(M, p, k)$. By the same reasoning as we used in Section 5 , it follows from Equation Set (10) that $W_{p k}^{M}$ and $d W_{p k}^{M}$ are eigenvectors of $\nabla$, with eigenvalue $\nu_{k}$ given by

$$
\nu_{k}=c_{k+1}^{2}+\lambda_{k}^{2}+q^{2} c_{k}^{2} .
$$

(For $d W_{p k}^{M}$ to be an eigenvector, we need $M>1$.) Observe that $\nu_{k}>0$, unless $m=k=0$, as is trivially verified. For $\omega \in \mathcal{F}(M, p, k)$, we have $d^{*} \omega=0$, by Lemma 8.2. Hence, $\nabla \omega=d^{*} d \omega=T^{2} \omega$. Therefore, using the invariance of the space $\mathcal{E}_{1}(M, p, k)$ under $T$ that we shall show below, and that $T=T^{*}$, it follows from the equation $T d W_{p k}^{M}=0$ that $\mathcal{F}(M, p, k)$ is invariant under $T$ also and therefore that $\mathcal{F}(M, p, k)$ is invariant under $\nabla$. Moreover, the eigenvalues of $\nabla$ on this space are the squares of the eigenvalues of $T$ on it. Thus, we have reduced the eigenvalue problem for $\nabla$ to the problem of calculating the eigenvalues of $T$ on the spaces $\mathcal{F}(M, p, k)$.

We now calculate the values of $T$ on the vectors $W_{p k}^{M} \eta_{1}, W_{p k}^{M} \eta_{2}$ and $W_{p k}^{M} \eta_{3}$, where $k$ is in the range $-m, \ldots,+m$. We have

$$
\begin{aligned}
T\left(W_{p k}^{M} \eta_{1}\right) & =\sum_{r=1}^{3} T_{r 1}\left(W_{p k}^{M}\right) \eta_{r} \\
& =q^{9}\left(1+q^{2}\right) W_{p k}^{M} \eta_{1}-q^{11} E_{2}\left(W_{p k}^{M}\right) \eta_{1}-q^{6} E_{3}\left(W_{p k}^{M}\right) \eta_{2} \\
& =\left(q^{9}\left(1+q^{2}\right)-q^{11} \lambda_{k}\right) W_{p k}^{M} \eta_{1}-q^{7} c_{k} W_{p, k-1}^{M} \eta_{2},
\end{aligned}
$$

by Equation Set (10). Similarly, one can show that

$$
T\left(W_{p k}^{M} \eta_{2}\right)=-q^{7} c_{k+1} W_{p, k+1}^{M} \eta_{1}+q^{5} W_{p k}^{M} \eta_{2}-q^{4} c_{k} W_{p, k-1}^{M} \eta_{3}
$$

and

$$
T\left(W_{p k}^{M} \eta_{3}\right)=-q^{4} c_{k+1} W_{p, k+1}^{M} \eta_{2}+q\left(1+q^{2}\right) W_{p k}^{M} \eta_{3}+q^{-1} \lambda_{k} W_{p k}^{M} \eta_{3} .
$$

However, it is elementary to show that $q^{9}\left(1+q^{2}\right)-q^{11} \lambda_{k}=-q^{7} \lambda_{k-1}$ and therefore also $q\left(1+q^{2}\right)+q^{-1} \lambda_{k-1}=q^{3} \lambda_{k}$. Hence,

$$
\begin{gathered}
T\left(f_{1}\right)=-q^{7} \lambda_{k} f_{1}-q^{7} c_{k+1} f_{2}, \\
T\left(f_{2}\right)=-q^{7} c_{k+1} f_{1}+q^{5} f_{2}-q^{4} c_{k} f_{3}, \\
T\left(f_{3}\right)=-q^{4} c_{k} f_{2}+q^{3} \lambda_{k} f_{3} .
\end{gathered}
$$

Hence, $\mathcal{E}_{1}(M, p, k)$ is invariant for $T$. 
Now consider the case that $k$ is in the range $-m+1, \ldots, m-1$, so that $f_{1}, f_{2}, f_{3}$ form an orthogonal basis for $\mathcal{E}=\mathcal{E}_{1}(M, p, k)$. Our preceding calculations show that the matrix form of the restriction $T_{\mathcal{E}}$ of $T$ relative to this basis is given by

$$
T_{\mathcal{E}}=\left[\begin{array}{ccc}
-q^{7} \lambda_{k} & -q^{7} c_{k+1} & 0 \\
-q^{7} c_{k+1} & q^{5} & -q^{4} c_{k} \\
0 & -q^{4} c_{k} & q^{3} \lambda_{k}
\end{array}\right] .
$$

By Equation (11), $d W_{p k}^{M}$ belongs to $\mathcal{E}$ and we showed above that $T d W_{p k}^{M}=0$. It therefore remains only to calculate the eigenvalues of $T_{\mathcal{E}}$ on $\mathcal{F}_{1}=\mathcal{F}(M, p, k)$.

Let us write

$$
\left[\begin{array}{ccc}
x & s & 0 \\
s & y & t \\
0 & t & z
\end{array}\right]=T_{\mathcal{E}}=\left[\begin{array}{ccc}
-q^{7} \lambda_{k} & -q^{7} c_{k+1} & 0 \\
-q^{7} c_{k+1} & q^{5} & -q^{4} c_{k} \\
0 & -q^{4} c_{k} & q^{3} \lambda_{k}
\end{array}\right] .
$$

Then the characteristic polynomial $P(\lambda)=\operatorname{det}\left(T_{\mathcal{E}}-\lambda 1\right)$ of $T_{\mathcal{E}}$ is given by

$$
P(\lambda)=-\lambda^{3}+(x+y+z) \lambda^{2}-\left(x y+y z+x z-s^{2}-t^{2}\right) \lambda+x y z-t^{2} x-s^{2} z .
$$

Since 0 is an eigenvalue of $T_{\mathcal{E}}$, we must have $x y z-t^{2} x-s^{2} z=0$. Hence,

$$
P(\lambda)=-\lambda\left(\lambda^{2}-(x+y+z) \lambda+x y+y z+x z-s^{2}-t^{2}\right) .
$$

Set $B_{k}=x+y+z$ and $C_{k}=x y+y z+x z-s^{2}-t^{2}$. Then the eigenvalues of $T$ on $\mathcal{F}_{1}$ are given by

$$
\mu_{k}^{ \pm}=\left(B_{k} \pm \sqrt{B_{k}^{2}-4 C_{k}}\right) / 2 .
$$

Explicitly,

$$
B_{k}=\left(q^{3}-q^{7}\right) \lambda_{k}+q^{5}
$$

and

$$
C_{k}=q^{8}\left[\left(1-q^{4}\right) \lambda_{k}-q^{2} \lambda_{k}^{2}-q^{6} c_{k+1}^{2}-c_{k}^{2}\right]=-q^{10} \nu_{k} .
$$

The second equality in Equation (15) follows from Equation (12) and the first equality in (15) together with the easily-checked fact that $\lambda_{k}=c_{k}^{2}-q^{2} c_{k+1}^{2}$.

As observed above, the eigenvalues of $\nabla$ on $\mathcal{F}(M, p, k)$ are the squares of those of $T$, that is, $\left(\mu_{k}^{+}\right)^{2}$ and $\left(\mu_{k}^{-}\right)^{2}$.

We turn now to the case where $\mathcal{E}=\mathcal{E}_{1}(M, p, k)$ is 2-dimensional; that is, where $M>1$ and $k=-m$ or $k=+m$.

Suppose first that $k=-m$. Then $f_{3}=0$ and $f_{1}, f_{2}$ form an orthogonal basis of $\mathcal{E}$. Hence,

$$
T_{\mathcal{E}}=\left[\begin{array}{cc}
-q^{7} \lambda_{k} & -q^{7} c_{k+1} \\
-q^{7} c_{k+1} & q^{5}
\end{array}\right] .
$$

Since one eigenvalue of this matrix is zero (because $T d W_{p k}^{M}=0$ ), the other eigenvalue on $\mathcal{F}_{1}(M, p,-m)$ is the sum of the diagonal entries $q^{5}-q^{7} \lambda_{k}$.

Similar considerations show that $T$ has eigenvalue $q^{5}+q^{3} \lambda_{k}$ on $\mathcal{F}_{1}(M, p,+m)$.

Finally, we consider the trivial case where $\mathcal{E}_{1}(M, p, k)$ is 1-dimensional: If $M>1$, then $k=-m-1$ or $k=m+1$, and it is trivially checked that the eigenvalue of $T$ on $\mathcal{E}_{1}(M, p, k)$ is $-q^{7} \lambda_{k}$ or $q^{3} \lambda_{k}$, respectively. If $M=1$, then $\mathcal{E}_{1}(M, p, k)=$ $\mathcal{E}_{1}(1,0, k)=\mathcal{F}_{1}(1,0, k)$. Clearly, $\mathcal{F}_{1}(1,0,-1)=\mathbf{C} \eta_{1}$ and we computed earlier that $T \eta_{1}=q^{9}\left(1+q^{2}\right) \eta_{1}$; similarly, $\mathcal{F}_{1}(1,0,0)=\mathbf{C} \eta_{2}$ and $T\left(\eta_{2}\right)=q^{5} \eta_{2}$; finally, we have $\mathcal{F}_{1}(1,0,+1)=\mathbf{C} \eta_{3}$ and $T \eta_{3}=q\left(1+q^{2}\right) \eta_{3}$. 
This completes the computation of the eigenvalues of $T$ and therefore of the eigenvalues of $\nabla$ on $\Omega_{1}$. We summarize our results:

The space $\Omega_{1}$ is the direct sum of the spaces $\mathcal{E}_{1}(M, p, k)$; that is,

$$
\Omega_{1}=\bigoplus_{(M, p, k)} \mathcal{E}_{1}(M, p, k)
$$

and the spaces $\mathcal{E}_{1}(M, p, k)$ are invariant under $\nabla$. Hence, the eigenvalue problem for $\nabla$ is reduced to the problem of calculating the eigenvalues on these spaces of dimension 1, 2 or 3 .

Case 1: If $\operatorname{dim} \mathcal{E}_{1}(M, p, k)=1$ and $M>1$, then $\nabla$ has eigenvalue $q^{14} \lambda_{k}^{2}$ or $q^{6} \lambda_{k}^{2}$ on $\mathcal{E}_{1}(M, p, k)$; if $\operatorname{dim} \mathcal{E}_{1}(M, p, k)=1$ and $M=1$, then $\nabla$ has eigenvalues $q^{18}\left(1+q^{2}\right)^{2}, q^{10}$ and $q^{2}\left(1+q^{2}\right)^{2}$ on $\mathcal{E}_{1}(1,0,-1), \mathcal{E}_{1}(1,0,0)$ and $\mathcal{E}_{1}(1,0,+1)$, respectively.

Case 2: If $\operatorname{dim} \mathcal{E}_{1}(M, p, k)=2$, then, on $\mathcal{E}_{1}(M, p, k), \nabla$ has eigenvalue $\nu_{k}$ and either $\left(q^{5}-q^{7} \lambda_{k}\right)^{2}$, if $k=-m$, or $\left(q^{5}+q^{3} \lambda_{k}\right)^{2}$, if $k=+m$.

Case 3: If $\operatorname{dim} \mathcal{E}_{1}(M, p, k)=3$, then $\nabla$ has eigenvalues $\nu_{k},\left(\mu_{k}^{+}\right)^{2}$ and $\left(\mu_{k}^{-}\right)^{2}$, where

$$
\mu_{k}^{ \pm}=\left(B_{k} \pm \sqrt{B_{k}^{2}-4 C_{k}}\right) / 2
$$

with

$$
B_{k}=\left(q^{3}-q^{7}\right) \lambda_{k}+q^{5} \quad \text { and } \quad C_{k}=-q^{10} \nu_{k}
$$

Because of our choice of inner product, the Hodge operator $L$ commutes with the Laplacian $\nabla$. Hence, the eigenvalues of $\nabla$ on $\Omega_{2}$ are the same as those on $\Omega_{1}$. The eigenvalues of $\nabla$ on $\Omega_{0}$ are determined by the equation $\nabla W_{p k}^{M}=\nu_{k} W_{p k}^{M}$. Since $L\left(\Omega_{0}\right)=\Omega_{3}$, and $L$ commutes with $\nabla$, the eigenvalues of $\nabla$ on $\Omega_{3}$ are the same as those on $\Omega_{0}$. We have therefore calculated all the eigenvalues of $\nabla$.

Now we turn to the question of trying to fit this three-dimensional differential calculus into the framework of Connes' non-commutative differential geometry. For this we shall need to obtain some asymptotic estimates for the eigenvalues we have just computed.

Theorem 8.3. There exists a positive constant $C(q)$, depending only on $q$ and not on $M, p$ and $k$, such that $\nabla \geq C(q) \max \left(q^{-8 k}, 1\right)$ on $\mathcal{E}_{1}(M, p, k)$.

Proof. Since $\mathcal{E}_{1}(M, p, k)$ admits an orthonormal basis of eigenvectors of $\nabla$, we need only show that if $\mu$ is an eigenvalue of $\nabla$ on $\mathcal{E}_{1}(M, p, k)$, then $\mu \geq C(q) \max \left(q^{-8 k}, 1\right)$. We shall prove this only in the case that $\operatorname{dim}\left(\mathcal{E}_{1}(M, p, k)\right)=3$. The cases where $\operatorname{dim}\left(\mathcal{E}_{1}(M, p, k)\right)$ is equal to 2 or 1 can be dealt with similarly, but much more simply.

We first need to establish some lower bounds for $\lambda_{k}^{2}$, when $k \neq 0$. If $k>0$, then $k \geq 1 / 2$ and therefore $q^{-4 k} \geq q^{-2}$, from which it follows immediately that $\lambda_{k} \geq 1$. If $k<0$, then $k \leq-1 / 2$ and therefore $q^{-4 k} \leq q^{2}$. Hence, $\lambda_{k} \leq-q^{2}$. In either case, 
$\lambda_{k}^{2} \geq q^{4}$, provided $k \neq 0$. Moreover,

$$
\begin{aligned}
\frac{q^{-8 k}}{\lambda_{k}^{2}} & =\frac{q^{-8 k}-2 q^{-4 k}+1}{\lambda_{k}^{2}}+2 \frac{q^{-4 k}-1}{\lambda_{k}^{2}}+\frac{1}{\lambda_{k}^{2}} \\
& =\frac{\left(1-q^{2}\right)^{2}}{q^{4}} \frac{\lambda_{k}^{2}}{\lambda_{k}^{2}}+\frac{2\left(1-q^{2}\right)}{q^{2}} \frac{\lambda_{k}}{\lambda_{k}^{2}}+\frac{1}{\lambda_{k}^{2}} \\
& \leq \frac{\left(1-q^{2}\right)^{2}}{q^{4}}+\frac{2\left(1-q^{2}\right)}{q^{2}} \frac{1}{\left|\lambda_{k}\right|}+\frac{1}{\lambda_{k}^{2}} \\
& \leq \frac{\left(1-q^{2}\right)^{2}}{q^{4}}+\frac{2\left(1-q^{2}\right)}{q^{4}}+\frac{1}{q^{4}}=\frac{\left(2-q^{2}\right)^{2}}{q^{4}} .
\end{aligned}
$$

Since $q^{4} \geq q^{4}\left(2-q^{2}\right)^{-2}$, we have $\lambda_{k}^{2} \geq q^{4}\left(2-q^{2}\right)^{-2} \max \left(q^{-8 k}, 1\right)$, provided $k \neq 0$. Since $\nu_{k} \geq \lambda_{k}^{2}$, by Equation (12), this gives lower bounds for $\nu_{k}$ also, when $k \neq 0$. If $k=0$, we use the inequality $\nu_{0} \geq q^{2} c_{0}^{2}$. Since $\operatorname{dim} \mathcal{E}_{1}(M, p, k)=3$, we have $m \geq 1$ and therefore $q^{2 m} \leq q^{2}$ and $q^{-2 m} \geq q^{-2}$. Hence, we have $c_{0}^{2} \geq$ $q^{2}\left(1-q^{2 m}\right)\left(q^{-2}-q^{2}\right)\left(1-q^{2}\right)^{-2}=\left(1-q^{2 m}\right)\left(1+q^{2}\right)\left(1-q^{2}\right)^{-1} \geq 1+q^{2}$. Thus, $\nu_{0} \geq q^{4}$. Therefore, for all $k$,

$$
\nu_{k} \geq a(q) \max \left(q^{-8 k}, 1\right), \quad \text { where } \quad a(q)=q^{4}\left(2-q^{2}\right)^{-2} .
$$

We turn now to the question of obtaining similar inequalities for the other two eigenvalues of $\nabla$ on $\mathcal{E}_{1}(M, p, k)$. Recall that these are $\left(\mu_{k}^{+}\right)^{2}$ and $\left(\mu_{k}^{-}\right)^{2}$, where $\mu_{k}^{ \pm}$ are given by Equation (13). Hence, if $\mu$ is one of $\mu^{+}$or $\mu^{-}$, then

$$
\mu^{2}=\left(X_{k} \pm \sqrt{X_{k}^{2}-4 C_{k}^{2}}\right) / 2,
$$

where we set $X_{k}=B_{k}^{2}-2 C_{k}$. It follows from Equations (14) and (15) that

$$
X_{k}=q^{6}\left[\left(1+q^{8}\right) \lambda_{k}^{2}+q^{4}+2 q^{8} c_{k+1}^{2}+2 q^{2} c_{k}^{2}\right]
$$

In particular, $X_{k} \neq 0$, since $X_{k} \geq q^{10}$. Note that $\mu^{2} \geq\left(X_{k}-\sqrt{X_{k}^{2}-4 C_{k}^{2}}\right) / 2=$ $2 C_{k}^{2}\left(X_{k}+\sqrt{X_{k}^{2}-4 C_{k}^{2}}\right)^{-1} \geq C_{k}^{2} / X_{k}$, because $\sqrt{X_{k}^{2}-4 C_{k}^{2}} \leq X_{k}$. Since $C_{k}=$ $-q^{10} \nu_{k}$ and $\nu_{k} \neq 0$, therefore $C_{k} \neq 0$. Also, since $C_{k}^{2}=q^{20} \nu_{k}^{2}$, it follows that $C_{k}^{2}$ is greater than or equal to each of the numbers $q^{20} \lambda_{k}^{4}, q^{20} \lambda_{k}^{2} c_{k+1}^{2}$ and $q^{22} \lambda_{k}^{2} c_{k}^{2}$, by Equation (12). Moreover, $C_{k}^{2} \geq q^{20} a(q) \nu_{k} \geq q^{20} a(q)^{2} q^{-8 k}$. Using these inequalities and the inequality $q^{-8 k} / \lambda_{k}^{2} \leq 1 / a(q)$, where $k \neq 0$, that we established above, we get

$$
\begin{aligned}
\frac{q^{-8 k}}{\mu^{2}} & \leq \frac{X_{k} q^{-8 k}}{C_{k}^{2}}=\frac{q^{-8 k} q^{6}\left[\left(1+q^{8}\right) \lambda_{k}^{2}+q^{4}+2 q^{8} c_{k+1}^{2}+2 q^{2} c_{k}^{2}\right]}{C_{k}^{2}} \\
& \leq q^{6}\left[\left(1+q^{8}\right) \frac{q^{-8 k}}{q^{20} \lambda_{k}^{2}}+q^{4} \frac{q^{-8 k}}{C_{k}^{2}}+2 q^{8} \frac{q^{-8 k}}{q^{20} \lambda_{k}^{2}}+2 q^{2} \frac{q^{-8 k}}{q^{22} \lambda_{k}^{2}}\right] \\
& \leq q^{6}\left[\left(1+q^{8}\right) q^{-20} a(q)^{-1}+q^{-16} a(q)^{-2}+2 q^{-12} a(q)^{-1}+2 q^{-20} a(q)^{-1}\right] .
\end{aligned}
$$

If we denote the reciprocal of the right-hand side of this last inequality by $b_{1}(q)$, then we have shown that $\mu^{2} \geq b_{1}(q) q^{-8 k}$, whenever $k \neq 0$. 
If we leave out the factor $q^{-8 k}$ in the above inequalities and use the fact that, for $k \neq 0$, we have $\lambda_{k}^{2} \geq q^{4}$ and therefore $C_{k}^{2} \geq q^{28}$, we get

$$
\begin{aligned}
\frac{1}{\mu^{2}} & \leq q^{6}\left[\left(1+q^{8}\right) \frac{1}{q^{20} \lambda_{k}^{2}}+\frac{q^{4}}{C_{k}^{2}}+2 q^{8} \frac{1}{q^{20} \lambda_{k}^{2}}+2 q^{2} \frac{1}{q^{22} \lambda_{k}^{2}}\right] \\
& \leq q^{6}\left[\left(1+q^{8}\right) \frac{1}{q^{20} q^{4}}+q^{4} \frac{1}{q^{28}}+2 q^{8} \frac{1}{q^{20} q^{4}}+2 q^{2} \frac{1}{q^{22} q^{4}}\right]
\end{aligned}
$$

Hence, if $b_{2}(q)$ denotes the reciprocal of the right-hand side of the last inequality, we have shown that $\mu^{2} \geq b_{2}(q)$.

Finally, we have to consider the case where $k=0$. (Then $\lambda_{k}=0$ and we cannot use the preceding estimates.) However, in this case, we have $C_{0}^{2}=q^{20} \nu_{0}^{2}=$ $q^{20}\left(c_{1}^{2}+q^{2} c_{0}^{2}\right)^{2}$, by Equation (12); hence, $C_{0}^{2} \geq q^{20} c_{1}^{4} \geq q^{20} c_{1}^{2}$ and $C_{0}^{2} \geq q^{24} c_{0}^{4} \geq$ $q^{26} c_{0}^{2}$. Also, since $\nu_{0} \geq a(q)$, we have $C_{0}^{2} \geq q^{20} a(q)^{2}$. It follows from these inequalities that

$$
\frac{1}{\mu^{2}} \leq \frac{X_{0}}{C_{0}^{2}}=q^{6}\left[\frac{q^{4}}{C_{0}^{2}}+\frac{2 q^{8} c_{1}^{2}}{C_{0}^{2}}+\frac{2 q^{2} c_{0}^{2}}{C_{0}^{2}}\right] \leq q^{6}\left[\frac{q^{4}}{q^{20} a(q)^{2}}+\frac{2 q^{8}}{q^{20}}+\frac{2 q^{2}}{q^{26}}\right] .
$$

Thus, $\mu^{2} \geq b_{3}(q)$, where $b_{3}(q)$ is the reciprocal of the right-hand side of the last inequality.

Set $b(q)=\min \left(b_{1}(q), b_{2}(q), b_{3}(q)\right)$. Then $b(q)$ is a positive constant depending only on $q$ and not on $M, p$ and $k$ and, for $\mu=\mu_{k}^{ \pm}$, we have shown that $\mu^{2} \geq$ $b(q) \max \left(q^{-8 k}, 1\right)$, for all $k$. To complete the proof (in the case that $\operatorname{dim}\left(\mathcal{E}_{1}(M, p, k)\right)$ $=3)$, take $C(q)=\min (a(q), b(q))$.

We now define a decomposition for the space $\Omega$ that is analogous to that given for $\Omega_{1}$ : If $m$ is a non-negative integer or half-integer, $M=2 m+1$ and if $p, k=$ $-m,-m+1, \ldots,+m$, set $\mathcal{E}_{0}(M, p, k)=\mathbf{C} W_{p k}^{M}$; if $k=-m-1$ or $k=m+1$, set $\mathcal{E}_{0}(M, p, k)=0$. Set $\mathcal{E}_{2}(M, p, k)=L\left(\mathcal{E}_{1}(M, p, k)\right)$ and $\mathcal{E}_{3}(M, p, k)=L\left(\mathcal{E}_{0}(M, p, k)\right)$. We have

$$
\Omega=\bigoplus_{(M, p, k)} \mathcal{E}(M, p, k)
$$

where $\mathcal{E}(M, p, k)$ denotes the orthogonal sum $\bigoplus_{i=0}^{3} \mathcal{E}_{i}(M, p, k)$. It follows from Theorem 8.3 that there exists a positive constant $C(q)$, depending only on $q$ and not on $M, p$ or $k$, such that, for $M>1$, the restriction $\nabla_{\mathcal{E}}$ of $\nabla$ to $\mathcal{E}(M, p, k)$ satisfies the following inequalities:

$$
\nabla_{\mathcal{E}} \geq C(q), \quad \nabla_{\mathcal{E}} \geq C(q) q^{-8 k}, \quad \nabla_{\mathcal{E}} \geq C(q) q^{-4 k}
$$

For $M=1$, these inequalities hold for $\nabla_{\mathcal{E}}$ equal to the restriction of $\nabla$ to $\mathcal{E}$, where $\mathcal{E}=\mathcal{E}_{1}(1, p, k) \oplus \mathcal{E}_{2}(1, p, k)\left(\nabla=0\right.$ on $\left.\mathcal{E}_{0}(1, p, k) \oplus \mathcal{E}_{3}(1, p, k)\right)$. The third of the inequalities in (17) is an easy consequence of the first two. The required inequalities for $\mathcal{E}_{0}(M, p, k)$, when this space is non-zero, and $M>1$, follow from the fact that the eigenvalue of $\nabla$ on $\mathcal{E}_{0}(M, p, k)=\mathbf{C} W_{p k}^{M}$ is $\nu_{k}$, which is also an eigenvalue of $\nabla$ on the corresponding space $\mathcal{E}_{1}(M, p, k)$. We are also using the fact that the eigenvalues of $\nabla$ on $\mathcal{E}_{2}(M, p, k)$ and $\mathcal{E}_{3}(M, p, k)$ are the same as those on $\mathcal{E}_{1}(M, p, k)$ and $\mathcal{E}_{0}(M, p, k)$, respectively.

We set

$$
\mathcal{G}(M, k)=\bigoplus_{p=-m}^{p=+m} \mathcal{E}(M, p, k) \quad \text { for } \quad k=-m-1,-m, \ldots, m, m+1
$$


Then $\Omega=\bigoplus_{(M, k)} \mathcal{G}(M, k)$, as an orthogonal sum by the orthogonality properties of the $W^{M}$.

Since $\mathcal{G}(M, k)$ is finite dimensional and $\nabla$ is self-adjoint, the restriction $\nabla_{(M, k)}$ to $\mathcal{G}(M, k)$ is diagonalisable. The inequalities in (17), for $M>1$, imply corresponding inequalities for the eigenvalues of $\nabla_{(M, k)}$, from which it follows that

$$
\nabla_{(M, k)} \geq C(q), \quad \nabla_{(M, k)} \geq C(q) q^{-8 k}, \quad \nabla_{(M, k)} \geq C(q) q^{-4 k} .
$$

Now define a bounded operator $R$ on $\Omega$ by setting $R=(1+\nabla)^{-1 / 2}$. This is to be understood more precisely as saying that $R$ is the direct sum of the operators $R_{(M, k)}$, where $R_{(M, k)}=\left(1+\nabla_{(M, k)}\right)^{-1 / 2}$. Since $R_{(M, k)} \leq 1$, we have $\|R\| \leq 1$. However, we can do better: it follows easily from the inequalities in (18) that

$$
\left\|R_{(M, k)}\right\| \leq C(q)^{-1 / 2} \min \left(q^{2 k}, q^{4 k}\right) .
$$

(We can easily modify $C(q)$ by making it smaller so that (19) also holds for $M=1$.)

We recall from [13, Equation 2.16] that there are linear functionals $f_{1}, f_{2}, f_{3}$ on $\mathcal{A}_{q}$ such that $\eta_{r} a=\left(\operatorname{id} \otimes f_{r}\right) \Delta(a) \eta_{r}$, for all $a \in \mathcal{A}_{q}$. Moreover, $f_{3}=f_{1}$ and $\left(f_{1} \otimes f_{1}\right) \Delta=f_{2}=\varepsilon+\left(q^{-2}-1\right) \chi_{2}$, where $\varepsilon$ is the co-unit of $\mathcal{A}_{q}$.

Set $B_{r}=f_{r}\left(W^{M}\right)$. Then $\left(B_{2}\right)_{p k}=\delta_{p k}+\left(q^{-2}-1\right) \lambda_{k} \delta_{p k}=q^{-4 k} \delta_{p k}$. Also, $B_{1}=B_{3}$ and $B_{1}^{2}=B_{2}$. Since $B_{2}$ is diagonal, with distinct diagonal entries, and $B_{1}$ commutes with $B_{2}$, therefore $B_{1}$ is also diagonal. In fact, the diagonal entries of $B_{1}$ are positive [13, Equation 5.53], so $\left(B_{1}\right)_{p k}=q^{-2 k} \delta_{p k}$. Hence, for all $r$,

$$
\eta_{r} W_{p k}^{M}=\left(\mathrm{id} \otimes f_{r}\right) \Delta\left(W_{p k}^{M}\right) \eta_{r}=\left(W^{M} B_{r}\right)_{p k} \eta_{r}=q^{-2 \varepsilon_{r} k} W_{p k}^{M} \eta_{r},
$$

where $\varepsilon_{1}=\varepsilon_{3}=1$ and $\varepsilon_{2}=2$.

Suppose that $M$ is an integer, $M=2 m+1$, and that $k$ is a half-integer or integer. We define $\mathcal{G}(M, k)=0$, if $M \leq 0$ or if $k$ does not belong to the set $\{-m-1,-m, \ldots, m, m+1\}$.

Lemma 8.4. There exists a positive number $C(q)$ (depending on $q$ but not on $M$ or $k)$ such that, for $M \geq 1$ and $k=-m-1,-m, \ldots, m, m+1$, we have:

1. $M_{1}(\mathcal{G}(M, k)) \subseteq \mathcal{G}(M, k-1)$ and $\left\|\left(M_{1}\right)_{\mathcal{G}(M, k)}\right\| \leq C(q) q^{-2 k}$;

2. $M_{2}(\mathcal{G}(M, k)) \subseteq \mathcal{G}(M, k)$ and $\left\|\left(M_{2}\right)_{\mathcal{G}(M, k)}\right\| \leq C(q) q^{-4 k}$;

3. $M_{3}(\mathcal{G}(M, k)) \subseteq \mathcal{G}(M, k+1)$ and $\left\|\left(M_{3}\right)_{\mathcal{G}(M, k)}\right\| \leq C(q) q^{-2 k}$.

Proof. The subset inclusions $M_{1}(\mathcal{E}(M, p, k)) \subseteq \mathcal{E}(M, p, k-1), M_{2}(\mathcal{E}(M, p, k)) \subseteq$ $\mathcal{E}(M, p, k)$ and $M_{3}(\mathcal{E}(M, p, k)) \subseteq \mathcal{E}(M, p, k+1)$ are easy consequences of Equation Set (20). The subset inclusions in Conditions 1-3 follow immediately. The space $\mathcal{E}(M, p, k)$ is the linear span of the orthogonal vectors

$$
\begin{aligned}
& \begin{array}{cccccc} 
& g_{1}^{p} & \multicolumn{4}{c}{W_{p k}^{M}} \\
g_{2}^{p} & g_{3}^{p} & g_{4}^{p} \\
g_{5}^{p} & g_{6}^{p} & g_{7}^{p} \\
& g_{8}^{p} & & W_{p, k+1}^{M} \eta_{1} & W_{p k}^{M} \eta_{2} & W_{p, k-1}^{M} \eta_{3} \\
& W_{p, k+1}^{M} L\left(\eta_{1}\right) & W_{p k}^{M} L\left(\eta_{2}\right) & W_{p, k-1}^{M} L\left(\eta_{3}\right) \\
& & & W_{p k}^{M} L(1) &
\end{array} \\
& \begin{array}{ccc} 
& W_{p k}^{M} \\
W_{p, k+1}^{M} \eta_{1} & W_{p k}^{M} \eta_{2} & W_{p, k-1}^{M} \eta_{3} \\
q^{-7} W_{p, k+1}^{M} \eta_{1} \eta_{2} & -q^{-6} W_{p k}^{M} \eta_{3} \eta_{1} & q W_{p, k-1}^{M} \eta_{2} \eta_{3} \\
& W_{p k}^{M} \theta . &
\end{array}
\end{aligned}
$$

Hence, setting $b_{k}=\left(B_{1}\right)_{k k}=q^{-2 k}$ and $b_{-m-1}=0$, and using Equation Set (20) and the commutation relations for the products of the elements $\eta_{1}, \eta_{2}$ and $\eta_{3}$, we 
get that the images of the above vectors under $M_{1}$ are given by

$$
\begin{array}{ccc} 
& b_{k} W_{p k}^{M} \eta_{1} & \\
0 & -q^{-4} b_{k} W_{p k}^{M} \eta_{2} \eta_{1} & -q^{-2} b_{k-1} W_{p, k-1}^{M} \eta_{3} \eta_{1} \\
0 & 0 & q^{-5} b_{k-1} W_{p, k-1}^{M} \eta_{2} \eta_{3} \eta_{1} \\
& 0 &
\end{array}
$$

that is, by

$$
\begin{array}{ccc} 
& b_{k} g_{1}^{p} \eta_{1} & \\
0 & -q^{-4} b_{k} g_{3}^{p} \eta_{1} & -q^{-2} b_{k-1} g_{4}^{p} \eta_{1} \\
0 & 0 & q^{-6} b_{k-1} g_{7}^{p} \eta_{1} \\
& 0 &
\end{array}
$$

Now let $Z_{r}$ be the linear span of the vectors $g_{r}^{-m}, g_{r}^{-m+1}, \ldots, g_{r}^{m}$. Note that the linear map, $R_{\eta_{1}}: \omega \mapsto \omega \eta_{1}$, is bounded, since it is the tensor product of the identity map on $\mathcal{A}_{q}$ and its own restriction to $\Omega^{\mathrm{inv}}$, and the latter is bounded since $\Omega^{\text {inv }}$ is finite dimensional. The norm of $R_{\eta_{1}}$ clear depends only on $q$ (provided we fix, as we have, the inner product on $\left.\Omega^{\text {inv }}\right)$. Then, if $\omega \in Z_{1}, M_{1}(\omega)=b_{k} \omega \eta_{1}$, so that $\left\|\left(M_{1}\right)_{Z_{1}}\right\| \leq b_{k}\left\|R_{\eta_{1}}\right\|$. Similarly, $\left\|\left(M_{1}\right)_{Z_{3}}\right\| \leq q^{-4} b_{k}\left\|R_{\eta_{1}}\right\|,\left\|\left(M_{1}\right)_{Z_{4}}\right\| \leq$ $q^{-2} b_{k-1}\left\|R_{\eta_{1}}\right\|,\left\|\left(M_{1}\right)_{Z_{7}}\right\| \leq q^{-6} b_{k-1}\left\|R_{\eta_{1}}\right\|$ and $M_{1}=0$ on $Z_{2}, Z_{5}, Z_{6}$ and $Z_{8}$. Now $\mathcal{G}(M, k)$ is the orthogonal direct sum of the spaces $Z_{r}$, that is, $\mathcal{G}(M, k)=\bigoplus_{r=1}^{8} Z_{r}$. It follows that

$$
\begin{aligned}
\left\|\left(M_{1}\right)_{\mathcal{G}(M, k)}\right\| & =\max _{r}\left(\left\|\left(M_{1}\right)_{Z_{r}}\right\|\right) \leq \max \left(b_{k}, q^{-4} b_{k}, q^{-2} b_{k-1}, q^{-6} b_{k-1}\right)\left\|R_{\eta_{1}}\right\| \\
& \leq \max \left(q^{-2 k}, q^{-4} q^{-2 k}, q^{-2 k}, q^{-4} q^{-2 k}\right)\left\|R_{\eta_{1}}\right\| .
\end{aligned}
$$

Setting $a_{1}(q)=\max \left(1, q^{-4}\right)\left\|R_{\eta_{1}}\right\|$, we get $\left\|\left(M_{1}\right)_{\mathcal{G}(M, k)}\right\| \leq a_{1}(q) q^{-2 k}$.

One can obtain, by similar methods, positive numbers $a_{2}(q)$ and $a_{3}(q)$ such that $\left\|\left(M_{2}\right)_{\mathcal{G}(M, k)}\right\| \leq a_{2}(q) q^{-4 k}$ and $\left\|\left(M_{3}\right)_{\mathcal{G}(M, k)}\right\| \leq a_{3}(q) q^{-2 k}$. Setting $C(q)=$ $\max \left(a_{1}(q), a_{2}(q), a_{3}(q)\right)$, we get the inequalities in Conditions 1-3.

If $a \in \mathcal{A}_{q}$, we denote by $L_{a}$ the bounded operator on $\Omega$ obtained by left multiplication by $a$.

Theorem 8.5. Let $\beta$ and $\delta$ be non-negative numbers for which $\beta+\delta=1$. Then $R^{\beta}\left[L_{a}, D\right] R^{\delta}$ is a bounded operator on $\Omega$, for all $a \in \mathcal{A}_{q}$.

Proof. We shall prove that $R^{\beta}\left[L_{a}, d\right] R^{\delta}$ is bounded, for all $a \in \mathcal{A}_{q}$. This will suffice, since then, replacing $a$ by $a^{*}$ and interchanging the roles of $\beta$ and $\delta$, and using the fact that $L_{a}^{*}=L_{a^{*}}$, we get that the adjoint $-R^{\beta}\left[L_{a}, d^{*}\right] R^{\delta}$ of $R^{\delta}\left[L_{a^{*}}, d\right] R^{\beta}$ is bounded. Hence, $R^{\beta}\left[L_{a}, D\right] R^{\delta}$ is bounded.

Since $\left[L_{a}, d\right](\omega)=-(d a) \omega$, for all $\omega \in \Omega$, we have $\left[L_{a}, d\right]=-\sum_{r=1}^{3} L_{\chi_{r} * a} M_{r}$. Consequently, it suffices to show that $R^{\beta} L_{a} M_{r} R^{\delta}$ is bounded, for all $a \in \mathcal{A}_{q}$ and $r=1,2,3$.

Let $\mathcal{T}$ denote the set of all bounded operators in $B(\Omega)$ for which there exist $N \in \mathbf{Z}$ and $l \in \frac{1}{2} \mathbf{Z}$ such that $T \mathcal{G}(M, k) \subseteq \mathcal{G}(M+N, k+l)$, for all $M \in \mathbf{Z}$ and $k \in \frac{1}{2} \mathbf{Z}$. One easily checks that $\mathcal{T}$ is self-adjoint and closed under multiplication, so that its linear span $\mathcal{T}^{\prime}$ is a self-adjoint subalgebra of $B(\Omega)$. Hence, by the following lemma, $L_{a} \in \mathcal{T}^{\prime}$, for all $a \in \mathcal{A}_{q}$. Thus, to prove the theorem, we need only show now that $R^{\beta} T M_{r} R^{\delta}$ is bounded, for all $T \in \mathcal{T}$. We shall show this only in the case that $r=1$. The cases where $r=2$ or $r=3$ have similar proofs. 
Before proceeding let us first note that by combining Inequality (19) and the inequality in Condition 1 of Lemma 8.4 we get a positive constant $C$ depending only on $q$ such that $\left\|R_{(M, k)}\right\| \leq C q^{2 k}$ and $\left\|\left(M_{1}\right)_{\mathcal{G}(M, k)}\right\| \leq C q^{-2 k}$.

Suppose then $T \in \mathcal{T}$ and $N \in \mathbf{Z}$ and $l \in \frac{1}{2} \mathbf{Z}$ are such that for all $M=1,2, \ldots$ and $k=-m-1, m, \ldots, m, m+1$, we have $T \mathcal{G}(M, k) \subseteq \mathcal{G}(M+N, k+l)$. Let $\omega \in \mathcal{G}(M, k)$. Then $T M_{1} R^{\delta} \omega \in \mathcal{G}(M+N, k+l-1)$. Hence,

$$
\begin{aligned}
\left\|R^{\beta} T M_{1} R^{\delta} \omega\right\|^{2} & \leq C^{2 \beta} q^{4(k+l-1) \beta}\left\|T M_{1} R^{\delta} \omega\right\|^{2} \\
& \leq C^{2 \beta} q^{4(k+l-1) \beta}\|T\|^{2} C^{2} q^{-4 k}\left\|R^{\delta} \omega\right\|^{2} \\
& \leq C^{2 \beta} q^{4(k+l-1) \beta}\|T\|^{2} C^{2} q^{-4 k} C^{2 \delta} q^{4 k \delta}\|\omega\|^{2} \\
& =C^{2(\beta+\delta)} C^{2}\|T\|^{2} q^{4 k(\beta+\delta)} q^{4(l-1) \beta} q^{-4 k}\|\omega\|^{2} \\
& =C^{4}\|T\|^{2} q^{4(l-1) \beta}\|\omega\|^{2} .
\end{aligned}
$$

It follows now, from orthogonality of the image spaces $R^{\beta} T M_{1} R^{\delta} \mathcal{G}(M, k)$, that $R^{\beta} T M_{1} R^{\delta}$ is bounded.

Lemma 8.6. If $\alpha$ and $\gamma$ are the canonical generators of $\mathcal{A}_{q}$ (see [13]), then $L_{\alpha}$ and $L_{\gamma}$ belong to the linear span of the set $\mathcal{T}$ defined in the preceding proof.

Let us introduce some extra notation. We will use the elements $x_{k}$ introduced in the proof of [13, Theorem 5.8], but add an extra parameter into the notation. Let $m \in \frac{1}{2} \mathbf{Z}$. Then we define $x_{k}^{m}=\alpha^{m+k}\left(\gamma^{*}\right)^{m-k}$ for $k=-m, \ldots, m$.

For $k \in \mathbf{Z}$ and $y \in \mathcal{A}_{q}$ we set $y(k)=y^{k}$ if $k \geq 0$ and $y(k)=\left(y^{*}\right)^{-k}$ if $k<0$. We also define $a(k, l)=\alpha(k) \gamma(l)$ for all $k, l \in \mathbf{Z}$. It follows from [13, Theorem 1.2] that the family $\left\{a(k, l)\left(\gamma^{*} \gamma\right)^{m} \mid k, l \in \mathbf{Z}, m \in \mathbf{N}\right\}$ forms a linear basis for $\mathcal{A}_{q}$. So if $\mathcal{A}_{q}\left(\gamma^{*} \gamma\right)$ denotes the unital ${ }^{*}$-subalgebra of $\mathcal{A}_{q}$ generated by $\gamma^{*} \gamma$, we see that $\mathcal{A}_{q}$ is the direct sum $\bigoplus_{u, v \in \mathbf{Z}} a(u, v) \mathcal{A}_{q}\left(\gamma^{*} \gamma\right)$.

Lemma 8.7. Consider $M \in \mathbf{N}_{0}$ and $m \in \frac{1}{2} \mathbf{Z}$ so that $M=2 m+1$. If $j, k \in$ $\{-m, \ldots, m\}$, then $W_{j k}^{M} \in a(j+k, k-j) \mathcal{A}_{q}\left(\gamma^{*} \gamma\right)$.

Proof. We proceed by induction on $M$. Since $W_{00}^{1}=1$, the lemma is certainly true for $M=1$. Next we suppose that $M>1$ and that the lemma is true for $M-1$.

(1) Let $j \in\{-m, \ldots, m\}$. Clearly, $x_{-m}^{m}=\left(\gamma^{*}\right)^{2 m}$ thus $\sum_{i=-m}^{m} x_{i}^{m} \otimes W_{i,-m}^{M}=$ $\Delta\left(\gamma^{*}\right)^{2 m}=\left(\alpha \otimes \gamma^{*}+\gamma^{*} \otimes \alpha^{*}\right)^{2 m}$. Since $\alpha \otimes \gamma^{*}$ and $\gamma^{*} \otimes \alpha^{*}$ commute up to a scalar, there exist complex numbers $r_{-m}, \ldots, r_{m}$ such that

$$
\begin{aligned}
\left(\gamma^{*} \otimes \alpha^{*}+\alpha \otimes \gamma^{*}\right)^{2 m} & =\sum_{i=-m}^{m} r_{i}\left(\alpha \otimes \gamma^{*}\right)^{i+m}\left(\gamma^{*} \otimes \alpha^{*}\right)^{m-i} \\
& =\sum_{i=-m}^{m} r_{i} q^{(m+i)(m-i)} x_{i}^{m} \otimes a(i-m,-i-m) ;
\end{aligned}
$$

thus, $W_{j,-m}^{M}=r_{j} q^{(m+j)(m-j)} a(j-m,-j-m)$. 
(2) Let $k \in\{-m+1, \ldots, m\}$ and $j \in\{-m, \ldots, m\}$. Clearly, $\alpha x_{k-\frac{1}{2}}^{m-\frac{1}{2}}=x_{k}^{m}$. Hence

$$
\begin{aligned}
& \sum_{i=-m}^{m} x_{i}^{m} \otimes W_{i k}^{M}=\Delta\left(x_{k}^{m}\right)=\Delta\left(\alpha x_{k-\frac{1}{2}}^{m-\frac{1}{2}}\right)=\Delta(\alpha) \Delta\left(x_{k-\frac{1}{2}}^{m-\frac{1}{2}}\right) \\
& =\left(\alpha \otimes \alpha-q \gamma^{*} \otimes \gamma\right) \sum_{i=-m+\frac{1}{2}}^{m-\frac{1}{2}} x_{i}^{m-\frac{1}{2}} \otimes W_{i, k-\frac{1}{2}}^{M-1} \\
& \quad=\sum_{i=-m+\frac{1}{2}}^{m-\frac{1}{2}} x_{i+\frac{1}{2}}^{m} \otimes \alpha W_{i, k-\frac{1}{2}}^{M-1}-\sum_{i=-m+\frac{1}{2}}^{m-\frac{1}{2}} q^{-m-i+\frac{3}{2}} x_{i-\frac{1}{2}}^{m} \otimes \gamma W_{i, k-\frac{1}{2}}^{M-1} \\
& \quad=\sum_{i=-m+1}^{m} x_{i}^{m} \otimes \alpha W_{i-\frac{1}{2}, k-\frac{1}{2}}^{M-1}-\sum_{i=-m}^{m-1} q^{-m-i+1} x_{i}^{m} \otimes \gamma W_{i+\frac{1}{2}, k-\frac{1}{2}}^{M-1} .
\end{aligned}
$$

This implies the existence of complex numbers $c, d$ such that $W_{j k}^{M}$ is equal to $c \alpha W_{j-\frac{1}{2}, k-\frac{1}{2}}^{M-1}+d \gamma W_{j+\frac{1}{2}, k-\frac{1}{2}}^{M-1}$. Therefore the induction hypothesis implies that

$$
W_{j k}^{M} \in \alpha a(j+k-1, k-j) \mathcal{A}_{q}\left(\gamma^{*} \gamma\right)+\gamma a(j+k, k-j-1) \mathcal{A}_{q}\left(\gamma^{*} \gamma\right) .
$$

Since $\alpha a(p, q) \in a(p+1, q) \mathcal{A}_{q}\left(\gamma^{*} \gamma\right)$ and $\gamma a(p, q) \in a(p, q+1) \mathcal{A}_{q}\left(\gamma^{*} \gamma\right)$, it follows that $W_{j k}^{M} \in a(j+k, k-j) \mathcal{A}_{q}\left(\gamma^{*} \gamma\right)$.

So we have proven that $W_{j k}^{M} \in a(j+k, k-j) \mathcal{A}_{q}\left(\gamma^{*} \gamma\right)$ for all possible values of $j$ and $k$.

Lemma 8.6 will be an immediate consequence of the next one.

Lemma 8.8. Consider $M \in \mathbf{N}_{0}$ and $m \in \frac{1}{2} \mathbf{Z}$ so that $M=2 m+1$. If $j, k \in$ $\{-m, \ldots, m\}$, then $\alpha W_{j k}^{M} \in \mathbf{C} W_{j+\frac{1}{2}, k+\frac{1}{2}}^{M-1}+\mathbf{C} W_{j+\frac{1}{2}, k+\frac{1}{2}}^{M+1}$ and $\gamma W_{j k}^{M} \in \mathbf{C} W_{j-\frac{1}{2}, k+\frac{1}{2}}^{M-1}$ $+\mathbf{C} W_{j-\frac{1}{2}, k+\frac{1}{2}}^{M+1}$.

Proof. One easily checks that $W^{2}=\left(\begin{array}{c}\alpha^{*}-q \gamma \\ \gamma^{*} \\ \alpha\end{array}\right)$. Since $W_{00}^{1}=1$, this implies that the lemma is certainly true if $M=1$. From now on, we suppose that $M>1$.

By [13. Theorem 5.11], we know that the tensor product representation $W^{2} \otimes W^{M}$ is equivalent to $W^{M-1} \oplus W^{M+1}$, that is, there exists an invertible matrix $Q \in$ $M_{2 M}(\mathbf{C})$ such that $W^{2} \otimes W^{M}=Q\left(W^{M-1} \oplus W^{M+1}\right) Q^{-1}$. But $\alpha W_{j k}^{M}$ appears as a matrix element of $W^{2} \otimes W^{M}$. It follows that $\alpha W_{j k}^{M}$ belongs to $\left\langle W_{r s}^{M-1}\right| r, s=$ $\left.-m+\frac{1}{2}, \ldots, m-\frac{1}{2}\right\rangle+\left\langle W_{r s}^{M+1} \mid r, s=-m-\frac{1}{2}, \ldots, m+\frac{1}{2}\right\rangle$.

By the previous lemma, $\alpha W_{j k}^{M} \in a(j+k+1, k-j) \mathcal{A}_{q}\left(\gamma^{*} \gamma\right)$. On the other hand, we know that $W_{r s}^{M-1} \in a(r+s, s-r) \mathcal{A}_{q}\left(\gamma^{*} \gamma\right)$ and $W_{r^{\prime} s^{\prime}}^{M+1} \in a\left(r^{\prime}+s^{\prime}, s^{\prime}-r^{\prime}\right) \mathcal{A}_{q}\left(\gamma^{*} \gamma\right)$, for all $r, s \in\left\{-m+\frac{1}{2}, \ldots, m-\frac{1}{2}\right\}$ and $r^{\prime}, s^{\prime} \in\left\{-m-\frac{1}{2}, \ldots, m+\frac{1}{2}\right\}$. Note also that $j+k+1=r+s$ and $k-j=s-r \Leftrightarrow r=j+\frac{1}{2}$ and $s=k+\frac{1}{2}$. Since $\mathcal{A}_{q}$ is the direct sum $\bigoplus_{u, v} a(u, v) \mathcal{A}_{q}\left(\gamma^{*} \gamma\right)$, this implies that $\alpha W_{j k}^{M} \in \mathbf{C} W_{j+\frac{1}{2}, k+\frac{1}{2}}^{M-1}+\mathbf{C} W_{j+\frac{1}{2}, k+\frac{1}{2}}^{M+1}$. The statement concerning $\gamma$ is proven in a similar way.

We used the next result earlier in this section but prove it here because it is an easy consequence of Lemma 8.7 
Lemma 8.9. Consider $M \in \mathbf{N}_{0}$ and $m \in \frac{1}{2} \mathbf{Z}$ so that $M=2 m+1$. If $p, p^{\prime}, j, k \in$ $\{-m, \ldots, m\}$ and $(p, j) \neq\left(p^{\prime}, k\right)$, then $h\left(\left(W_{p j}^{M}\right)^{*} W_{p^{\prime} k}^{M}\right)=0$.

Proof. If $i, j, i^{\prime}, j^{\prime} \in \mathbf{Z}$ and $b, c \in \mathcal{A}_{q}\left(\gamma^{*} \gamma\right)$, [14, A 1.8] implies that

$$
h\left(\left(a\left(i^{\prime}, j^{\prime}\right) c\right)^{*}(a(i, j) b)\right)=0
$$

if $(i, j) \neq\left(i^{\prime}, j^{\prime}\right)$. By assumption, $(p+j, j-p) \neq\left(p^{\prime}+k, k-p^{\prime}\right)$. As a consequence, Lemma 8.7 implies that $h\left(\left(W_{p j}^{M}\right)^{*} W_{p^{\prime} k}^{M}\right)=0$.

It is very unlikely that the Dirac operator considered here fits into the framework of Connes' non-commutative geometry (although we do not have a proof of this fact). This provides another indication that generalisations of this non-commutative geometry have to be studied. One such generalisation is considered in [6], but we could not prove that our Dirac operator fits into this more general framework for non-commutative geometry. The problem lies in the fact that we can only prove Theorem 8.5 under the assumption that $\beta+\delta=1$, whereas the theory in [6] relies on the fact that this same theorem is true if $\beta+\delta<1$ (see [6. Section V.5]).

In the next part we collect some concrete formulas for the one-parameter groups and twist automorphism. Let $\rho$ be the modular group and let $\tau$ denote the scaling group of $\mathcal{A}_{q}$. It follows from [14, Appendix A1] that $\rho_{z}(\alpha)=q^{-2 i z} \alpha, \rho_{z}(\gamma)=\gamma$, $\tau_{z}(\alpha)=\alpha$ and $\tau_{z}(\gamma)=q^{2 i z} \gamma$, for all $z \in \mathbf{C}$.

We denote the twist automorphism of $\int$ by $\sigma$. We know from [13, Table 1] that $\theta \alpha=q^{-4} \alpha, \theta \gamma=q^{-4} \gamma$. Thus $\sigma(\alpha)=q^{-4} \rho_{i}(\alpha)=q^{-2} \alpha$. Similarly, $\sigma\left(\alpha^{*}\right)=q^{2} \alpha^{*}$, $\sigma(\gamma)=q^{-4} \gamma$ and $\sigma\left(\gamma^{*}\right)=q^{4} \gamma^{*}$. Since $\sigma$ commutes with the differential $d$, it follows from [13, Table 3] that $\sigma\left(\eta_{1}\right)=q^{6} \eta_{1}, \sigma\left(\eta_{2}\right)=\eta_{2}$ and $\sigma\left(\eta_{3}\right)=q^{-6} \eta_{3}$. In Section 7 it turned out that it is beneficial to require the inner product on $\Omega^{\text {inv }}$ to be chosen in such a way that $\sigma$ is a positive operator. Since $\eta_{1}, \eta_{2}$ and $\eta_{3}$ are eigenvectors of $\sigma$ with different eigenvalues, this requirement implies that $\eta_{1}, \eta_{2}, \eta_{3}$ are necessarily orthogonal with respect to such an inner product (which is true for the inner product used in this section). Similar remarks apply to the second order forms.

By the universality of $\mathcal{A}_{q}$ there exists a unique algebra homomorphism $\phi: \mathcal{A}_{q} \rightarrow$ $\mathcal{A}_{q}$ such that $\phi(\alpha)=q^{-1} \alpha, \phi\left(\alpha^{*}\right)=q \alpha^{*}, \phi(\gamma)=q^{-2} \gamma$ and $\phi\left(\gamma^{*}\right)=q^{2} \gamma^{*}$. One easily checks that $\phi^{2}(a)=\sigma(a), \phi\left(\phi\left(a^{*}\right)^{*}\right)=a$ and $\left(\tau_{\frac{i}{2}} \otimes \phi\right) \Delta(a)=\Delta(\phi(a))$, for all $a \in \mathcal{A}_{q}$ (it is enough to check these equalities on the generators of $\mathcal{A}_{q}$ ). Thus it follows from Corollary 7.6 that $h\left(a^{*} \sigma(a)\right) \geq 0$. By Theorem 7.5 there exists a unique exponential one-parameter group $\beta$ on $\Omega$ such that $\beta_{i}=\sigma$.

\section{Appendix: One-PARAMEter GRoups}

In this Appendix we present a self-contained brief account of the material on exponential one-parameter groups of automorphisms needed for this paper. We have included this since the material is not standard and we know of no suitable reference for it in the literature.

Let $\beta$ be a map from $\mathbf{C}$ into the set of algebra automorphisms on a $*$-algebra $B$. Suppose that $\beta_{z}(b)^{*}=\beta_{\bar{z}}\left(b^{*}\right)$ and $\beta_{y+z}=\beta_{y} \beta_{z}$, for all $b \in B$ and $y, z \in \mathbf{C}$. Then $\beta$ is called a one-parameter group on $B$.

Note that $\beta_{0}=\operatorname{id}_{B}$ and $\left(\beta_{z}\right)^{-1}=\beta_{-z}$, for $z \in \mathbf{C}$. 
One-parameter groups become even more interesting if extra analyticity conditions are imposed. In the case of compact quantum groups an exponential growth condition can also be imposed.

We say that an analytic function $f: \mathbf{C} \rightarrow \mathbf{C}$ is of exponential type if there exist positive numbers $M$ and $r$ such that $|f(z)| \leq M e^{r|\operatorname{Im} z|}$, for all $z \in \mathbf{C}$.

It is easy to check that the functions of exponential type form an algebra. It is equally clear that for any positive number $\lambda$, the function $\mathbf{C} \rightarrow \mathbf{C}: z \mapsto \lambda^{i z}$ is of exponential type. The translate of a function of exponential type is another such function.

Consider a vector space $V$ and a function $f: \mathbf{C} \rightarrow V$. We say that $f$ is of finite exponential type if there exist elements $v_{1}, \ldots, v_{n} \in V$ and functions $f_{1}, \ldots, f_{n}$ of exponential type such that $f(z)=\sum_{j=1}^{n} f_{j}(z) v_{j}$, for all $z \in \mathbf{C}$.

Let $f, g: \mathbf{C} \rightarrow V$ be two functions of finite exponential type and let $c$ be a non-zero number on the imaginary axis. Then, by [14 Lemma 5.5], $f=g$ if and only if $f(n c)=g(n c)$, for all $n \in \mathbf{N}_{0}$.

Consider a one-parameter group $\beta$ on $\mathrm{a}^{*}$-algebra $B$ such that for every $b \in B$, the function $\mathbf{C} \rightarrow B: z \mapsto \beta_{z}(b)$ is of finite exponential type. Then we call $\beta$ an exponential one-parameter group on $B$.

Let $c$ be a non-zero number on the imaginary axis. Then an exponential oneparameter group on $B$ is completely determined by its value at $c$ : If $\beta$ and $\gamma$ are exponential one-parameter groups on $B$, then $\beta=\gamma$ if and only if $\beta_{c}=\gamma_{c}$.

Another basic fact is the following: If $\tau$ is a linear functional on $B$ such that $\tau \beta_{c}=\tau$, then $\tau \beta_{z}=\tau$, for all $z \in \mathbf{C}$. This is true because, for every $b \in B$, the function $\mathbf{C} \rightarrow \mathbf{C}: z \mapsto \tau\left(\beta_{z}(b)\right)$ is of exponential type and $\tau\left(\beta_{n c}(b)\right)=\tau(b)$, for all $n \in \mathbf{N}_{0}$.

In order to check that an algebra automorphism on a *-algebra is induced by an exponential one-parameter group, it is enough to check it on a set of generators:

Theorem 9.1. Let $B$ be $a^{*}$-algebra and $S$ a subset such that $S \cup\{1\}$ generates $B$ as a *-algebra. Let $\phi$ be an algebra homomorphism on $B$ satisfying $\phi\left(\phi\left(b^{*}\right)^{*}\right)=b$, for all $b \in B$. Assume for every $b \in S$ the existence of a function $f: \mathbf{C} \rightarrow B$ of finite exponential type such that $f(n i)=\phi^{n}(b)$, for all $n \in \mathbf{N}$. Then there exists a unique exponential one-parameter group $\beta$ on $B$ such that $\beta_{i}=\phi$.

Proof. Define $T$ to be the set of elements $b \in B$ such that there exist a function $f: \mathbf{C} \rightarrow B$ of finite exponential type satisfying $f(n i)=\phi^{n}(b)$, for all $n \in \mathbf{N}$. Note that $f$ is unique; we denote it by $f_{b}$. Clearly, $1 \in T$ and $f_{1}(z)=1$, for all $z \in \mathbf{C}$. By assumption $S \subseteq T$.

The set $T$ is self-adjoint: To see this, let $b \in T$ and $m \in \mathbf{N}_{0}$. Then the functions $\mathbf{C} \rightarrow B: z \mapsto \phi^{m}\left(f_{b}(z)\right)$ and $\mathbf{C} \rightarrow B: z \mapsto f_{b}(z+m i)$ are of finite exponential type and agree on the set $\mathbf{N}_{0} i$. So they must be equal on the whole complex plane. Hence, $\phi^{m}\left(f_{b}(-m i)\right)=f_{b}(0)=b$. Therefore, $f_{b}(-m i)=\phi^{-m}(b)$ and $f_{b}(-m i)^{*}=\phi^{m}\left(b^{*}\right)$. It follows that $b^{*} \in T$ and $f_{b^{*}}(z)=f_{b}(\bar{z})^{*}$, for all $z \in \mathbf{C}$.

If $b, b^{\prime} \in T$ and $c, d \in \mathbf{C}$, linearity of each $\phi^{n}$ implies easily that $c b+d b^{\prime} \in T$ and $f_{c b+d b^{\prime}}=c f_{b}+d f_{b^{\prime}}$. Also, multiplicativity of each $\phi^{n}$ implies that $b b^{\prime} \in T$ and $f_{b b^{\prime}}=f_{b} f_{b^{\prime}}$. It follows that $T$ is a ${ }^{*}$-subalgebra of $B$ containing $S$ and 1 , and therefore it is equal to $B$.

Define the function $\beta$ from $\mathbf{C}$ into the set of mappings on $B$ by setting $\beta_{z}(b)=$ $f_{b}(z)$, for all $z \in \mathbf{C}$ and $b \in B$. The preceding considerations imply, for every $z \in \mathbf{C}$, 
that $\beta_{z}$ is an algebra homomorphism and that $\beta_{z}(b)^{*}=\beta_{\bar{z}}\left(b^{*}\right)$, for all $b \in B$. It is also clear that $\beta_{n i}=\phi^{n}$, for all $n \in \mathbf{N}_{0}$.

Let $b \in B, y \in \mathbf{C}$, and $m, n \in \mathbf{N}_{0}$. The functions $\mathbf{C} \rightarrow B: z \mapsto \beta_{z}\left(\beta_{m i}(b)\right)$ and $\mathbf{C} \rightarrow B: z \mapsto \beta_{z+m i}(b)$ are of finite exponential type and $\beta_{n i}\left(\beta_{m i}(b)\right)=$ $\phi^{n}\left(\phi^{m}(b)\right)=\phi^{n+m}(b)=\beta_{n i+m i}(b)$. Hence, both functions agree on the whole complex plane; in particular $\beta_{y}\left(\beta_{m i}(b)\right)=\beta_{y+m i}(b)$.

Since the functions $\mathbf{C} \rightarrow B: z \mapsto \beta_{y}\left(\beta_{z}(b)\right)$ and $\mathbf{C} \rightarrow B: z \mapsto \beta_{y+z}(b)$ are both of finite exponential type, the preceding considerations imply that both functions agree on the whole complex plane; hence, $\beta_{y}\left(\beta_{z}(b)\right)=\beta_{y+z}(b)$, for all $y, z \in \mathbf{C}$. From all this we conclude that $\beta$ is an exponential one-parameter group on $B$.

Lemma 9.2. Let $B$ be $a^{*}$-algebra, let $\pi: B \rightarrow B$ be a linear map and let $\beta$ be an exponential one-parameter group on $B$. Let $c$ be a non-zero number on the imaginary axis. If $\pi \beta_{c}=\beta_{c} \pi$ (respectively, $\pi \beta_{c}=\beta_{-c} \pi$ ), then $\pi \beta_{z}=\beta_{z} \pi$ (respectively, $\left.\pi \beta_{z}=\beta_{-z} \pi\right)$, for all $z \in \mathbf{C}$.

Proof. We prove the result only in the first case, where $\pi \beta_{c}=\beta_{c} \pi$; the proof of the other case is almost the same. Let $b \in B$. It is clear that the functions $\mathbf{C} \rightarrow B: z \mapsto \pi\left(\beta_{z}(b)\right)$ and $\mathbf{C} \rightarrow B: z \mapsto \beta_{z}(\pi(b))$ are of finite exponential type. Our assumption implies that $\pi \beta_{n c}=\beta_{n c} \pi$, for all $n \in \mathbf{N}_{0}$, implying that the above functions are equal on $\mathbf{N}_{0} i$, and therefore on the whole complex plane.

\section{REFERENCES}

[1] E. ABE, Hopf Algebras, Cambridge University Press, Cambridge (1980). MR 0594432 (83a:16010)

[2] A. Connes, Noncommutative Geometry, Academic Press, San Diego-New York (1994). MR1303779 (95j:46063)

[3] K. Schmüdgen, Commutator representations of covariant differential calculi on quantum groups, Lett. Math. Phys. 59 (2002), 95-106. MR1894810 (2003d:58013)

[4] K. Schmüdgen, Commutator representations of differential calculi on the quantum group $S U_{q}(2)$, J. Geom. Phys. 31 (1999), 241-264. MR1711531 (2000h:58019)

[5] A.U. Klymik, K. Schmüdgen, Quantum Groups and their Representations, Springer, Heidelberg-New York (1998). MR1492989 (99f:17017)

[6] A. Jaffe, Quantum harmonic analysis and geometric invariants, Advances in Math. 143 (1999), 1-110. MR.1680658(2000f:58064)

[7] J. Kustermans, G.J. Murphy, L. Tuset, Differential calculi over quantum groups and twisted cyclic cocycles, J. Geom. Phys. 44 (2003), 570-594. MR.1943179

[8] J. Kustermans, L. Tuset, A survey of C*-algebraic quantum groups I, Irish Math. Soc. Bull. 43 (1999), 8-63. MR1741102 (2001i:46090)

[9] I. Heckenberger, Hodge and Laplace-Beltrami operators for bicovariant differential calculi on quantum groups, Compositio Math. 123 (2000), 329-354. MR1795294 (2001k:58011)

[10] I. Heckenberger, A. Schüler, De Rham Cohomology and Hodge decomposition for Quantum Groups, Proc. London Math. Soc. (3) 83 (2001), 743-768. MR1851089 (2003b:58008)

[11] G.J. Murphy, L. Tuset, Compact quantum groups, preprint, National University of Ireland, Cork (1999).

[12] A. Van Daele, An algebraic framework for group duality, Advances in Math. 140 (1998), 323-366. MR 1658585 (2000g:16045)

[13] S.L. WoronOWICZ, Twisted $S U(2)$ group - an example of a non-commutative differential calculus, Publ. RIMS Kyoto Univ. 23 (1987), 117-181. MR0890482 (88h:46130)

[14] S.L. Woronowicz, Compact matrix pseudogroups, Comm. Math. Phys. 111 (1987), 613665. MR0901157 (88m:46079) 
[15] S.L. Woronowicz, Compact quantum groups, Symétries quantiques (Les Houches) (1998), 845-884, North-Holland, Amsterdam. MR1616348 (99m:46164)

[16] S.L. Woronowicz, Differential calculus on compact matrix pseudogroups (quantum groups), Commun. Math. Phys. 122 (1989), 125-170. MR0994499(90g:58010)

Departement Wiskunde, KU Leuven, Celestijnenlaan 200B, 3000 Leuven, Belgium

E-mail address: j.kustermans@skynet.be

Department of Mathematics, National University of Ireland, Cork, Ireland

E-mail address: g.j.murphy@ucc.ie

Faculty of Engineering, University College, Oslo, Norway

E-mail address: Lars.Tuset@iu.hio.no 